\author{
Universidade de São Paulo \\ Instituto de Física
}

\title{
Propriedades eletrônicas de sistemas conjugados: importância da troca exata
}

\author{
José Maximiano F. Pinheiro Junior
}

Orientadora: Profa. Dra. Marília Junqueira Caldas

Tese de Doutorado apresentada ao Instituto de Física para a obtenção do título de Doutor em Ciências

Comissão examinadora:

1. Profa. Dra. Marília Junqueira Caldas (Orientadora - IFUSP)

2. Prof. Dr. Sylvio Roberto Accioly Canuto (IFUSP)

3. Prof. Dr. Koiti Araki (IQUSP)

4. Prof. Dr. Vitor Rafael Coluci (UNICAMP)

5. Prof. Dr. Paulo Barbeitas Miranda (IFSC-USP)

São Paulo

2014 


\section{FICHA CATALOGRÁFICA}

Preparada pelo Serviço de Biblioteca e Informação

do Instituto de Física da Universidade de São Paulo

Pinheiro Junior, José Maximiano Fernandes

Propriedades eletrônicas de sistemas conjugados:

importância da troca exata. - São Paulo, 2014

Tese (Doutorado) - Universidade de São Paulo.

Instituto de Física. Depto. de Física dos Materiais e Mecânica.

Orientadora: Profa. Dra. Marília Junqueira Caldas Área de Concentração: Física

Unitermos: 1. Física da matéria condensada; 2. Física teórica; 3. Física computacional.

USP/IF/SBI-043/2014 
Dedico esta Tese com amor e gratidão aos meus queridos pais, José e Rosário, e minha irmã, Arianne, pilares a partir do qual me ergui como homem de bem e hoje cientista, $e$ que sempre me proporcionaram uma base sólida para que o sonho deste e de outros trabalhos seja possivel. 


\section{AGRADECIMENTOS}

A Tese de Doutoramento apresentada nas páginas a seguir foi fruto de muita dedicação e intenso trabalho de pesquisa, mas certamente não teria se concretizado não fosse a inestimável contribuição e apoio de muitas pessoas, amigos e familiares, e também das várias instituições de fomento. Assim, esta seção é reservada para dar o devido reconhecimento e meus mais sinceros agradecimentos a todos que, direta ou indiretamente contribuíram para o sucesso deste trabalho.

Primeiramente, devo agradecer aos colegas do grupo Nanomol Regina, Francisco, Járleson, Rodrigo e Leonardo que não apenas deram contribuições muito importantes para superar os diversos problemas técnicos e científicos do trabalho, mas que também sempre contagiavam a todos com muito entusiasmo, otimismo e alegria em prol de um objetivo comum, tornando o ambiente de trabalho mais produtivo.

Entre os diversos amigos que tive o prazer de encontrar em São Paulo, não posso deixar de agradecer ao Amaury, Evandro, Petersen, Ricardo e a Magnólia pelos vários momentos de diversão e descontração extremamente necessários para manter o bom humor, e também pelo companheirismo e palavras de conforto e estímulo tão importantes para não sucumbir as dificuldades.

À secretaria do grupo Nanomol Sandra Regina devo meus sinceros agradecimentos pelas inúmeras vezes que se dispôs a solucionar com muita cordialidade os mais diversos problemas burocráticos que certamente teriam tomado um precioso tempo de dedicação à pesquisa.

Aos técnicos Sérgio e Tales do grupo teórico Nanomol vai o meu grande apreço pela ajuda constante na solução dos meus problemas computacionais.

Esta Tese contou ainda com a valiosa contribuição de colaboradores do Instituto FritzHaber (FHI) de Berlim - Al. Além do suporte computacional gentilmente concedido pelo FHI, que elevaram os cálculos desta Tese a um nível de excelência e competitividade mundial, contamos também com idéias e sugestões enriquecedores por parte de nossos colaboradores do FHI, aos quais sou profundamente grato. Em particular, agradeço aos doutores Volker Blum e Patrick Rinke, pesquisadores excepcionais que tiveram paciência em me orientar durante minha estadia no FHI, e cuja os ensinamentos marcaram profundamente minha 
formação acadêmica. A todos os demais estudantes e funcionários do FHI que sempre me acolheram com muita cordialidade tornando o ambiente de trabalho em terras desconhecidas muito mais tranquilo, deixo aqui um sincero Danke Schön.

Meu reconhecimento vai também aos diversos orgão de fomento - CNPq, FAPESP e INEO - que viabilizaram e acreditaram em um projeto tão ambicioso concedendo todo o apoio técnico-financeiro necessário para o desenvolvimento deste trabalho. Agradeço, em especial, a CAPES por me conceder a bolsa de doutorado.

À professora Marília Caldas deixo aqui meus sinceros agradecimentos pela paciência e dedicação com que me orientou ao longo deste trabalho, e pela invejável competência em identificar falhas e sabiamente sugerir novos caminhos a seguir. Muito obrigado pela oportunidade e por acreditar na minha capacidade para realizar um projeto tão audacioso!

Em especial, agradeço à minha querida família pelo apoio incondicional, e que mesmo distantes estiveram sempre presente nos momentos mais difíceis me encorajando a continuar a jornada do doutoramento, superando todos os percalços no caminho. Presto-lhes grande admiração, gratidão e amor eternos.

Diante da extensa lista de nomes e ao espaço limitado desta seção, seria impossível citar todas as pessoas as quais eu gostaria de expressar minha profunda gratidão. Desta forma, peço a todos que contribuiram de alguma forma para este trabalho que sintam-se devidamente representados em cada frase desta Tese no qual a primeira pessoal do plural for empregada.

(José Maximiano F. Pinheiro Júnior) 

Eres libre de tomar las opciones que quieras, pero eres prisionero de sus consecuencias.

(Pablo Neruda) 



\section{Resumo}

Polímeros conjugados semicondutores tem atraído grande interesse nas últimas décadas devido às possíveis aplicações como componentes ativos em aplicações optoeletrônicas. A adequação destes semicondutores orgânicos para a fabricação de dispositivos depende do entendimento e controle de propriedades eletrônicas básicas: gap fundamental $\left(\mathrm{E}_{g}\right)$ e potencial de ionização (IP). Nesse contexto, estudos teóricos baseados em cálculos de primeiros princípios tem se mostrado muito úteis, uma vez que possibilitam a simulação de processos físicos em condições ideais, onde se pode analisar as propriedades eletrônicas de polímeros desconsiderando efeitos do ambiente ou desordem estrutural. A Teoria do Funcional da Densidade (DFT) tem se tornado o método mais comum para o cálculo da estrutura eletrônica do estado fundamental de uma ampla variedade de materiais orgânicos complexos. Embora cálculos DFT baseados na diferença de energias totais tem sido aplicados com sucesso para estimar IPs de moléculas pequenas, este método falha nas propriedades de sistemas conjugados longos. Realmente, a capacidade preditiva da DFT padrão com respeito as propriedades espectroscópicas é frequentemente limitada, entretanto o tratamento adequado das excitações eletrônicas através de abordagens de muitos corpos é ainda muito difícil para materiais orgânicos complexos. Funcionais híbridos que misturam uma fração $(\alpha)$ de troca exata (EX) não-local ao correspondente semi-local representam uma boa alternativa, embora a quantidade ideal de EX seja, em geral, dependente do sistema.

Neste trabalho, adotamos um esquema não-empírico baseado na aproximação $\mathrm{G}_{0} \mathrm{~W}_{0}$ para identificar o valor ótimo de $\alpha$ para o funcional híbrido PBE no qual a correção de autoenergia para o orbital mais alto ocupado (HOMO) de Kohn-Sham generalisado é minimizado. Estudamos, com base nessa estratégia, a dependência com o comprimento das propriedades eletrônicas básicas em uma família de oligômeros conjugados 1D de trans-poliacetileno (TPA). Nossos cálculos mostram que a fração EX ótima (dependente do tamanho) incorporada ao PBEh reproduz com precisão os IPs experimentais determinados em fase gasosa, 
embora nenhum vínculo adicional tenha sido imposto a priori. Além disso, notamos que o valor ótimo de $\alpha$ decresce exponencialmente com o comprimento da cadeia, variando de $\alpha \bumpeq 0,85$ para o menor oligômero (etileno, $\mathrm{n}=1$ ) até $\alpha \bumpeq 0.75$ extrapolado para a cadeia de TPA isolada. A precisão do nosso PBEh otimizado para prever IPs e $\mathrm{E}_{g}$ é superior a outras abordagens de campo médios convencionais, como demonstrado para um conjunto selecionado de moléculas conjugadas, tais como acenes e fenilenos. Como resultado, podemos obter boas estimativas para as barreiras de energia de transferência de elétrons em interfaces de orgânicos/orgânicos.

No outro extremo, analisamos a influência da troca exata sobre a estrutura eletrônica do sistema de metal prototípico ouro $(\mathrm{Au})$, comumente usado como eletrodo em dispositivos orgânicos. Neste caso, podemos confirmar o resultado esperado que a inserção de mesmo uma pequena fração do EX no funcional PBE distorce a estrutura de bandas do Au, piorando a descrição de propriedades eletrônicas em relação ao PBE regular.

Em seguida, prosseguimos com a análise da viabilidade de estudar sistemas de interface polímero/metal utilizando o formalismo DFT padrão. Nossos cálculos mostram que o resultado é muito dependente do sistema: para a interface TPA/Au(111), uma transferência de carga artificial ocorre na interface devido à subestimativa dos IPs do sistema conjugado inerente à aproximação DFT subjacente.

Finalmente, o nosso estudo enfatiza a importância de uma escolha fisicamente motivada de fração EX em funcionais híbridos para prever com precisão tanto os potenciais de ionização quanto os gaps elétricos de semicondutores orgânicos relevantes para a nanoeletrônica. 


\section{Abstract}

Semiconducting conjugated polymers have attracted considerable interest over the past decades due to the promising applications as active components for optoelectronic applications. The suitability of such organic semiconductors for device fabrication relies on quantitative understanding and control of basic electronic properties: fundamental gap $\left(\mathrm{E}_{g}\right)$ and ionization potential (IP). In this context, theoretical studies based on first principles approaches have proven useful, through simulating physical processes in ideal conditions, in which one might analyse the electronic properties of polymers apart from the effects of the surrounding environment or structural disorder. Density Functional Theory (DFT) has become an usual choice for calculating the ground state electronic structure of a wide variety of complex organic materials. Although DFT calculations based on total energy differences have been successfully applied to estimate IPs of small molecules, they fail for properties of long conjugated systems. Indeed, the predictive ability of standard DFT with respect to spectroscopic properties is often limited, however a proper treatment of the electronic excitations through many-body approaches is still very difficult for complex organic materials. Hybrid functionals that mix a fraction $(\alpha)$ of nonlocal exact exchange (EX) with the semilocal counterpart represent a good alternative, although the ideal amount of EX is usually system dependent.

In this work, we adopt a non-empirical scheme based on the $\mathrm{G}_{0} \mathrm{~W}_{0}$ approximation to identify the optimum $\alpha$ value for the PBE hybrid functional for which the self-energy correction to the generalized Kohn-Sham highest occupied molecular orbital (HOMO) is minimized. Based on this strategy we study the size dependence of the basic electronic properties in a family of 1D $\pi$-conjugated oligomers of trans-polyacetylene (TPA). Our calculations demonstrate that the size dependent optimal EX fraction incorporated in PBEh accurately reproduces IPs from experimental gas phase data, although no particular constraint has been imposed a priori. Furthermore, we note that the optimum $\alpha$-value decreases exponen- 
tially with chain length going from $\alpha \bumpeq 0.85$ for the smaller oligomer (ethylene, $\mathrm{n}=1$ ) up to $\alpha \bumpeq 0.75$ extrapolated for an isolated TPA chain. The accuracy of our optimized PBEh in predicting IPs and $\mathrm{E}_{g}$ is superior to other conventional mean field approaches, as demonstrated for a selected set of conjugated molecules such as acenes and phenylenes. As a result, we can obtain good estimations for the energy barriers of electron transfer in organic/organic interfaces.

On the other extreme, we analyse the influence of exact exchange on the electronic structure of the prototypical metal system gold $(\mathrm{Au})$, commonly used as electrode in organic devices. In this case, we confirm the expected result that the insertion of even a small fraction of EX into PBE functional distorts the $\mathrm{Au}$ band structure, worsening the description of electronic properties compared to regular PBE.

We then proceed to analyse the factibility of studying polymer/metal interface systems using pure DFT. Our calculations reveal that the result is too system-dependent: for the $\mathrm{TPA} / \mathrm{Au}(111)$ interface, an artificial charge transfer takes place at interface due to an underestimation of the IPs of the conjugated system inherent to the underlying DFT approximation.

Finally, our study emphasizes the importance of a physically motivated choice of EX fraction in hybrid functionals for accurately predicting both ionization potentials and fundamental gaps of organic semiconductors relevant for nanoelectronics. 


\section{Sumário}

Resumo ix

Abstract

\begin{tabular}{lll}
\hline 1 & Introdução & 1
\end{tabular}

2 Modelos Teóricos para Cálculos de Estrutura Eletrônica 5

2.1 O Método Hartree-Fock . . . . . . . . . . . . . . . . . . . . . . 6

2.1 .1 Energia de Ionização: $\triangle \mathrm{SCF}$ e Teorema de Koopmans. . . . . . . . . 9

2.2 Teoria do Funcional da Densidade . . . . . . . . . . . . . . . . . . . . . . . . 12

2.3 Teoria de perturbação muitos-corpos: o método GW $\ldots$. . . . . . . . . . . . 18

3 Estrutura Eletrônica do Sistema Conjugado Modelo: Poliacetileno 21

3.1 Resultados de campo médio padrão: DFT vs. Hartree-Fock . . . . . . . . . . . 22

3.2 Otimizando funcionais híbridos para sistemas conjugados . . . . . . . . . . . 27

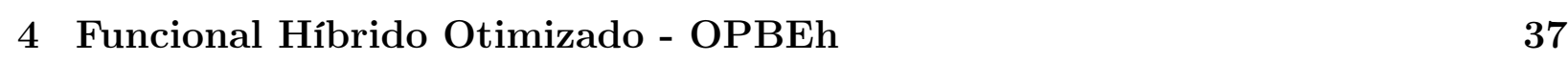

4.1 Sistemas Conjugados 1D . . . . . . . . . . . . . . . . . . . . 37

4.2 Outras Moléculas Orgânicas Conjugadas . . . . . . . . . . . . . . . . . . . . 41

4.3 Moléculas Orgânicas Alquiladas . . . . . . . . . . . . . . . . . . . . . 48

4.4 Aplicação do OPBEh a Interfaces Orgânicas . . . . . . . . . . . . . . . . . . 51

5 Interfaces Polímero/Metal: Problemas da DFT $\quad 57$

$5.1 \quad$ Propriedades Estruturais do $\mathrm{Au}-\mathrm{DFT}$. . . . . . . . . . . . . . . 58

5.1 .1 Bulk . . . . . . . . . . . . . . . . . . . . . . . . 58

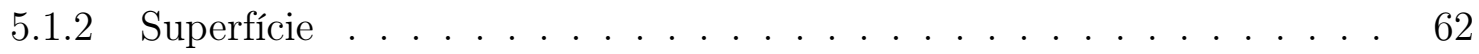


$5.2 \quad$ Propriedades eletrônicas - DFT versus DFTh $\ldots \ldots \ldots$. . . . . . . . . . 65

$5.2 .1 \quad$ Bulk . . . . . . . . . . . . . . . . . . . . . 65

5.2 .2 Superfície . . . . . . . . . . . . . . . . . . 68

$5.3 \quad$ Interfaces Polímero/Au: TPA e PE . . . . . . . . . . . . . . . 70

$5.3 .1 \quad$ Propriedades Estruturais - DFT . . . . . . . . . . . . . . . . . 71

5.3 .2 Estrutura Eletrônica: Problemas da DFT. . . . . . . . . . . . . . . 76

$\begin{array}{lll}6 & \text { Conclusão } & 83\end{array}$

\begin{tabular}{|l|l}
\hline A Interações de van der Waals & 87
\end{tabular}

\begin{tabular}{|ll}
\hline B O pacote computacional "FHI-aims" & 91
\end{tabular}

\begin{tabular}{ll}
\hline Referências & 95
\end{tabular} 


\section{Capítulo 1}

\section{Introdução}

Polímeros conjugados oferecem uma rica escolha para pesquisas em eletrônica orgânica, uma vez que são atualmente considerados um caminho para o futuro em aplicações de conversão fotovoltaica de energia, diodos emissores de luz de grande área e mesmo transistores de efeito de campo [1-4]. Aqui, o importante é a leveza e flexibilidade do dispositivo final. De outro lado, exibem características muito especiais - e complexas - de morfologia, e de estrutura eletrônica. Estes sistemas são intrinsecamente desordenados, compostos de cadeias longas em conformações enoveladas ou empacotadas, ou também de oligômeros curtos em diferentes empacotamentos ou, nestes anos recentes, de blendas incluindo uma composição específica de polímeros ou oligômeros e moléculas orgânicas derivadas do fulereno [5]. A base comum a essa classe de materiais é o comportamento semicondutor e a conjugação $\pi$ dos estados eletrônicos ativos de fronteira, para condução elétrica (principalmente de buracos) ou atividade ótica.

Em dispositivos orgânicos reais, a funcionalidade e eficiência dependem criticamente da estrutura seja morfológica seja a resultante eletrônica das múltiplas interfaces existentes. Em particular, um problema recorrente é o alinhamento dos níveis de energia [6] tanto nas interfaces orgânico/orgânico como também nas interfaces orgânico/eletrodo, em geral um metal. No primeiro caso, a diferença relativa entre o nível de energia mais alto ocupado (HOMO) do componente orgânico doador (D) e o nível mais baixo desocupado (LUMO) do aceitador (A) está diretamente relacionada as barreiras para transferência de carga do material D para o A, como representado esquematicamente no diagrama de níveis da Fig. 1.1. Analogamente para as interfaces orgânico/metal, a posição do nível de Fermi ou função 
trabalho do eletrodo metálico relativamente aos orbitais de fronteira do material orgânico determina a eficiência para o transporte de elétrons no dispositivo, como um todo.

Além de todas essas janelas de aplicação tecnológica, polímeros conjugados apresentam uma estrutura quase-unidimensional que confere propriedades de delocalização e confinamento de uma só vez, o que os torna muito interessantes para o estudo acadêmico de estrutura eletrônica. De fato, tais sistemas vem sendo estudados de longa data, através de formalismos variados desde semi-empíricos $[9,10]$ até métodos de campo médio de primeiros princípios [11], e inclusive através de teoria de perturbação de muitos corpos [12]. Na realização de dispositivos, é importante ter conhecimento das propriedades eletrônicas usuais de materiais semicondutores, como potencial de ionização e gap elétrico, dos sistemas em forma de filmes, e em contato com os eletrodos. Em contraste, o estudo teórico de sistemas tão complexos exige na prática uma modelização bastante simplificada, que nos leva ao polímero "ideal" ou seja, isolado e muitas vezes infinito periódico.

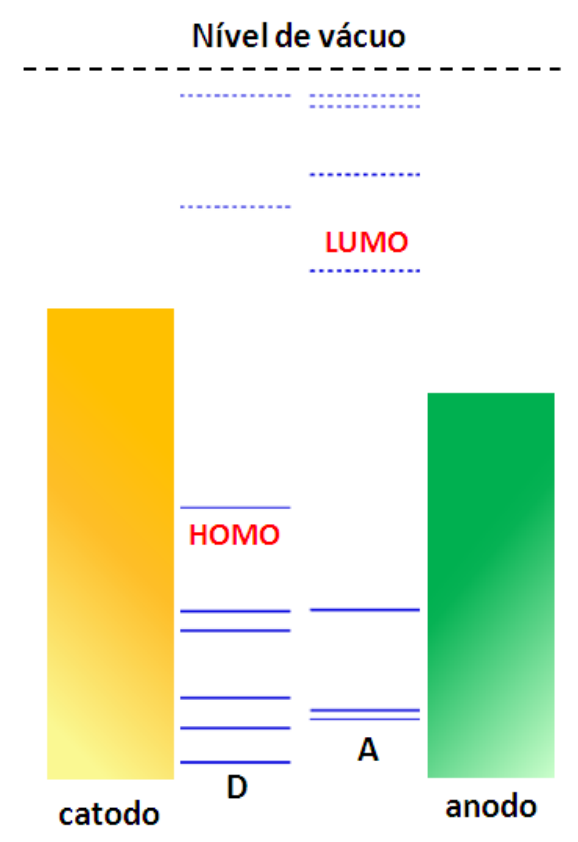

Figura 1.1: Diagrama de alinhamento de níveis de energia no limite Shottky-Mott para um dispositivo eletrônico orgânico de múltiplas camadas.

A característica unidimensional de polímeros ideais é bastante interessante para o estudo acadêmico do reflexo de diferentes formalismos de campo médio no resultado final para propriedades mensuráveis experimentalmente, como potencial de ionização e afinidade ele- 
trônica (ou o gap eletrônico), e para estimar a capacidade de previsão do método quando tais medidas não são facilmente obteníveis. A própria aplicabilidade dos métodos de campo médio, e em particular a aplicação da Teoria do Funcional da Densidade (DFT) em suas muitas variações é um campo de estudo muito atual [13. Especialmente no caso de sistemas orgânicos, métodos DFT híbridos que incluem uma fração constante de troca exata (Hartree-Fock) tem sido bastante utilizados como uma alternativa para suplantar algumas deficiências da DFT, melhorando a descrição da estrutura eletrônica desses sistemas.

Neste trabalho estudamos a aplicação de funcionais específicos ao estudo de propriedades eletrônicas do sistema conjugado modelo, trans-acetileno, e para isso exploramos diferentes formulações da DFT. Com base na metodologia adotada, estudamos a convergência de várias propriedades eletrônicas dos oligômeros conjugados em termos de seu tamanho, especialmente aquelas mais importantes para a descrição do alinhamento de níveis na interface, tais como gap elétrico e energia de ionização. Além disso, estudamos também outras moléculas orgânicas conjugadas, todas constituidas puramente de carbono e hidrogênio mas de famílias e características aromáticas diferentes, e propomos uma maneira eficiente de tratá-las dentro da DFT. Nossos resultados levam a valores de potencial de ionização e gap em muito boa concordância com os resultados disponíveis na literatura experimental, o que indica uma capacidade previsiva também muito boa para sistemas onde esses dados não estão disponíveis.

Considerando agora o problema das interfaces híbridas em uma abordagem teórica, o estudo da interface metal/polímero é intrinsecamente difícil em parte devido ao caráter muito diferente dos dois materiais, metais e filmes orgânicos, e por outro lado devido à complexidade da morfologia dos filmes poliméricos utilizados. Por exemplo com respeito a morfologia, as interfaces metal sobre material orgânico são muito diferentes das interfaces orgânico sobre metal. Além disso, os metais são materiais de gap nulo com elétrons completamente delocalizados, apresentando portanto um caráter eletrônico bastante distinto do observado em materiais orgânicos, o que dificulta o tratamento do sistema interagente orgânico/metal com um único modelo de campo médio. Experimentalmente, os detalhes dos processos físicos e químicos existentes na interface são também de difícil acesso. Assim, temos basicamente dois níveis distintos de dificuldade para a modelagem teórica: (a) obter uma morfologia inicial razoável da camada orgânica e da interface, e (b) extrair a partir de uma dada morfologia a estrutura eletrônica correta, em particular a densidade de carga correta através da interface, e o alinhamento de níveis correto. Isto requer o uso de metodologias reconhecidamente 
confiáveis. Entretanto, o processo de transferência de carga em interfaces metal-polímero permanece ainda como tema de intensos debates na literatura teórica devido a algumas questões: a transferência de carga depende essencialmente do alinhamento entre os níveis de energia dos dois materiais, alinhamento este que é reconhecidamente problemático na Teoria do Funcional Densidade (DFT), mesmo para sistemas inorgânicos.

O estudo teórico de interfaces híbridas foi realizada neste trabalho para a configuração específica de polímero sobre metal, possibilitando a modelagem de um sistema mais simples do ponto de vista estrutural, o que permite um foco mais bem definido nas propriedades eletrônicas. Para isto selecionamos como sistemas também prototípicos, trans-poliacetileno, polietileno e ouro, para os quais os problemas existentes no tratamento através de um mesmo modelo de campo médio se tornam mais evidentes, devido aos componentes orgânicos apresentarem características intrinsecamente distintas na densidade eletrônica. 


\section{Capítulo 2}

\section{Modelos Teóricos para Cálculos de Estrutura Eletrônica}

Ao longo das últimas décadas o cálculo ab initio da estrutura eletrônica de moléculas e sólidos tem se tornado uma das mais importantes ferramentas em física do estado sólido. Esses métodos nos permitem prever algumas propriedades de sistemas condensados (estrutura cristalina, geometria molecular, energias de adsorção e coesão, entre outras), sem a utilização de parâmetros empíricos; além disso, estudamos tais sistemas em condições ideais que são muito difíceis ou mesmo impossíveis de acessar experimentalmente. Podemos também obter uma visão mais clara sobre a origem de alguns fenômenos complexos que não podem ser explicados apenas com base em modelos simples ou dados experimentais. Entretanto, os métodos ab initio exigem um grande esforço computacional quando comparados aos métodos empíricos ou semi-empíricos, sendo portanto aplicáveis apenas a modelos ideais (infinitos periódicos) ou finitos mas de tamanho reduzido. De qualquer maneira, várias aproximações devem ser feitas, o que leva a questões importantes quanto à precisão e aplicabilidade desses modelos teóricos. Neste capítulo, faremos uma breve exposição dos conceitos básicos envolvidos na formulação da teoria ab initio, dedicando uma atenção especial às técnicas utilizadas nesta tese. Primeiramente, discutiremos os aspectos fundamentais dos modelos de campo médio (Hartree-Fock e DFT) que nos permitem já obter propriedades do estado fundamental para um sistema de muitas partículas com boa precisão. Em seguida, passamos à discussão de um dos métodos perturbativos que vão além da aproximação de campo médio, incluindo efeitos de muitos corpos, e permitem um tratamento adequado das 
excitações eletrônicas de uma partícula: especificamente, o método baseado na função de Green, conhecido como método GW.

\subsection{O Método Hartree-Fock}

A dinâmica dos núcleos e elétrons em sistemas poliatômicos tais como sólidos ou moléculas é completamente descrito pelo Hamiltoniano de muitos corpos dado pela equação abaixo em unidades atômicas

$$
\begin{aligned}
\mathcal{H}= & -\sum_{i=1}^{N} \frac{1}{2} \nabla_{i}^{2}-\sum_{A=1}^{M} \frac{1}{2 M_{A}} \nabla_{A}^{2} \\
& -\sum_{i=1}^{N} \sum_{A=1}^{M} \frac{Z_{A}}{\left|\mathbf{r}_{i}-\mathbf{R}_{A}\right|}+\sum_{i=1}^{N} \sum_{j>i}^{N} \frac{1}{\left|\mathbf{r}_{i}-\mathbf{r}_{j}\right|}+\sum_{A=1}^{M} \sum_{B>A}^{M} \frac{Z_{A} Z_{B}}{\left|\mathbf{R}_{A}-\mathbf{R}_{B}\right|}
\end{aligned}
$$

onde $i, j$ são os índices para os $\mathrm{N}$ elétrons e $A, B$ são os índices para os $\mathrm{M}$ núcleos. Os dois primeiros termos da equação 2.1 representam os operadores de energia cinética dos elétrons e núcleos, respectivamente, enquanto os três termos restantes estão relacionados à interação eletrostática entre as partículas. Embora a forma do Hamiltoniano para um sistema de muitas partículas seja conhecida exatamente, o problema central é encontrar o conjunto de auto-funções e auto-valores de $\mathcal{H}$ que satisfaçam à equação de Schröedinger não-relativística e independente do tempo:

$$
\mathcal{H} \Psi(\mathbf{r}, \mathbf{R})=E \Psi(\mathbf{r}, \mathbf{R})
$$

onde E é a energia total do sistema e $\Psi$ é a função de onda de muitos corpos, que depende explicitamente das posições atômicas dos núcleos e dos elétrons. Entretanto, sabe-se que já o problema de duas partículas interagentes sob a ação de um potencial externo arbitrário não possui solução analítica na mecânica quântica, o que torna o problema de $\mathrm{N}$ partículas ainda mais desafiador. Portanto, é necessário adotar um conjunto de aproximações que simplifiquem a equação 2.2 de forma a torná-la resolúvel, ao menos numericamente.

A equação 2.1 pode ser simplificada adotando-se a aproximação de Born-Oppenheimer, que leva em conta a grande diferença de massa entre os núcleos e os elétrons para desacoplar as equações de movimento e tratar o problema eletrônico separadamente. Assim podemos considerar uma dada configuração dos núcleos, e passar diretamente à equação eletrônica, 


$$
\begin{aligned}
& \mathcal{H}_{\text {elet }} \Phi_{\text {elet }}=\epsilon_{\text {elet }} \Phi_{\text {elet }} \\
& \mathcal{H}_{\text {elet }}=-\sum_{i=1}^{N} \frac{1}{2} \nabla_{i}^{2}-\sum_{i=1}^{N} \sum_{A=1}^{M} \frac{Z_{A}}{\left|\mathbf{r}_{i}-\mathbf{R}_{A}\right|}+\sum_{i=1}^{N} \sum_{j>i}^{N} \frac{1}{\left|\mathbf{r}_{i}-\mathbf{r}_{j}\right|}
\end{aligned}
$$

Resolvendo a equação 2.3 obtem-se as funções de onda eletrônicas que descrevem o movimento dos elétrons, e contém toda a informação relevante sobre as propriedades moleculares tais como momento de dipolo, energias de ionização, polarizabilidades etc. Porém, o termo de repulsão elétron-elétron é ainda muito difícil de determinar, pois envolve o movimento acoplado de todos os elétrons.

Na aproximação de campo médio, para um sistema de N-elétrons, a interação coulombiana entre o $i$-ésimo elétron e a densidade eletrônica produzida pelos outros elétrons N-1 elétrons é descrita pelo potencial de Hartree $V_{H}^{(i)}$ :

$$
V_{H}^{(i)}=\int \frac{n(\mathbf{r})}{\left|\mathbf{r}_{i}-\mathbf{r}\right|} d^{3} \mathbf{r}
$$

onde $n\left(\mathbf{r}_{j}\right)=\sum_{j}\left|\phi_{j}\left(\mathbf{r}_{j}\right)\right|^{2}$ é a densidade eletrônica do sistema.

No método de Hartree-Fock (HF) consideramos

$$
\Phi_{0}^{H F}\left(\mathbf{r}_{1}, \mathbf{r}_{2}, \ldots, \mathbf{r}_{N}\right)=\frac{1}{\sqrt{N !}}\left|\begin{array}{cccc}
\phi_{1}\left(\mathbf{r}_{1}\right) & \phi_{2}\left(\mathbf{r}_{1}\right) & \cdots & \phi_{N}\left(\mathbf{r}_{1}\right) \\
\phi_{1}\left(\mathbf{r}_{2}\right) & \phi_{2}\left(\mathbf{r}_{2}\right) & \cdots & \phi_{N}\left(\mathbf{r}_{2}\right) \\
\vdots & \vdots & \ddots & \vdots \\
\phi_{1}\left(\mathbf{r}_{N}\right) & \phi_{2}\left(\mathbf{r}_{N}\right) & \cdots & \phi_{N}\left(\mathbf{r}_{N}\right)
\end{array}\right|
$$

como a função de onda tentativa na forma de um determinante de Slater [14] que incorpora a propriedade anti-simétrica dos elétrons. Na eq. 2.5. as funções de um elétron $\phi_{i}\left(\mathbf{r}_{j}\right)$ devem ser linearmente independentes e ortogonais entre si, tal que a energia total para o estado fundamental do sistema pode ser escrita como

$$
\begin{aligned}
E_{\text {elet }}^{H F} & =\left\langle\Phi_{0}\left|\mathcal{H}_{\text {elet }}\right| \Phi_{0}\right\rangle=\sum_{i=1}^{N} \int \phi_{a}^{*}\left(\mathbf{r}_{i}\right)\left(-\frac{1}{2} \nabla_{i}^{2}+V_{\text {ext }}\right) \phi_{a}^{*}\left(\mathbf{r}_{i}\right) d^{3} \mathbf{r}_{i}+ \\
& +\frac{1}{2} \sum_{i, j \neq i} \iint \frac{\left|\phi_{a}\left(\mathbf{r}_{i}\right)\right|^{2}\left|\phi_{b}\left(\mathbf{r}_{j}\right)\right|^{2}}{\left|\mathbf{r}_{i}-\mathbf{r}_{j}\right|} d^{3} \mathbf{r}_{i} d^{3} \mathbf{r}_{j}- \\
& -\frac{1}{2} \sum_{i, j \neq i} \iint \phi_{a}^{*}\left(\mathbf{r}_{j}\right) \phi_{b}^{*}\left(\mathbf{r}_{i}\right) \frac{1}{\left|\mathbf{r}_{i}-\mathbf{r}_{j}\right|} \phi_{a}\left(\mathbf{r}_{i}\right) \phi_{b}\left(\mathbf{r}_{j}\right) d^{3} \mathbf{r}_{i} d^{3} \mathbf{r}_{j}
\end{aligned}
$$


onde podemos distinguir o termo puramente coulombiano ou de Hartree, e um termo nãolocal, $V_{x}$, proveniente da antissimetria da função de onda, chamado de interação de troca que não possui análogo clássico. Para $i=j$ o termo de troca cancela exatamente com o termo de Hartree tal que a interação espúria de um elétron com ele mesmo (auto-interação) é naturalmente removida.

Na equação 2.6 vemos que a energia total do sistema de $\mathrm{N}$ partículas depende explicitamente das funções $\phi_{i}$ que, em princípio, são desconhecidas. Este problema é resolvido adotando-se um procedimento iterativo variando sistematicamente o conjunto de orbitais $\phi_{i}$ com o único vínculo de que eles sejam sempre ortonormais. Em particular, o valor esperado de $\mathcal{H}_{\text {elet }}$ satisfaz o princípio variacional tal que a energia do estado fundamental é obtida minimizando a equação 2.6 com respeito às funções de um elétron $\phi_{i}$. O método Hartree-Fock (HF) consiste então em determinar o conjunto de orbitais de uma partícula, $\phi_{i}$, que minimiza a energia total variacionalmente resultando na melhor aproximação possível, de único determinante, para a função de onda do estado fundamental do sistema de $\mathrm{N}$ partículas. Aplicando-se este procedimento chegamos à equação íntegro-diferencial de Hartree-Fock 15, 16,

$$
h\left(\mathbf{r}_{i}\right) \phi_{a}\left(\mathbf{r}_{i}\right)+\left[\sum_{b \neq a} \int \frac{\left|\phi_{b}\left(\mathbf{r}_{j}\right)\right|^{2}}{\left|\mathbf{r}_{i}-\mathbf{r}_{j}\right|} d^{3} \mathbf{r}_{j}\right] \phi_{a}\left(\mathbf{r}_{i}\right)-\left[\sum_{b \neq a} \int \frac{\phi_{b}^{*}\left(\mathbf{r}_{j}\right) \phi_{a}\left(\mathbf{r}_{j}\right)}{\left|\mathbf{r}_{j}-\mathbf{r}_{i}\right|}\right] \phi_{b}\left(\mathbf{r}_{i}\right)=\epsilon_{a} \phi_{a}\left(\mathbf{r}_{i}\right)
$$

onde $h$ é o operador de uma partícula que contém os termos de energia cinética e de energia potencial de atração elétron-núcleo. O primeiro termo entre chaves na eq. 2.7 define também o operador de Coulomb $\hat{J}$ que possui a mesma forma do potencial de Hartree $V_{H}$, e que representa o potencial eletrostático médio sentido por um elétron no orbital $\phi_{i}$ devido à presença dos outros N-1 elétrons que ocupam os outros orbitais. O operador de troca é definido tal que

$$
\hat{K}\left(\mathbf{r}_{i}\right) \phi_{a}\left(\mathbf{r}_{i}\right) \equiv\left[\int \phi_{b}^{*}\left(\mathbf{r}_{j}\right) \frac{1}{\mathbf{r}_{i j}} \phi_{a}\left(\mathbf{r}_{j}\right)\right] \phi_{b}\left(\mathbf{r}_{i}\right) .
$$

Aqui os índices $a$ e $b$ dos spin-orbitais para o elétron $i$ são permutados. $\hat{\mathrm{K}}$ é portanto um operador não-local no sentido de que não existe uma função potencial simples que resulte na ação do operador em um ponto específico $\mathbf{r}_{i}$. O resultado de atuar com $\hat{K}_{b}\left(\mathbf{r}_{i}\right)$ sobre $\phi_{a}\left(\mathbf{r}_{i}\right)$ depende do valor dessa função em todo o espaço, não apenas em $\mathbf{r}_{i}$. Finalmente, a equação 
de Hartree-Fock pode ser escrita na forma de uma equação de autovalores dada em termos dos orbitais de uma partícula $\phi_{i}$,

$$
\hat{F} \phi_{i}=\epsilon_{i} \phi_{i}
$$

onde os autovalores $\epsilon_{i}$ ganham significado físico de acordo com o Teorema de Koopmans, conforme será discutido em detalhes a seguir.

Os cálculos Hartree-Fock para propriedades do estado fundamental de moléculas pequenas normalmente levam a resultados razoavelmente precisos, embora os efeitos de correlação eletrônica sejam desprezados.

\subsubsection{Energia de Ionização: $\triangle \mathrm{SCF}$ e Teorema de Koopmans}

Entre as propriedades de interesse comum no estudo de interfaces orgânico/orgânico e orgânico/metal está a energia de ionização de sistemas moleculares. Esta propriedade está diretamente relacionada às energias de excitação de uma partícula que podem ser medidas através de técnicas de espectroscopia de fotoemissão. Entretanto, a interpretação dos espectros experimentais é muitas vezes difícil de ser realizada sem o auxílio de modelos teóricos precisos.

Formalmente, em um sistema de N-elétrons, a energia necessária para remover um elétron do $s$-ésimo estado do sistema, ou seja, a $s$-ésima energia de ionização catiônica $I$ da molécula é definido como:

$$
I_{s}=E_{s}(N-1)-E(N)
$$

onde $E(N)$ é a energia total do estado fundamental e $E_{s}(N-1)$ é a energia total de um sistema de (N-1)-partículas sendo 1 elétron removido do s-ésimo estado. Tendo em conta (exceto para os gases nobres) a normal "afinidade" atômica para receber um elétron em excesso, define-se a energia para passagem a um estado aniônico como sendo:

$$
A_{s}=E(N)-E_{s}(N+1)
$$

Outra propriedade de interesse é o gap fundamental eletrônico, dado pela diferença

$$
E_{g}=I P-E A
$$


em que se define o potencial de ionização IP como a menor energia de ionização catiônica, e a afinidade eletrônica EA como a menor energia de ionização aniônica; decorre então das equações 2.10 e 2.11 que

$$
E_{g}=E_{s}(N+1)+E_{s}(N-1)-2 E(N)
$$

Portanto, faz-se necessário determinar de forma precisa as energias totais dos sistemas neutro e ionizados tal que a diferença entre esses valores corresponda a energia de excitação eletrônica, o que envolve no mínimo três cálculos completos. Uma alternativa mais eficiente seria estabelecer uma relação entre as energias de ionização e o espectro de autovalores do sistema neutro e isolado.

Ao partirmos formalmente das Eqs. 2.10 2.13 para calcular excitações de um elétron, isto é, energias de ionização e afinidades eletrônicas de sistemas finitos e isolados, temos o método conhecido como $\triangle \mathrm{SCF}[17]$. Neste método, a energia para remover (ou adicionar) um elétron é determinada a partir da diferença na energia do estado fundamental auto-consistente (SelfConsistent Field, SCF) calculada para o sistema neutro e para o íon. Assim, os potenciais de ionização e afinidades eletrônicas obtidas com o método $\triangle \mathrm{SCF}$ incluem efeitos de relaxação de todos os orbitais, levando a resultados em geral precisos para os IPs e EAs.

No contexto da DFT, estudos recentes [18, 19] mostraram que modelos de funcionais padrão podem alcançar uma precisão de 0,1 - 0,2 eV no cálculos dos IPs e EAs utilizando o método $\triangle \mathrm{SCF}$. Entretanto, esta técnica é dificilmente aplicável a sistemas estendidos tais como bulk ou superfícies.

Um procedimento alternativo, específico para IPs e muito utilizado é descrito a seguir, conhecido como Teorema de Koopmans [20].

Na aproximação Hartree-Fock, a energia total do sistema de N-elétrons pode ser escrita, na forma

$$
E(N)=\left\langle\Phi_{N}\left|\mathcal{H}_{\text {elet }}\right| \Phi_{N}\right\rangle=\sum_{a}^{N}\left\langle\phi_{a}\left|h_{1}\right| \phi_{a}\right\rangle+\frac{1}{2} \sum_{a, b}^{N}\left[\left\langle\phi_{a} \phi_{b}\left|h_{2}\right| \phi_{a} \phi_{b}\right\rangle-\left\langle\phi_{b} \phi_{a}\left|h_{2}\right| \phi_{a} \phi_{b}\right\rangle\right]
$$


Seguindo o mesmo raciocínio para o sistema com (N-1)-elétrons, e assumindo que as funções de onda $\Phi_{N}$ e $\Phi_{N-1}$ são compostas pelos mesmos orbitais, a energia necessária para remover um elétron do $s$-ésimo orbital pode ser calculada como segue:

$$
\begin{aligned}
E(N-1)-E(N) & =\sum_{a \neq s}^{N}\left\langle\phi_{a}\left|h_{1}\right| \phi_{a}\right\rangle+\frac{1}{2} \sum_{a, b \neq s}^{N}\left[\left\langle\phi_{a} \phi_{b}\left|h_{2}\right| \phi_{a} \phi_{b}\right\rangle-\left\langle\phi_{b} \phi_{a}\left|h_{2}\right| \phi_{a} \phi_{b}\right\rangle\right] \\
& -\sum_{a}^{N}\left\langle\phi_{a}\left|h_{1}\right| \phi_{a}\right\rangle-\frac{1}{2} \sum_{a, b}^{N}\left[\left\langle\phi_{a} \phi_{b}\left|h_{2}\right| \phi_{a} \phi_{b}\right\rangle-\left\langle\phi_{b} \phi_{a}\left|h_{2}\right| \phi_{a} \phi_{b}\right\rangle\right] \\
E(N-1)-E(N) & =-\left\langle\phi_{s}\left|h_{1}\right| \phi_{s}\right\rangle-\sum_{a}^{N}\left[\left\langle\phi_{a} \phi_{s}\left|h_{2}\right| \phi_{a} \phi_{s}\right\rangle-\left\langle\phi_{s} \phi_{a}\left|h_{2}\right| \phi_{a} \phi_{s}\right\rangle\right]
\end{aligned}
$$

onde utilizamos o fato de que para $a=s$ temos $\left[\left\langle\phi_{s} \phi_{s}\left|h_{2}\right| \phi_{s} \phi_{s}\right\rangle-\left\langle\phi_{s} \phi_{s}\left|h_{2}\right| \phi_{s} \phi_{s}\right\rangle\right]=0$.

$$
I_{s}=E(N-1)-E(N)=-\varepsilon_{s}
$$

Assim, chegamos ao Teorema de Koopmans que afirma que cada autovalor do orbital de Hartree-Fock ocupado dá a energia necessária para remover um elétron do estado de uma partícula correspondente. Uma relação similar é válida também para as afinidades eletrônicas. No contexto da teoria Hartree-Fock, a equação 2.17 é exata apenas na aproximação de orbitais congelados, isto é, sob a suposição de que, após a remoção do s-ésimo elétron de um sistema de $\mathrm{N}$ elétrons, os N-1 orbitais restantes de uma partícula permanecem inalterados (não relaxam) . Em geral, para sistemas finitos e localizados, o acordo entre as energias de ionização de Koopmans no método HF e os valores experimentais é apenas qualitativo. Para átomos e moléculas, a primeira energia de ionização pode apresentar erros de até 2 eV 21,22. Esse não é o caso dos sistemas aqui estudados, como veremos.

As principais fontes de erro relacionadas ao Teorema de Koopmans são a relaxação orbital mencionada acima, que se refere a mudanças no operador de Fock e nos orbitais HF quando o número de elétrons do sistema varia, não tratados pelo Teorema de Koopmans; e a não inclusão de efeitos de correlação eletrônica, que se refere a validade de representar a função de onda completa de muitos corpos como um único determinante de Slater constituído de orbitais de uma partícula. 


\subsection{Teoria do Funcional da Densidade}

A Teoria do Funcional da Densidade (Density Functional Theory - DFT) difere dos métodos baseados na função de onda pelo uso da densidade eletrônica $n(\mathbf{r})$ como quantidade central. Uma importante vantagem em utilizar a densidade eletrônica ao invés da função de onda é a redução na dimensionalidade do problema. Enquanto com funções de onda necessitamos de $3 \mathrm{~N}$ coordenadas para descrever um sistema de N-elétrons, a densidade eletrônica é sempre uma grandeza a três coordenadas independentemente do número de elétrons do sistema. Isto permite que a DFT seja aplicável ao tratamento de sistemas com um grande número de partículas, sendo então possível atualmente estudar sistemas com várias centenas de átomos. Esta característica básica é, em parte, a razão pela qual a DFT se tornou a abordagem mais amplamente utilizada atualmente para cálculos de estrutura eletrônica, particularmente na comunidade de física da matéria condensada, mas também na de química molecular, o que é evidenciado pela concessão do Prêmio Nobel de Química ao físico W. Kohn 23.

Na DFT, o funcional energia de um sistema, dado um potencial externo particular $v$, é definido como segue:

$$
\begin{aligned}
E_{v}[n] & =T[n]+V_{n e}[n]+V_{e e}[n] \\
& =\int n(\mathbf{r}) v(\mathbf{r}) d \mathbf{r}+F_{H K}[n],
\end{aligned}
$$

onde

$$
F_{H K}[n]=T[n]+V_{e e}[n]
$$

Nota-se que $F_{H K}[n]$ depende apenas de $n(\mathbf{r})$ e é independente de qualquer potencial externo $v(\mathbf{r})$. Portanto, $F_{H K}[n]$ é um funcional universal de $n(\mathbf{r})$, que inclui explicitamente a interação elétron-elétron. Assim, o conhecimento da densidade eletrônica $n(\mathbf{r})$ implicaria no conhecimento completo do potencial, e de todas as outras observáveis do sistema. Embora esta sequência descreva a estrutura conceitual da DFT, ela não representa o esquema prático necessário para obter a densidade eletrônica do estado fundamental ou a energia dentro de uma boa aproximação porque o funcional $F_{H} K[n(\mathbf{r})]$ não é especificado. O esquema prático atualmente utilizado para a realização de cálculos baseados na DFT foi desenvolvido posteriormente por Kohn e Sham [24], utilizando um sistema fictício de elétrons não-interagentes 
que se movem na presença de um potencial efetivo, tal que a densidade correspondente seja a mesma de um sistema de elétrons interagentes.

\section{Equações de Kohn-Sham}

O sucesso da Teoria do Funcional da Densidade no tratamento de sistemas atômicos e moleculares se deve, em grande parte, à formulação de Kohn-Sham (KS), que introduz aproximações fundamentais para a densidade eletrônica, e consequentemente para o funcional $F_{H K}[n(\mathbf{r})]$. Kohn e Sham consideraram um sistema de elétrons independentes, ou seja, o conjunto de N elétrons é descrito por uma função produto (o determinante de Slater) de $\mathrm{N}$ "orbitais" sob a ação de um potencial externo efetivo $V^{e f}(\mathbf{r})$. Assim, a idéia central de KS é utilizar a aproximação de "campo médio" no qual o problema original de um sistema de $\mathrm{N}$ elétrons interagentes é reduzido ao problema de uma partícula, sujeito ao vínculo de que a densidade resultante seja igual àquela do sistema interagente. A energia total do sistema interagente é agora escrita como

$$
\begin{aligned}
E[n(\mathbf{r})] & =\int v(\mathbf{r}) n(\mathbf{r}) d \mathbf{r}+F_{H K}[n(\mathbf{r})] \\
& =T_{s}[n(\mathbf{r})]+\int v(\mathbf{r}) n(\mathbf{r}) d \mathbf{r}+\frac{1}{2} \iint n(\mathbf{r}) \frac{1}{\left|\mathbf{r}-\mathbf{r}^{\prime}\right|} d \mathbf{r} d \mathbf{r}^{\prime}+E_{x c}[n(\mathbf{r})],
\end{aligned}
$$

onde $T_{s}[n(\mathbf{r})]$ é a energia cinética do sistema de elétrons não-interagentes com densidade $n(\mathbf{r})$, o termo de Hartree é aquele obtido a partir da função produto, e $E_{x c}[n(\mathbf{r})]$ é a energia de troca e correlação, que contém toda informação sobre os efeitos de muitos corpos, além da correção à energia cinética. Do segundo teorema de Hohenberg-Kohn [25] segue que o funcional energia $E[n(\mathbf{r})]$ não deve variar para pequenos desvios $\delta n(\mathbf{r})$ em torno da densidade exata, e assim a minimização do funcional com respeito a $n(\mathbf{r})$ resulta na seguinte expressão para $V^{K S}(\mathbf{r})$ :

$$
V^{K S}(\mathbf{r})=v(\mathbf{r})+\int \frac{n\left(\mathbf{r}^{\prime}\right)}{\left|\mathbf{r}-\mathbf{r}^{\prime}\right|} d \mathbf{r}^{\prime}+\frac{\delta E_{x c}}{\delta n(\mathbf{r})},
$$

e neste caso a equação de Schroedinger passa a ser escrita como:

$$
\left[-\frac{1}{2} \nabla^{2}+v(\mathbf{r})+\int \frac{n\left(\mathbf{r}^{\prime}\right)}{\left|\mathbf{r}-\mathbf{r}^{\prime}\right|} d \mathbf{r}^{\prime}+v_{x c}(\mathbf{r})\right] \phi_{i}(\mathbf{r})=\varepsilon_{i} \phi_{i}(\mathbf{r})
$$


onde o termo

$$
v_{x c}(\mathbf{r})=\frac{\delta E_{x c}}{\delta n(\mathbf{r})}
$$

é chamado de potencial de troca e correlação e depende de $n(\mathbf{r})$. Esta é uma equação de uma partícula no qual os $\phi_{i}$ são os orbitais de um elétron de Kohn-Sham e os $\epsilon_{i}$ 's são as respectivas energias dos orbitais. Notamos, em comparação com a equação de HartreeFock, que o termo de troca, necessário para o cálculo exato de elétrons independentes, foi absorvido no termo $v_{x c}(\mathbf{r})$. Uma vez que o potencial de Kohn-Sham depende da densidade, as equações 2.22 e 2.21 devem ser resolvidas de forma iterativa e auto-consistente: dada uma função determinantal tentativa inicial, calcula-se o potencial de Kohn-Sham para obter um novo conjunto de orbitais a partir da eq. 2.22, e então determinar uma nova densidade. Este procedimento é repetido até que um certo critério de convergência seja satisfeito. Fazendo um rearranjo para incluir os autovalores na expressão a energia do estado fundamental na aproximação de Kohn-Sham chegamos a:

$$
E=\sum_{i=1}^{N} \varepsilon_{i}-\frac{1}{2} \iint \frac{n(\mathbf{r}) n\left(\mathbf{r}^{\prime}\right)}{\left|\mathbf{r}-\mathbf{r}^{\prime}\right|} d \mathbf{r}^{\prime}+E_{x c}[n]-\int v_{x c}(\mathbf{r}) n(\mathbf{r}) d \mathbf{r}
$$

em que fica clara a importância do funcional de troca e correlação $E_{x c}$. Assim, a precisão dos cálculos DFT está estritamente vinculada à qualidade das aproximações utilizadas para o potencial $v_{x c}$. A seguir, discutiremos duas das principais aproximações mostrando explicitamente a forma do funcional para os dois casos que serão utilizados neste trabalho.

\section{Funcionais de Troca e Correlação}

A primeira forma mais difundida de funcional DFT é a aproximação da densidade local (LDA). Este método baseia-se na suposição de que um sistema real não-homogêneo pode ser dividido em volumes infinitesimais, tal que a densidade eletrônica em cada volume é considerada constante. A energia de troca e correlação do sistema pode ser escrita como:

$$
E_{x c}^{L D A}[n]=\int n(\mathbf{r}) \epsilon_{x c}^{u n i f}(n(\mathbf{r})) d \mathbf{r},
$$

onde $\epsilon_{x c}^{u n i f}$ é a energia exata de troca e correlação por partícula de um gás de elétrons uniforme e interagente com densidade dada por $n(\mathbf{r})$. Na prática, os termos de correlação 
e troca são calculados separadamente. A expressão analítica para a energia de troca é conhecida exatamente:

$$
E_{x}^{L D A}[n]=-\frac{3}{4}\left(\frac{3}{\pi}\right)^{1 / 3} \int n^{4 / 3}(\mathbf{r}) d \mathbf{r}
$$

Quanto ao termo de energia de correlação, nenhuma derivação analítica para o funcional tem se provado possivel, mesmo na aproximação mais simples de um gás de elétrons uniforme. Ceperley e Alder [26] calcularam, utilizando técnicas de Monte Carlo quântico, a energia total de um gás de elétrons uniforme para diferentes densidades em um alto nível de precisão numérica. Então, subtraindo a energia de troca determinada analiticamente para cada caso, pode-se determinar a energia de correlação nestes sistemas. Os funcionais LDA mais modernos tendem a ser bastante semelhantes, diferindo apenas na forma como as contribuições de correlação são ajustadas aos dados obtidos para o gás de elétrons uniforme via métodos de muitos-corpos. Entre os funcionais LDA mais utilizados temos Perdew-Zunger (PZ) 27] e Perdew-Wang (PW) [28] atualmente disponíveis em diversos pacotes computacionais de estrutura eletrônica. Neste trabalho, utilizaremos o funcional LDA-PW.

A LDA é uma aproximação bem sucedida para muitos sistemas de interesse, incluindo metais, moléculas, semicondutores e cristais iônicos. Entretanto, é amplamente reconhecido que a LDA tende a superestimar energias coesivas por $\sim 15-20 \%$ e assim normalmente subestima as constantes de rede em $\sim 2-3 \%$ para seja metais ou isolantes [29, 30].

Em muitos dos materiais semicondutores e isolantes a densidade eletrônica varia rapidamente no espaço, tal que tratar a densidade como localmente uniforme em cada ponto do espaço não é uma boa aproximação para estes casos. Esta aproximação pode, em princípio, ser melhorada adicionando a informação sobre o gradiente da densidade, $\nabla n(\mathbf{r})$. Correções desse tipo são introduzidas na aproximação do gradiente generalizado (GGA) [31, 32], que vai na direção do gás de elétrons não-homogêneo. Os funcionais baseados na aproximação GGA seguem a forma geral:

$$
E_{x c}^{G G A}[n]=\int f^{G G A}(n(\mathbf{r}), \nabla n(\mathbf{r})) d \mathbf{r}
$$

e são frequentemente denominados de "semi-locais" devido à dependência com $\nabla n(\mathbf{r})$. Para muitas propriedades, por exemplo geometrias e energias do estado fundamental de moléculas e sólidos, GGAs podem levar a melhores resultados que os funcionais LDA (mas não sempre, como veremos). Devido à flexibilidade na escolha de $f^{G G A}$, uma enorme variedade de 
funcionais GGA têm sido propostos, os quais podem levar a resultados diversos a depender do sistema e propriedades de interesse 32 .

Dentro da aproximação GGA o termo de troca é escrito como:

$$
E_{x}^{G G A}[n]=\int n(\mathbf{r}) \epsilon_{x}^{u n i f}(n(\mathbf{r})) F_{x}^{G G A}(s) d \mathbf{r}
$$

sendo $F_{x}^{G G A}(s)$ um fator de correção sobre a aproximação do gás de elétrons uniforme $\epsilon_{x}^{u n i f}$. As diferenças entre os funcionais GGA está contida principalmente neste termo de correção $F_{x}^{G G A}(s)$, onde $s$ é

$$
s \propto \frac{|\nabla n(\mathbf{r})|}{n(\mathbf{r})^{4 / 3}}
$$

Ilustramos a forma funcional explícita do termo $F_{x}^{G G A}(s)$ para um dos funcionais GGA mais conhecidos, Perdew-Burke-Ernzerhof (PBE) [31], e que será aplicado neste trabalho. As energias de correlação e troca do funcional PBE (sem incluir polarização de spins) são expressas respectivamente como:

$$
\begin{gathered}
E_{c}^{P B E}[n]=\int n(\mathbf{r})\left[\epsilon_{c}^{u n i f}\left(r_{s}\right)+H\left(r_{s}, t\right)\right] d \mathbf{r}, \\
E_{x}^{P B E}[n]=\int n(\mathbf{r}) \epsilon_{c}^{u n i f}\left(r_{s}\right) F_{x}\left(r_{s}, s\right) d \mathbf{r} .
\end{gathered}
$$

Nas equações 2.30 e 2.31 os termos $H\left(r_{s}, t\right)$ e $F_{x}\left(r_{s}, s\right)$ são funções de dois gradientes adimensionais definidos respectivamente por:

$$
\begin{aligned}
& t=\frac{|\nabla n(\mathbf{r})|}{2 k_{s} n(\mathbf{r})}=\left(\frac{\pi}{4}\right)^{1 / 2}\left(\frac{4}{9 \pi}\right)^{1 / 3} \frac{s}{r_{s}^{1 / 2}} \\
& s=\frac{|\nabla n(\mathbf{r})|}{2 k_{F} n(\mathbf{r})}=\frac{3}{2}\left(\frac{4}{9 \pi}\right)^{1 / 3}\left|\nabla r_{s}\right|
\end{aligned}
$$

A primeira expressão é mais conveniente para descrever a dependência da energia de correlação com o gradiente da densidade eletrônica, enquanto a segunda expressão descreve o termo de troca. As constantes $k_{F}$ e $k_{s}$ que aparecem nas equações 2.32 correspondem, respectivamente, ao vetor de onda de Fermi e o inverso do comprimento de blindagem de Thomas-Fermi de um gás de elétrons com densidade $n(\mathbf{r})$. 


\section{Funcionais Híbridos}

Funcionais híbridos são uma classe de aproximações para o funcional energia de troca e correlação da DFT no qual uma fração da troca semi-local é substituída pela troca exata no sentido Hartree-Fock: assim, já o funcional com troca exata é expresso em termos dos orbitais de Kohn-Sham. A idéia principal dos funcionais híbridos é reduzir o erro de auto-interação presente nos funcionais locais ou semi-locais. Entretanto, os funcionais híbridos envolvem um custo adicional no cálculo do operador de troca não-local, que os torna computacionalmente mais caros que os métodos DFT convencionais retornando, em prática, ao custo de um cálculo HF.

Neste trabalho, estudamos o efeito da troca exata para funcionais híbridos derivados do método PBE (PBEh) são escritos como

$$
E_{x c}^{P B E h}=\alpha E_{x}^{H F}+(1-\alpha) E_{x}^{P B E}+E_{c}^{P B E}
$$

sendo $\mathrm{E}_{x}^{H F}$ a energia de troca do Hartree-Fock (isto é, exata), $\alpha$ um fator de mistura $(0 \leqslant$ $\alpha \leqslant 1), \mathrm{E}_{x}^{P B E}$ a energia de troca e $\mathrm{E}_{c}^{P B E}$ a energia de correlação PBE. Em particular, o funcional híbrido conhecido como PBE0 33, 34 é construído a partir da mistura de $25 \%$ de troca exata HF $(\alpha=1 / 4)$ com $75 \%$ da troca semi-local do PBE. Neste caso, a escolha do fator de mistura é baseada em considerações de teoria de perturbação de muitos corpos de quarta ordem 35.

Outro funcional híbrido bastante popular na comunidade de química quântica é denominado B3LYP, proposto por Becke [36 38]. Este funcional contém três parâmetros determinados empiricamente $\left(a_{0}, a_{1}\right.$ e $\left.a_{2}\right)$ através de um ajuste à dados experimentais de energias de atomização, potenciais de ionização e afinidades protônicas. A energia de troca e correlação é dada por:

$$
E_{x c}^{B 3 L Y P}=a_{0} E_{x}^{H F}+\left(1-a_{0}\right) E_{x}^{L D A}+a_{1} \Delta E_{x}^{B 88}+\left(1-a_{2}\right) E_{c}^{V W N}+a_{2} E_{c}^{L Y P}
$$

onde $\Delta E_{x}^{B 88}$ corresponde a correção do gradiente para a energia de troca dada por Becke88 [39], $E_{x}^{L D A}$ é a energia de troca da LDA, $E_{c}^{V D W}$ é o funcional de correlação de Vosko, Wilk e Nussair [40], e $E_{c}^{L Y P}$ é o funcional de correlação GGA de Lee, Yang e Parr [41]. Os parâmetros utilizados na equação 2.34 são $a_{0}=0,20, a_{1}=0,72$ e $a_{2}=0,81$. Portanto, em relação a fração de troca exata o funcional B3LYP é bastante semelhante ao PBE0. 


\subsection{Teoria de Perturbação Muitos-Corpos: o Método GW}

Em geral, os métodos LDA e GGA tem sido aplicados com sucesso na descrição das propriedades estruturais de moléculas e sólidos, tais como comprimentos de ligação e ângulos, constantes elásticas, propriedades vibracionais [42,43]. Entretanto, a aproximação de campo médio envolvida resulta em uma localização energética incorreta mesmo dos estados eletrônicos ocupados. Esta falha da aproximação KS-DFT é usualmente atribuída ao cancelamento incompleto da auto-interação artificial [27,44] existente nos funcionais. Além disso, os autovalores dos orbitais virtuais (desocupados) não são formalmente providos de qualquer significado físico. Dessa forma o valor do potencial de ionização e da afinidade eletrônica, não são quantidades que podem ser diretamente extraídas dos resultados DFT para o sistema neutro.

A determinação precisa dos níveis eletrônicos de fronteira de sistemas orgânicos como quantidades chaves para o estudo do alinhamento de níveis de energia em interfaces híbridas é uma questão central neste trabalho, tornando-se assim imprescindível passar a métodos para tratar o problema de muitos corpos, e com isso obter a descrição física relevante para os sistemas de interesse. A teoria de função de Green de muitos corpos proporciona a base formal para determinar a estrutura de bandas eletrônica associada à medidas experimentais de fotoemissão. Neste formalismo o sistema original de partículas fortemente interagentes é representado por quasi-partículas fracamente interagentes. A quasi-partícula, de acordo com o conceito de Landau 45, 46], pode ser considerada como uma combinação de um elétron (ou buraco) blindado por uma nuvem de polarização ou, em outras palavras, como uma resposta coletiva do sistema de muitos corpos interagente sobre fotoexcitação.

Na teoria de função de Green, as energias de excitação de uma partícula são soluções da equação de quase-partícula (QP)

$$
\left[-\frac{\nabla^{2}}{2}+v_{e x t}(\mathbf{r})+v_{H}(\mathbf{r})\right] \Psi_{n \sigma}(\mathbf{r})+\int d \mathbf{r}^{\prime} \Sigma\left(\mathbf{r}, \mathbf{r}^{\prime} ; \epsilon_{n \sigma}^{q p}\right) \Psi_{n \sigma}\left(\mathbf{r}^{\prime}\right)=\epsilon_{n \sigma}^{q p} \Psi_{n \sigma}(\mathbf{r})
$$

onde $v_{\text {ext }}$ corresponde ao potencial externo criado pelos núcleos e $v_{H}$ é o termo de repulsão clássico de Hartree; o índice $n$ denota o estado eletrônico e $\sigma$ representa o spin associado. O operador de auto-energia não-local $\Sigma$ contém todos os efeitos da interação elétron-elétron 
além da aproximação de campo médio de Hartree, e pode ser escrito como $\Sigma=G W \Gamma$ onde $G$ é a função de Green de uma partícula, $W$ representa o potencial Coulombiano dinâmicamente blindado, e $\Gamma$ é a função vertex. Esta definição resulta em um conjunto de equações integrodiferenciais acopladas, as equações de Hedin [47], envolvendo $G, W, \Sigma, \Gamma$ e a polarizabilidade $P$ que, em princípio, podem ser resolvidas aplicando-se um procedimento iterativo. Uma vez que a aplicação deste esquema é computacionalmente impraticável, outras aproximações são necessárias. Na prática, considera-se $\Gamma=\delta$ tal que o operador de auto-energia pode ser escrito como:

$$
\Sigma_{\mathrm{GW}}\left(\mathbf{x}, \mathbf{x}^{\prime} ; \epsilon\right)=\frac{i}{2 \pi} \int d \epsilon^{\prime} e^{i \epsilon^{\prime} \eta} G_{0}\left(\mathbf{x}, \mathbf{x}^{\prime} ; \epsilon+\epsilon^{\prime}\right) W_{0}\left(\mathbf{x}^{\prime}, \mathbf{x} ; \epsilon^{\prime}\right),
$$

onde $\mathbf{x}=(\mathbf{r}, \sigma)$ representa as coordenadas coletivas de espaço e spin, e $\eta$ é um número positivo infinitesimal. Em virtude da forma de $\Sigma$ na equação 2.36 esta aproximação ficou popularmente conhecida como "one-shot GW" ou explicitamente $G_{0} W_{0}$. A interação Coulombiana blindada, $W_{0}$, é dada por

$$
W_{0}\left(\mathbf{x}, \mathbf{x}^{\prime} ; \epsilon\right)=\int d \mathbf{x}^{\prime \prime} \varepsilon^{-1}\left(\mathbf{x}, \mathbf{x}^{\prime \prime} ; \epsilon\right) v\left(\mathbf{r}^{\prime \prime}-\mathbf{r}^{\prime}\right)
$$

Nesta equação, $v\left(\mathbf{r}-\mathbf{r}^{\prime}\right)=\frac{1}{\left|\mathbf{r}-\mathbf{r}^{\prime}\right|}$ é a interação Coulombiana pura como no método HF, e $\varepsilon^{-1}\left(\mathbf{x}, \mathbf{x}^{\prime \prime} ; \epsilon\right)$ representa a função dielétrica inversa,

$$
\varepsilon\left(\mathbf{x}, \mathbf{x}^{\prime}, \epsilon\right)=\delta\left(\mathbf{x}-\mathbf{x}^{\prime}\right)-\int d \mathbf{x}^{\prime \prime} v\left(\mathbf{r}-\mathbf{r}^{\prime \prime}\right) P_{0}\left(\mathbf{x}^{\prime \prime}, \mathbf{x}^{\prime} ; \epsilon\right)
$$

que é obtida da polarizabilidade

$$
P_{0}\left(\mathbf{x}, \mathbf{x}^{\prime} ; \epsilon\right)=-\frac{i}{2 \pi} \int d \epsilon^{\prime} e^{\epsilon^{\prime} \eta} G_{0}\left(\mathbf{x}, \mathbf{x}^{\prime} ; \epsilon+\epsilon^{\prime}\right) G_{0}\left(\mathbf{x}^{\prime}, \mathbf{x} ; \epsilon^{\prime}\right)
$$

Finalmente, a função de Green não-interagente $G_{0}$ é contruída a partir das auto-energias e auto-funções de um cálculo HF ou DFT anterior

$$
G_{0}\left(\mathbf{x}, \mathbf{x}^{\prime} ; \epsilon\right)=G_{0}^{\sigma}\left(\mathbf{r}, \mathbf{r}^{\prime} ; \epsilon\right)=\sum_{n} \frac{\Psi_{n \sigma}(\mathbf{r}) \Psi_{n \sigma}^{*}\left(\mathbf{r}^{\prime}\right)}{\epsilon-\left(\epsilon_{n \sigma}+i \eta \operatorname{sgn}\left(\epsilon_{F}-\epsilon_{n \sigma}\right)\right)} .
$$

Com isto, a equação da quase-partícula (Eq. 2.35) torna-se

$$
\epsilon_{n \sigma}^{q p}=\epsilon_{n \sigma}^{\mathrm{KS}}+\Re\left\langle\Psi_{n \sigma}\left|\Sigma^{G W}\left(\epsilon_{n \sigma}^{q p}\right)-v_{x c}\right| \Psi_{n \sigma}\right\rangle=\epsilon_{n \sigma}^{\mathrm{KS}}+\Delta_{n \sigma}^{q p}
$$


onde $v_{x c}$ é o potencial de troca e correlação do cálculo DFT (or HF) inicial e $\Delta_{n \sigma}^{q p}=$ $\Re\left\langle\Psi_{n \sigma}\left|\Sigma^{G W}\left(\epsilon_{n \sigma}^{q p}\right)-v_{x c}\right| \Psi_{n \sigma}\right\rangle$ é a correção $G_{0} W_{0}$ ou de quase-partícula. As equações 2.36 2.41 ilustram que $\Delta_{n \sigma}^{q p}$, e portanto as energias de quase-partícula, dependem do funcional DFT utilizado no cálculo precedente. Isto é conhecido na literatura 48 50 como dependência do cálculo $G_{0} W_{0}$ com o ponto de partida ou starting point dependence. Assim, ressaltamos que na aproximação $G_{0} W_{0}$, os orbitais e a densidade eletrônica são os mesmos obtidos no cálculo DFT precedente, apenas as auto-energias são corrigidas pelo tratamento perturbativo dos efeitos de correlação, podendo inclusive modificar o ordenamento dos níveis de energia uma vez que a correção GW depende da simetria particular de cada estado.

Os modelos teóricos e aproximações discutidas neste capítulo estão atualmente implementados em diversos pacotes computacionais utilizados em cálculos de estrutura eletrônica. Neste caso, o uso de algorítmos computacionais eficientes é imprescindível para o estudo quântico de sistemas complexos com centenas ou milhares de átomos, permitindo alcançar um alto nível de precisão numérica para as propriedades de interesse. Aqui, selecionamos como ferramenta computacional o programa "FHI-AIMS" (The Ab initio Molecular Simulaions Package) que possui uma estrutura bastante eficiente e preenche os requisitos básicos para o desenvolvimento deste trabalho. A descrição completa e detalhada do conjunto de algorítmos implementados no "FHI-AIMS" pode ser encontrada na referência [51], e apresentamos no apêndice B uma breve descrição das principais funcionalidades do programa e aproximações (conjuntos de base) utilizadas nesta Tese. 


\section{Capítulo 3}

\section{Estrutura Eletrônica do Sistema Conjugado Modelo: Poliacetileno}

O trans-poliacetileno (TPA) é desde o início considerado como um excelente sistema modelo devido à simplicidade de sua estrutura e também por apresentar algumas propriedades básicas que são comuns aos demais polímeros conjugados conhecidos. Assim sendo, o TPA tem sido estudado através de diferentes formalismos [52 54], e também experimentalmente tanto para oligômeros pequenos quanto para filmes poliméricos condutores [55,56]. Aqui, após obtermos um modelo estrutural para o TPA a partir de cálculos de otimização DFT, construímos uma série de oligômeros-modelo de comprimento crescente e analisamos, ainda na aproximação de campo médio, a evolução das propriedades eletrônicas como função do comprimento molecular. Em seguida, calculamos a estrutura eletrônica dos oligômeros utilizando teoria de perturbação de muitos corpos dentro da aproximação $G_{0} W_{0}$ e a seguir aplicamos um procedimento de consistência interna para ajustar o parâmetro de troca-exata do funcional híbrido PBEh. Todos os cálculos de estrutura eletrônica foram realizados com o pacote computacional "all-electron" FHI-aims [51].

A análise dos valores obtidos para o potencial de ionização vertical levam a um resultado muito interessante, já que apesar de não ter sido imposto nenhum vínculo, os erros relativamente aos valores experimentais são consideravelmente reduzidos. Entretanto, ressaltamos que o objetivo principal deste capítulo não é a reprodução ou previsão de resultados experimentais, mas sim estudar o comportamento de propriedades eletrônicas com o alcance de interações incluídas nos formalismos teóricos: essa é a motivação maior para a escolha dos 
trans-acetilenos como objeto de estudo, seja na forma de oligômeros ou polímero infinito, devido à sua característica quase-unidimensional.

\subsection{Resultados de campo médio padrão: DFT vs. Hartree-Fock}

Iniciamos pela discussão dos resultados obtidos através dos métodos de Hartree-Fock e DFT no formalismo de Kohn-Sham (KS-DFT). Determinamos primeiramente a geometria de equilíbrio de uma cadeia isolada de TPA, sujeita a condições periódicas de contorno, com constante de rede fixa. Os cálculos de relaxação estrutural foram realizados com o funcional PBE [31, incluindo correções de van der Waals no modelo de Tckatchenko-Scheffler [57]. Neste caso o comprimento do monômero de TPA, definido pela constante de rede paralela à direção de crescimento da cadeia, foi fixado no valor experimental determinado por medidas de Raios-X para a fase cristalina [58], $\mathrm{c}=2,457 \AA$. Os demais parâmetros da célula unitária foram fixados em $\mathrm{a}=\mathrm{b}=25 \AA$ para minimizar as interações entre imagens periódicas, simulando uma cadeia unidimensional isolada. Utilizamos uma malha regular de pontos-k de 1x1x10 para representar as funções de onda na rede recíproca.

A estrutura otimizada do TPA apresenta distâncias de ligação carbono-carbono $\mathrm{d}_{(C=C)}$ $=1,362 \AA$ para a ligação dupla e $\mathrm{d}_{(C-C)}=1,423 \AA$ para a ligação simples, o que dá origem à dimerização da cadeia polimérica. O comprimento de ligação C-H obtido é $1.095 \AA$ e o ângulo entre carbonos C-C-C é 123,8. Cálculos anteriores ab initio incluindo correlação eletrônica no nível MP2 $\left[59 \mid\right.$ resultaram em $\mathrm{d}_{(C=C)}=1,378 \AA$ e $\mathrm{d}_{(C-C)}=1,436 \AA$ para a cadeia infinita de poliacetileno, enquanto os valores DFT/PBE0 60 são $\mathrm{d}_{(C=C)}=1,369$ $\AA \mathrm{e}_{(C-C)}=1,425 \AA$. Vemos portanto que a geometria de equilíbrio do TPA obtida por nossos cálculos $\mathrm{PBE}+\mathrm{vdW}$ é bastante similar às estruturas reportadas em trabalhos teóricos anteriores, e também está em bom acordo com outros dados experimentais [61].

Uma vez que o nosso foco é sobre a evolução das propriedades eletrônicas com o comprimento molecular, utilizamos a estrutura otimizada do TPA como base para construir uma série de oligômeros conjugados lineares (OTAs) com o número de ligações duplas variando entre $n=1$ (etileno) e $n=50$, que corresponde a um comprimento máximo de $12 \mathrm{~nm}$. Sendo assim, mantemos fixas as posições atômicas relativas dos átomos "internos" a cada oligômero conforme obtido no cálculo de relaxação do TPA, e otimizamos apenas as distâncias C-H dos 
grupos terminais $\mathrm{CH}_{2}$. Calculamos a estrutura eletrônica de cada oligômero com diferentes funcionais LDA-PZ, PBE e também com o híbrido PBE0. Ainda na aproximação de campo médio, calculamos as propriedades eletrônicas do estado fundamental dos OTAs utilizando o método Hartree-Fock.

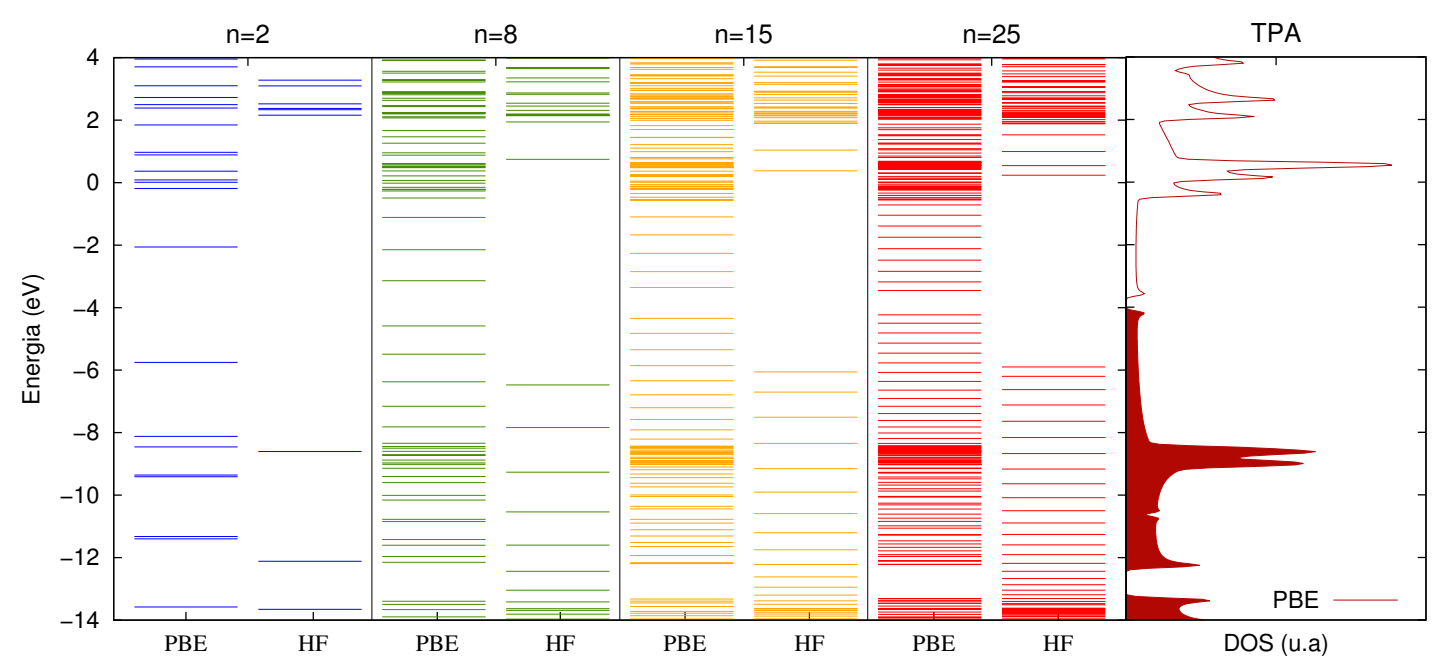

Figura 3.1: Evolução do espectro de níveis de energia DFT/PBE e HF calculados para oligômeros isolados de trans-acetilenos OTAs, e perfil da densidade de estados calculada para cadeia polimérica 1D.

Analisando primeiramente os resultados obtidos com o funcional PBE padrão, representamos na Fig. 3.1 a evolução no espectro de níveis de energia para uma série crescente de oligoacetilenos comparando-os com a distribuição contínua de estados eletrônicos, DOS, calculada para o polímero infinito. Para alinhar os níveis de energia do sistema periódico com os das moléculas isoladas, utilizamos o valor médio da energia dos estados de caroço $\mathrm{C}_{1 s}$, e o igualamos ao valor médio para os estados do oligômero mais longo, $\mathrm{n}=50$.

Qualitativamente, notamos que os níveis eletrônicos dos oligômeros evoluem gradualmente para formar um contínuo de estados ocupados e desocupados correspondentes às bandas de energia da cadeia isolada de TPA ( $\pi$ e $\pi^{*}$ nessa região graficada). Vemos ainda uma nítida diminuição no gap HOMO-LUMO com o aumento da cadeia, convergindo para $E_{\text {gap }} \simeq 0.54 \mathrm{eV}$ no cálculo explícito do polímero isolado. Incluimos também na mesma figura os resultados obtidos através do método HF para esta série de OTAs, e vê-se a grande diferença entre os gaps. Como visto no capítulo anterior, os autovalores de funcionais de campo médio não são qualificados para fornecer energias envolvendo estados virtuais ou de- 
socupados, ou para transições (no caso do gap); entretanto, seria interessante poder obter estimativas razoáveis a partir do resultado HOMO-LUMO, que de fato é muito utilizado para tal. A DFT, como se espera, subestima o gap fundamental do TPA quando comparado a cálculos GW $\left(E_{\text {gap }}=2.1 \mathrm{eV}\right)[12$ ou mesmo se comparado ao gap ótico experimental de 1,4 - 1,8 eV 62,63. devido ao erro de auto-interação. Já o método Hartree-Fock tende a superestimar esse gap, o que é também reconhecido para outros sistemas conjugados com valores tipicamente da ordem de 6,0 a 7,0 eV [52,64]. Funcionais híbridos com uma fração constante $(\alpha)$ de troca-exata melhoram a descrição do gap de energia [65], porém a escolha do parâmetro $\alpha$ não é unívoca e, em geral, depende do sistema [66.

Focamos agora na energia do HOMO de pequenos oligômeros, verificando a adequação do teorema de Koopmans para o potencial de ionização vertical $\left(\operatorname{IP}_{v}=-\epsilon_{H O M O}\right)$. Na tabela 3.1 listamos os resultados para o IP das menores moléculas da série de OTAs, para os quais dados experimentais de fase gasosa são conhecidos. Calculamos os IPs utilizando diferentes funcionais da densidade (LDA-PZ, PBE e o híbrido PBE0), e também com o método HartreeFock (Teorema de Koopmans). Comparando nossos resultados com dados da literatura teórica (B3LYP) e experimental, vemos que os métodos DFT subestimam os IPs de fase gasosa para todos os oligômeros. A adição de uma pequena fração de troca-exata nos funcionais híbridos tende a baixar a energia do HOMO melhorando a descrição dos IPs. Porém, entre os modelos de campo médio considerados, apenas o método HF resulta em um bom acordo com os dados experimentais. Confirmamos assim que a energia do HOMO é bastante sensível aos efeitos não-locais da troca exata, especialmente nas menores moléculas conjugadas onde o IP pode aumentar em até $\sim 3,5 \mathrm{eV}$ quando passamos do PBE ao método HF.

Na tabela 3.2 listamos os valores teóricos do gap HOMO-LUMO para as mesmas moléculas. Para determinar o valor do gap fundamental precisamos além do primeiro potencial de ionização também a primeira afinidade eletrônica $\left(\mathrm{E}_{g a p}=\mathrm{IP}-\mathrm{EA}\right)$. Devido às dificuldades em medir a eletroafinidade, pudemos incluir na tabela 3.2 os valores experimentais de referência apenas para os dois menores oligômeros da família de OTAs. Comparando os resultados obtidos por diferentes modelos teóricos, notamos que as tendências nos valores dos gaps são bastante similares àquelas já esperadas: os métodos DFT convencionais subestimam o gap dos oligômeros conjugados, e novamente, o método HF resulta no melhor acordo com os dados experimentais entre todos os modelos de campo médio avaliados. 


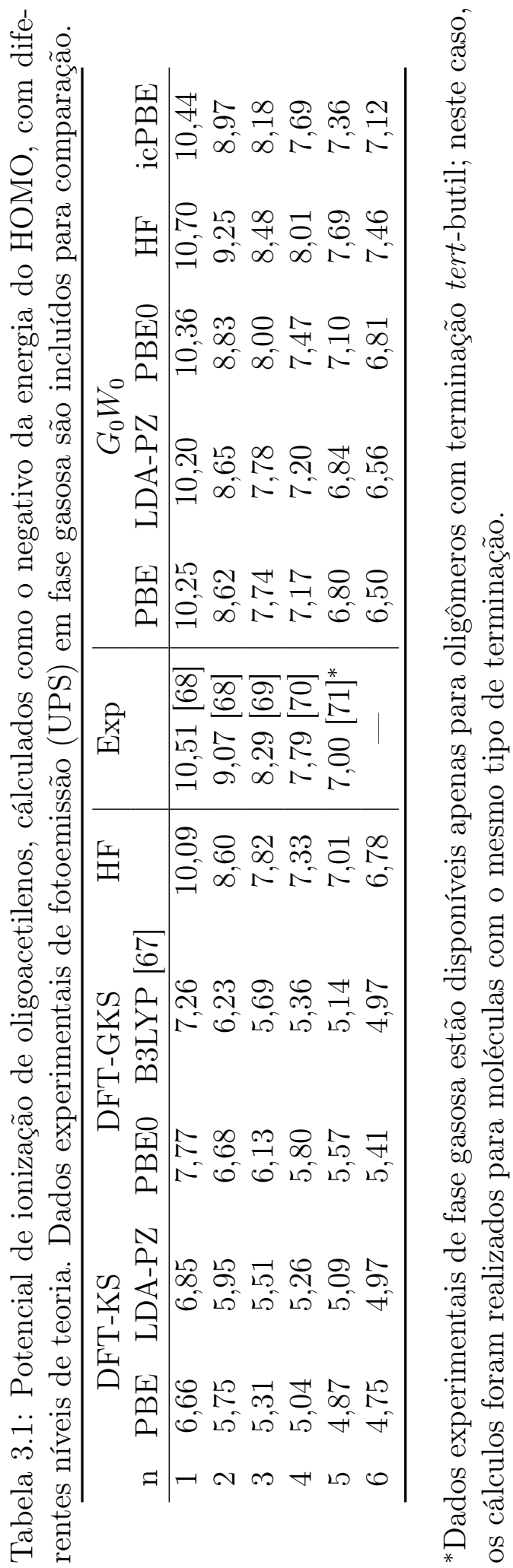




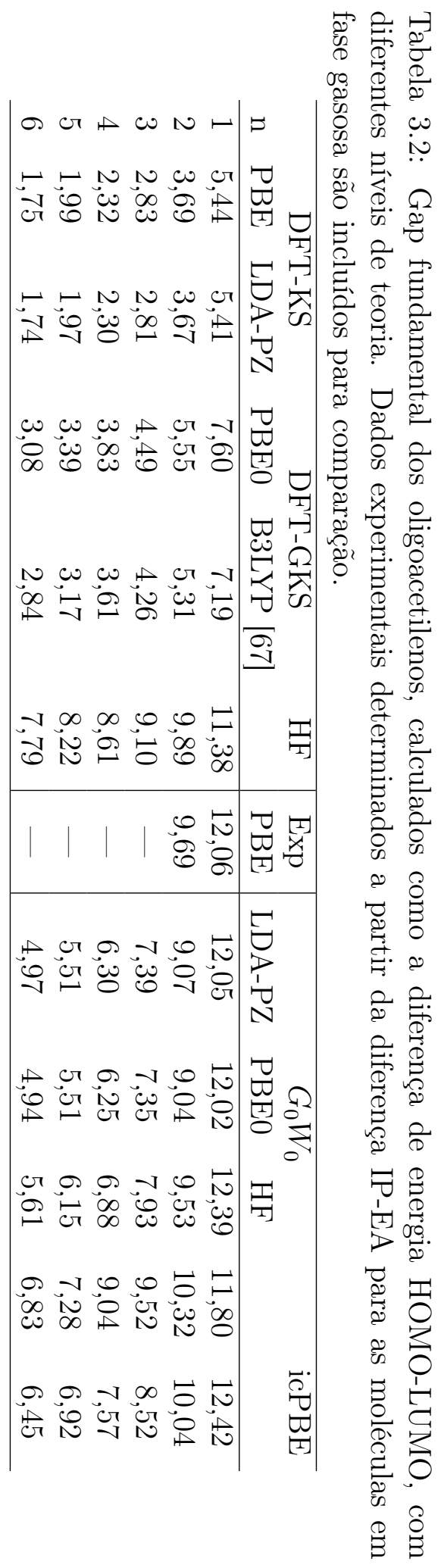


Passamos agora aos resultados de campo médio para os IPs utilizando o cálculo $\triangle \mathrm{SCF}$, que como descrito anteriormente leva em consideração a relaxação orbital, e tem-se provado bastante preciso na determinação dos IPs e gaps fundamentais de sistemas tais como átomos, clusters $|72|$ e pequenas moléculas $\left[73\right.$. Além disso, o $\mathrm{IP}_{\triangle S C F}$ tem sido também utilizado para alinhar o espectro de autovalores DFT (por deslocamento rígido a partir do HOMO) melhorando o acordo com os dados experimentais de fotoemissão [74|. Entretanto, estudos recentes 75,76 mostraram que o uso de funcionais DFT padrão leva a falhas severas no cálculo dos IPs quando aplicado a cadeias conjugadas longas, mesmo quando aplicados na aproximação DFT- $\Delta$ SCF: essa conclusão (inadequação) vem normalmente da comparação com dados experimentais, porém o que destacamos aqui é que a diferença entre o resultado direto $\left(\mathrm{IP}=-\varepsilon_{\text {HOMO }}\right)$ e o resultado $\Delta \mathrm{SCF}$ diminui progressivamente com o comprimento da cadeia. De fato, mostramos na Fig. 3.2 a dependência dos IPs com o comprimento molecular para a série de OTAs, obtidos com o funcional PBE. Vemos que a diferença entre o $\mathrm{IP}_{\triangle S C F} \mathrm{e}$

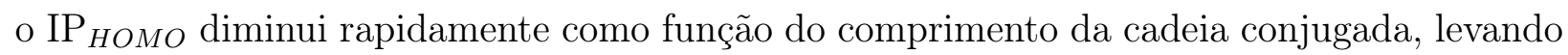
a valores muito próximos para o IP da cadeia $n=50$, que como vimos na Fig. 3.1 converge para o valor do HOMO do sistema infinito. Este comportamento se deve ao fato de que a variação na energia total devido à remoção (ou adição) de um elétron torna-se muito pequena em sistemas com um número grande de elétrons ( $\mathrm{N} \gg 1)$ altamente delocalizados, como se espera em um sistema de conjugação $\pi$ (o que justifica a análise de Janak). Aqui, em prática, essa variação passa de 3,0 eV para comprimentos da ordem de 0,4 nm a 0,6 eV para moléculas com $12 \mathrm{~nm}$.

\subsection{Otimizando funcionais híbridos para sistemas conju- gados}

Discutimos agora os resultados para os mesmos sistemas, obtidos com aplicação da perturbação $\mathrm{G}_{0} \mathrm{~W}_{0}$. Como já mencionado, a correção de auto-energia $\Sigma$ para os estados eletrônicos obtida de forma não-auto-consistente, $\mathrm{G}_{0} \mathrm{~W}_{0}$, pode resultar em energias de quasipartícula, bem como o ordenamento desses níveis eletrônicos, dependentes do modelo teórico subjacente. Assim, realizamos cálculos $\mathrm{G}_{0} \mathrm{~W}_{0}$ sobre a densidade eletrônica do estado fundamental obtida com diferentes métodos (DFT e HF) e listamos também os resultados na tabela 3.1. Notamos que a correção de auto-energia introduzida perturbativamente via cál- 


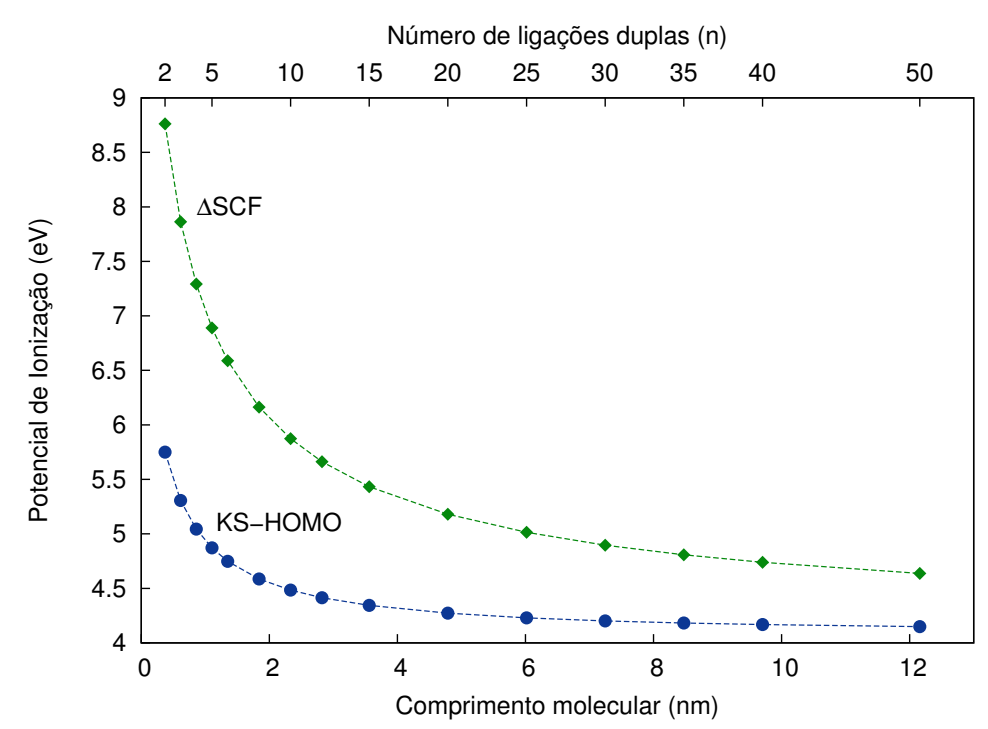

Figura 3.2: Evolução dos IPs da família de oligoacetilenos calculados com os métodos $\Delta \mathrm{SCF}$ e Koopmans $\left(-\epsilon_{\text {HOMO }}\right)$ utilizando o funcional PBE padrão.

culo $\mathrm{G}_{0} \mathrm{~W}_{0}$ promove, em todos os casos, um abaixamento do nível HOMO, e que as diferenças observadas nas energias de quasipartícula devido às diferentes condições iniciais no cálculo GW são significativamente menores do que as diferenças nos valores do HOMO entre os métodos originais DFT ou HF. Entretanto, pode-se notar que estas diferenças aumentam com o tamanho dos oligômeros, ou seja, a tendência se inverte: enquanto no caso de campo médio as diferenças passam de $\sim 3,5 \mathrm{eV}$ para $\mathrm{n}=2$ a $\sim 2,0 \mathrm{eV}$ para $\mathrm{n}=6$, agora passamos de $\sim 0,5 \mathrm{eV}$ para $\mathrm{n}=2$ chegando $\mathrm{a} \sim 1,0 \mathrm{eV}$ para $\mathrm{n}=6$. Isto reforça a necessidade de encontrar um método DFT que melhor represente as funções de onda de quasipartícula em sistemas $\pi$-estendidos, para um posterior cálculo $\mathrm{G}_{0} \mathrm{~W}_{0}$.

A estratégia que adotamos aqui, originalmente proposta na referência [77, baseia-se na escolha do parâmetro de mistura ou fração $\alpha$ de troca exata dos funcionais híbridos que resulte na melhor descrição possível para o potencial de ionização dos sistemas de interesse. O procedimento consiste em variar o parâmetro $\alpha$ tal que a correção de quasipartícula $\Delta_{\text {HOMO }}^{q p}$ para o nível HOMO seja minimizada. Reforçando nosso argumento, ao minimizarmos $\Delta_{\mathrm{HOMO}}^{q p}$ exigimos que a correspondência entre HOMO e IP válida na DFT exata seja satisfeita [78,79. Desta forma, eliminamos a ambiguidade na escolha do $\alpha$, obtendo assim 
um potencial de troca e correlação no formalismo de Kohn-Sham generalizado internamente consistente (ic), sem ajustes derivados de dados experimentais.

Na prática, utilizamos o funcional híbrido de Perdew, Burke and Ernzerhof [35] (PBEh) e realizamos uma série de cálculos para diferentes valores de $\alpha$. Os orbitais e auto-valores de Kohn-Sham resultantes são então adotados como entrada para os cálculos $\mathrm{G}_{0} \mathrm{~W}_{0} @ \mathrm{PBEh}$ subsequentes. A dependência das energias de quase-partícula e, respectivamente, as energias de partida DFT podem assim ser analisadas para determinar o parâmetro $\alpha$ de troca-exata para o qual $\Delta_{\text {HOMO }}^{q p}$ se anula. Verificamos que essa determinação pode ser obtida com apenas alguns poucos cálculos $\mathrm{G}_{0} \mathrm{~W}_{0}$, o que torna o nosso procedimento razoavelmente eficiente.

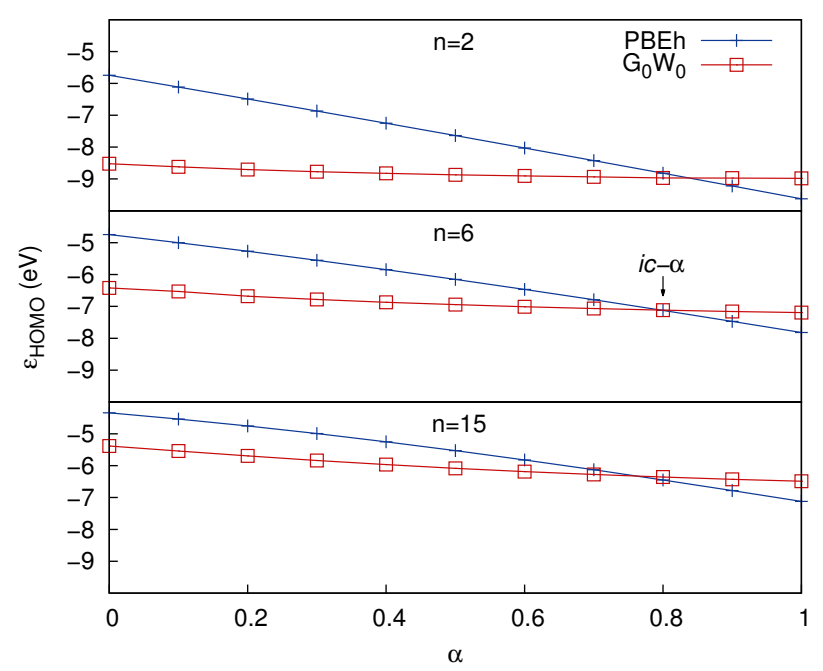

Figura 3.3: Evolução da energia do KS-HOMO e da energia de quasipartícula correspondente como função do percentual de troca exata inserido no funcional híbrido PBEh. O parâmetro $\alpha$ que satisfaz o critério de consistência interna, indicado no painel central, corresponde ao ponto de cruzamento entre as curvas calculadas com PBEh e $\mathrm{G}_{0} \mathrm{~W}_{0} @ \mathrm{PBEh}$.

Mostramos na Fig. 3.3 nossos resultados para a energia do HOMO DFTh e a energia da quasi-partícula para o mesmo estado, realizado dentro dessa estratégia. Devido ao (ainda alto) custo computacional, utilizamos neste caso o conjunto de base tier 2, porém alguns testes realizados com uma base maior não resultaram em maiores comprometimentos na qualidade dos resultados obtidos. Qualitativamente, é importante notar que a energia de quasipartícula do HOMO vinda dos cálculos $\mathrm{G}_{0} \mathrm{~W}_{0}$ é bastante estável com respeito ao parâmetro $\alpha$, ao passo que a energia DFTh-HOMO varia significativamente quando passamos do funcional PBE puro $(\alpha=0)$ para o funcional com $\alpha=1$. Os pontos de interseção entre 
as curvas PBEh e $\mathrm{G}_{0} \mathrm{~W}_{0}$, que indicam o percentual consistente de troca exata - $i c$ - $\alpha$ - para cada oligômero, ocorre em torno de $\alpha \approx 0.80$. Este valor é portanto muito maior do que a fração EX incluída nos funcionais híbridos tradicionais tais como B3LYP, HSE ou PBE0.

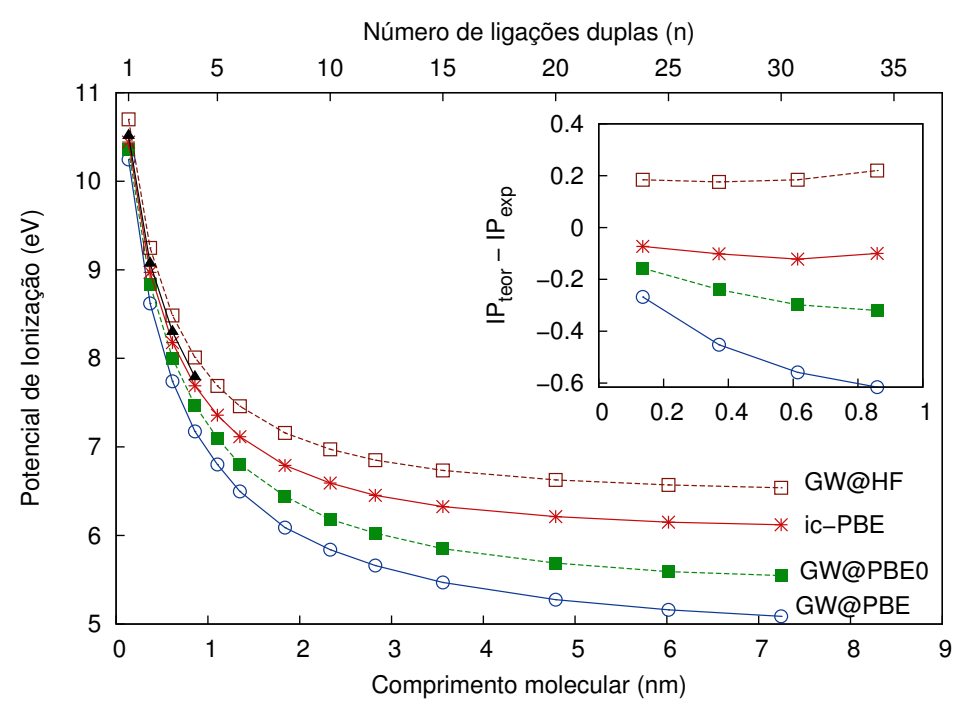

Figura 3.4: Energia de ionização vertical como função do comprimento molecular de oligômeros de trans-acetileno calculados na aproximação $\mathrm{G}_{0} \mathrm{~W}_{0}$ sobre diferentes níveis de teoria: PBE (círculos), PBE0 (quadrados sólidos) and ic-PBE (estrelas); Hartree-Fock (quadrados vazios). Incluímos também os dados experimentais (triângulos sólidos) para pequenos oligômeros. Inset: desvio dos IP's em relação ao valor experimental de fase gasosa.

Ainda na tabela 3.1 listamos os valores dos IPs calculados para oligoacetilenos com $\mathrm{n}=1$ a 6 de acordo com o nosso critério de consistência interna $P B E h \leftrightarrow G_{0} W_{0}$. Vemos então que os resultados obtidos com o funcional ic-PBE apresentam uma boa correlação com os potenciais de ionização medidos em fase gasosa, embora nenhum vínculo adicional tenha sido imposto em nosso modelo para reproduzir o experimento. É interessante notar que, em um estudo teórico recente [80] realizado para um conjunto de oligômeros conjugados foi mostrado que, entre os diferentes funcionais de troca-correlação utilizados, o BHandH com $50 \%$ de troca exata resultou no melhor acordo com os valores de IP experimentais.

Uma vez que estamos interessados principalmente na descrição das propriedades eletrônicas de sistemas poliméricos estendemos a nossa análise dos IPs a cadeias conjugadas mais longas. Mostramos na Fig. 3.4 os IPs versus comprimento molecular calculados com o método $\mathrm{G}_{0} \mathrm{~W}_{0}$ para dois funcionais padrão, PBE e PBE0, e HF, e também com o funcional ic-PBE (que representa o resultado $\mathrm{G}_{0} \mathrm{~W}_{0} @$ @ic-PBE por construção) para oligoacetilenos com 
$\mathrm{n}=1-30$. Novamente, os valores experimentais para moléculas pequenas também são incluídos para comparação. Primeiramente, nossos cálculos reforçam a dependência dos resultados GW com o estado fundamental de referência DFT ou HF, especialmente para cadeias mais longas $(l>5 \mathrm{~nm})$ onde os valores dos IPs podem diferir em até $1,5 \mathrm{eV}$ a depender do funcional adotado. Em particular, notamos que a saturação no valor dos IPs ocorre para cadeias mais curtas quando métodos de campo médio com maior contribuição de troca-exata são utilizados para iniciar o cálculo $\mathrm{G}_{0} \mathrm{~W}_{0}$. Enquanto os desvios em relação aos valores experimentais nos IPs obtidos a partir de cálculos GW aumentam como função do comprimento molecular, estes erros se mantém aproximadamente constantes e próximos de zero quando utilizamos o funcional ic-PBE, conforme visto no inset da Fig. 3.4. Isto se deve ao fato de que o grau de blindagem eletrônica ou screening nos sistemas conjugados 1D depende do comprimento molecular, e portanto o parâmetros ic- $\alpha(l)$ obtido por meio do critério de consistência interna também devem variar de acordo com o tamanho de cada oligômero. Assim, analisamos em detalhes na Fig. 3.5 a dependência do parâmetro de troca-exata ic- $\alpha$ com o comprimento dos oligoacetilenos.

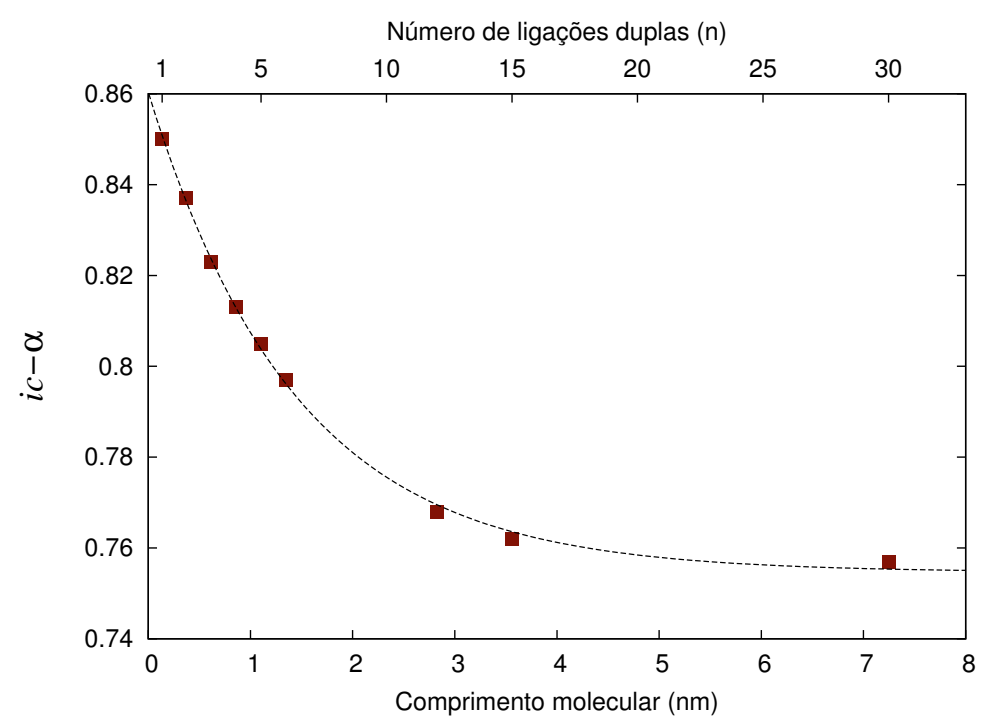

Figura 3.5: Evolução do parâmetro $\alpha$, otimizado de acordo com critério de consistência interna, com o comprimento da cadeia dos oligômeros de trans-acetileno. A curva tracejada de ajuste indica que o $\alpha$ decresce exponencialmente com o comprimento da cadeia: ic$\alpha(l)=0.106 e^{-0.694 l}+0.755$. 
Notamos que o parâmetro de troca-exata consistente varia pouco com o tamanho dos oligômeros, assumindo um valor máximo de $\alpha \approx 0,85$ para a molécula de etileno $(\mathrm{n}=1)$ e alcançando o seu valor mínimo $\alpha \approx 0,76$ para a molécula com $\mathrm{n}=30$ ligações duplas. Em particular, a função ic- $\alpha(l)$ ajustada decresce exponencialmente até um valor de saturação, conforme indicado pela curva de ajuste na Fig. 3.5. Como mencionado acima, este comportamento reflete a variação na blindagem eletrônica ao passarmos de sistemas moleculares confinados (0D) para sistemas $\pi$ estendidos (1D). De fato, sistemas moleculares apresentam grandes valores de gap, e portanto a blindagem dielétrica é muito fraca, tal que para esses sistemas a fração de troca-exata correta deve se aproximar do limite HF. Já em sistemas $\pi$ estendidos os estados de fronteira são delocalizados e facilmente polarizáveis, resultando em uma blindagem mais intensa e, portanto um menor parâmetro de troca-exata. Extrapolando a curva de ajuste exponencial obtida para ic- $\alpha(l)$ estimamos a fração ideal de troca-exata no funcional PBEh como sendo $75 \%$ para uma cadeia isolada de TPA.

Em uma publicação recente [81, Refaely-Abramson et al também propuseram um esquema para aproximar os resultados DFT da versão apropriada que obedece à postulação Janak-Koopmans, naquele caso ajustando o parâmetro que controla o alcance da interação coulombiana no funcional híbrido. Aplicando este esquema a uma série de semicondutores orgânicos de tamanhos distintos, os autores verificaram que o valor do parâmetro ótimo $\gamma$ também diminui com o aumento do tamanho molecular, uma tendência previamente observada também para nanocristais de silício e oligoacenos 82 .

A análise qualitativa dos orbitais moleculares de fronteira pode fornecer uma compreensão mais clara da influência da troca-exata sobre a estrutura eletrônica dos oligômeros conjugados. Assim, mostramos na tabela gráfica 3.3 a densidade eletrônica do HOMO para a molécula OTA(30) obtida com o funcional PBEh utilizando diferentes percentuais de troca-exata. Vemos que o aumento do parâmetro $\alpha$ resulta em um progressivo aumento na localização espacial do HOMO. De fato, é bem conhecido que, na aproximação semi-local a interação coulombiana de um elétron com ele mesmo favorece a delocalização do orbital de Kohn-Sham correspondente 83], um efeito que tende a ser exacerbado para sistemas estendidos 84. Isto está também em acordo com o comportamento decrescente do parâmetro ic- $\alpha$ vs $l$, visto que a quantidade de troca-exata necessária para cancelar os erros espúrios de auto-interação (self-interaction error SIE) deve diminuir para sistemas $\pi$-estendidos.

Uma importante propriedade da KS-DFT exata demonstrada por Perdew et al. 85, 86 é que a energia total de um dado sistema deve ser linear com relação a um número fracionário 
Tabela 3.3: Densidade eletrônica calculada para os orbitais moleculares de fronteira (KSLUMO e KS-HOMO) de um oligacetileno com $n=30$ ligações duplas. As isosuperfícies foram obtidas com o funcional PBEh utilizando diferentes valores para a fração de troca-exata, variando entre $\alpha=0$ até $1 ; \alpha=0.757$ corresponde ao parâmetro ótimo obtido com o critério de consistência interna.

\begin{tabular}{|c|c|c|}
\hline$\alpha$ & & Densidade Eletrônica \\
\hline 0.000 & $\begin{array}{l}\text { LUMO } \\
\text { HOMO }\end{array}$ & 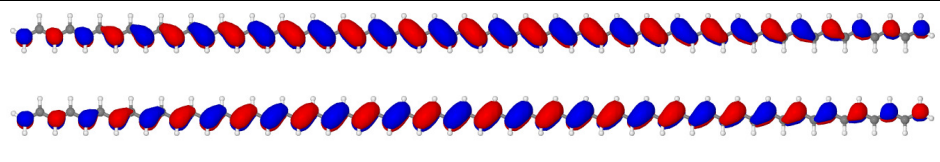 \\
\hline 0.400 & $\begin{array}{l}\mathrm{LUMO} \\
\mathrm{HOMO}\end{array}$ & 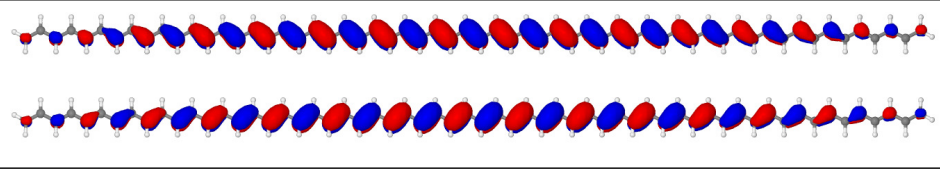 \\
\hline 0.757 & $\begin{array}{l}\text { LUMO } \\
\mathrm{HOMO}\end{array}$ & 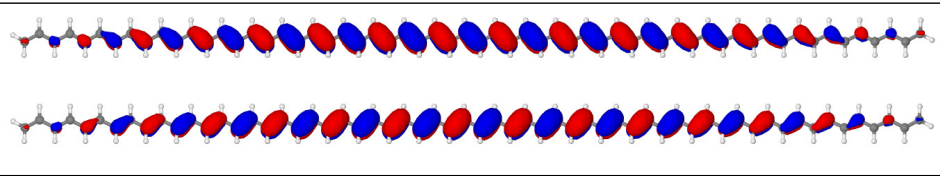 \\
\hline 1.000 & $\begin{array}{l}\mathrm{LUMO} \\
\mathrm{HOMO}\end{array}$ & 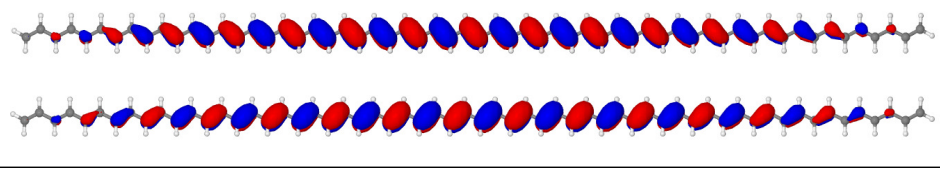 \\
\hline
\end{tabular}

de elétrons enquanto sua derivada possui uma descontinuidade em um número inteiro de elétrons. A ausência desta propriedade nos funcionais de troca e correlação LDA/GGA está relacionada ao SIE que, em geral, leva a falhas sistemáticas no cálculo dos gaps de energia. Seguindo essa vertente, e de acordo com a demonstração de SIE de Perdew, N. Sai e colaboradores [66 propuseram um esquema para ajustar o percentual de troca-exata no funcional híbrido PBEh de forma a obter a dependência linear esperada para a energia total em função do número de elétrons. Aplicando este esquema a poliacenos, os autores obtiveram um parâmetro ótimo de $\alpha=0,70$. Uma vez que este parâmetro é bastante similar ao ic- $\alpha$ extrapolado para a cadeia isolada de TPA, esperamos que nosso modelo baseado no critério de consistência interna resulte em uma redução considerável do SIE para sistemas $\pi$-estendidos.

Rigorosamente, lembramos que deveríamos nos ater à discussão apenas de estados ocupados, pois em princípio são essas as energias confiáveis nos modelos de campo médio. Já as energias de quasi-partícula seriam também confiáveis para os estados virtuais. Assim, analisamos ainda na tabela gráfica 3.3 a distribuição eletrônica do LUMO para a molécula $\mathrm{n}=30$, 
e observamos que este orbital apresenta uma simetria de conjugação $\pi$ e propriedades de localização bastante similares às do HOMO. Esta é uma característica comumente observada em polímeros conjugados. Portanto podemos esperar que o comportamento do gap eletrônico também siga a mesma tendência com o ajuste no parâmetro de troca-exata. De fato, analisando a evolução do LUMO como função da troca exata para os cálculos $\mathrm{G}_{0} \mathrm{~W}_{0} @ \mathrm{PBEh}$ verificamos que os valores de $\alpha$ que satisfazem a relação $\varepsilon_{K S-L U M O}=\varepsilon_{Q P-L U M O}$ são muito próximos aos ic- $\alpha$ obtidos no caso do HOMO. Por consequência, os gaps HOMO-LUMO calculados com o funcional ic-PBE (otimizado para o HOMO) são aproximadamente iguais aos resultados $\mathrm{G}_{0} \mathrm{~W}_{0}$. Isto pode ser visto nos diagramas de níveis de energia da Fig. 3.6 calculados para oligômeros de tamanhos intermediários.

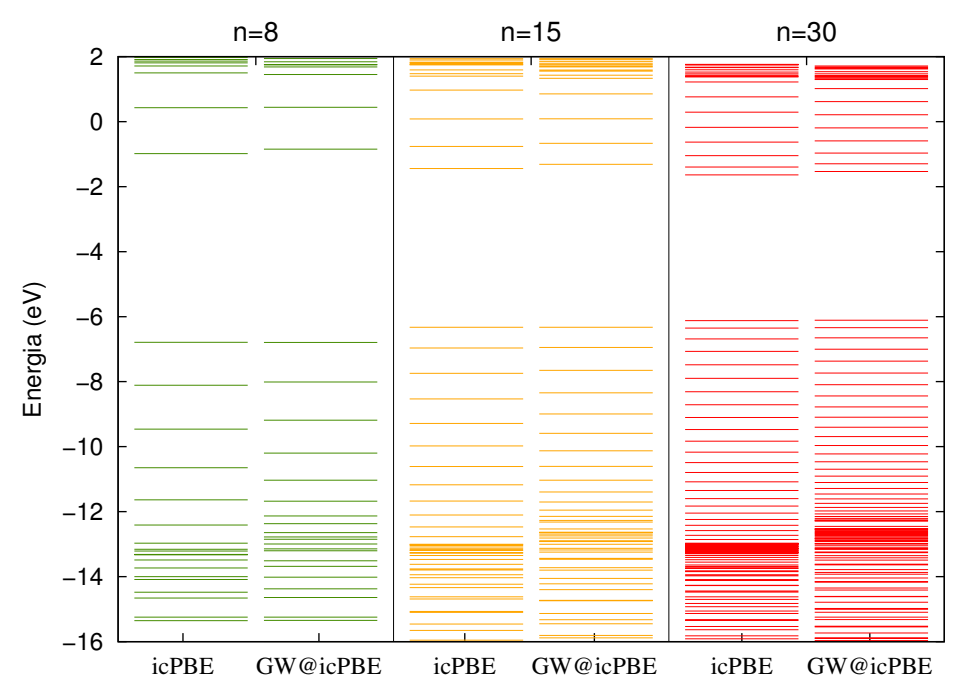

Figura 3.6: Espectro de níveis de energia calculados com os métodos icPBE e $G_{0} W_{0} @ i c P B E$ para oligoacetilenos de tamanho intermediário.

Fisicamente, o efeito de inclusão de troca-exata nos funcionais híbridos pode ser interpretado como uma blindagem média efetiva dos elétrons em um dado sistema, que no caso particular do modelo de consistência interna foi ajustado para o estado ocupado mais alto. No método GW, entretanto, os efeitos de muitos corpos levam a uma blindagem dinâmica dos elétrons, tal que a correção de quasipartícula aplicada aos orbitais KS originais depende da simetria específica desses estados [87]. Estas diferenças entre os modelos DFT e GW são visíveis na correção dos estados localizados de energias mais baixas ( $7 \mathrm{eV}$ abaixo do HOMO), conforme reportamos na Fig. 3.6. De fato, notamos que as energias de quasipartícula dos 
estados localizados obtidas após o cálculo $G_{0} W_{0} @ i c P B E$ estão cerca de 0,5 eV acima dos níveis DFTh localizados.

As correções para a posição dos níveis eletrônicos resultante dos cálculos GW comparando PBE e ic-PBE são mostradas na Fig. 3.7para três oligomeros de acetileno com diferentes tamanhos. A partir desse resultado observações importantes podem ser feitas. Primeiramente, comparando estado a estado como na Fig. 3.6 vemos que utilizando o funcional ic-PBE como ponto de partida, obtemos sempre correções muito menores que a partir do funcional PBE, tanto para os estados desocupados quanto para os ocupados, independente do tamanho dos oligômeros. Focalizando agora nas regiões onde temos estados localizados e deslocalizados, vemos que para ambos os funcionais PBE e icPBE a correção GW depende do caráter do estado, porém o valor da correção é sempre menor no caso do icPBE. 


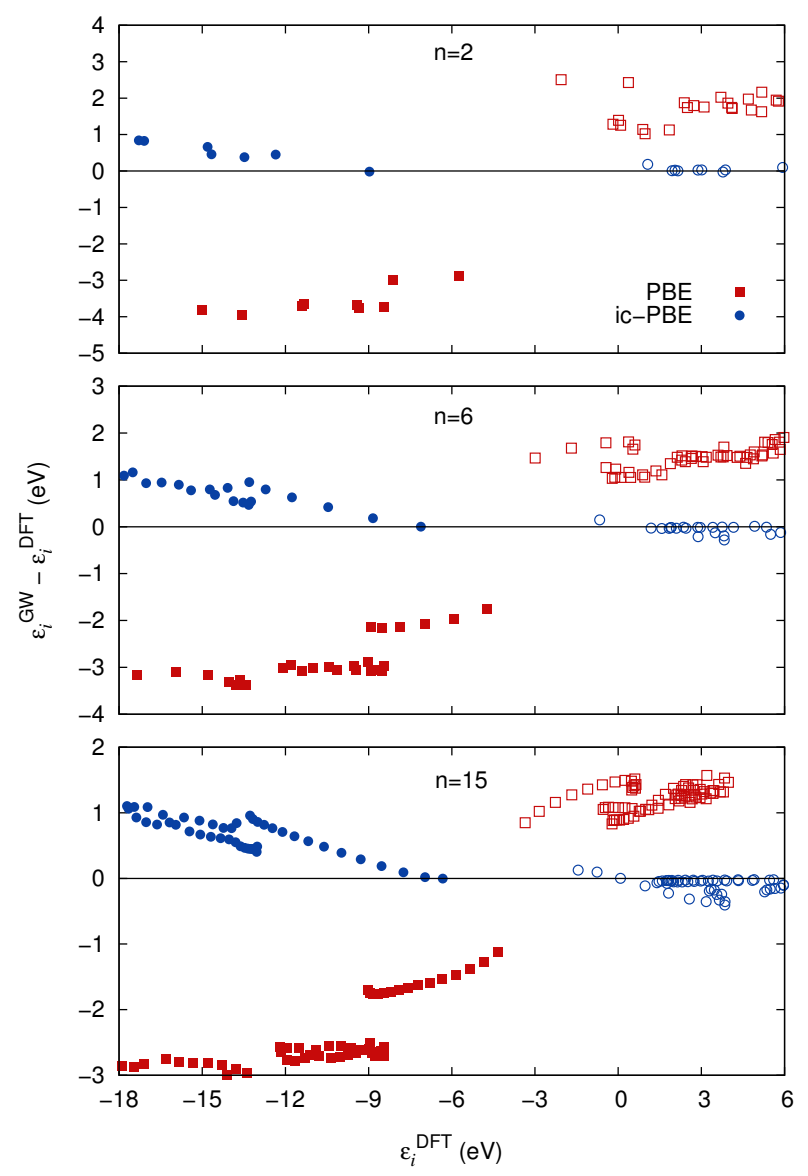

Figura 3.7: Valor das correções GW para os níveis de energia DFT como função do autovalor de energia DFT de cada estado eletrônico. Os símbolos preenchidos (vazios) correspondem aos estados ocupados (desocupados). Quadrados vermelhos representam orbitais de entrada PBE, e círculos em azul correspondem ao ic-PBE. 


\section{Capítulo 4}

\section{Funcional Híbrido Otimizado - OPBEh}

O estudo apresentado no capítulo anterior mostra que a fração ideal de troca exata inserida no funcional híbrido de modo a reproduzir, dentro de um modelo de campo médio, a energia de quasipartícula para o estado mais alto ocupado depende do comprimento molecular do sistema conjugado. Para tratar sistemas mais realísticos, como filmes de oligômeros ou polímeros que apresentem algum grau de desordem intramolecular, é necessário ter um único modelo de funcional que seja capaz de descrever a estrutura eletrônica do sistema amorfo com diferentes graus de localização dos orbitais moleculares de fronteira.

Neste capítulo definimos um funcional híbrido com uma fração constante (e independente do sistema) de troca exata, o qual denominamos OPBEh. Realizamos então um estudo sistemático para um conjunto de 15 moléculas orgânicas conjugadas em fase gasosa, que representam estruturas básicas de grande interesse para aplicações em dispositivos fotovoltáicos e eletrônica orgânica, no qual comprovamos a eficiência e precisão do funcional OPBEh no cálculo dos potenciais de ionização e gap fundamental agora através da comparação direta com os dados experimentais disponíveis. As moléculas selecionadas para este estudo incluem: acenos, oligofenilenos, oligofenileno-vinilenos (OPVs), fluoreno, alguns poliaromáticos 2D, e fulereno.

\subsection{Sistemas Conjugados 1D}

O esquema de consistência interna GW@PBEh utilizado no capítulo anterior para ajustar o parâmetro de troca-exata do funcional híbrido PBEh para a família de oligoacetilenos 
1D nos mostrou que o parâmetro ideal, ic- $\alpha$, de cada molécula é uma função do comprimento. Isto restringe, em princípio, a aplicação deste modelo a sistemas homogêneos, onde apenas um comprimento de conjugação específico está presente. Entretanto, em cálculos para situações mais realísticas, como por exemplo, para oligômeros ou polímeros em fase condensada, temos que considerar um regime no qual diferentes segmentos conjugados com diferentes graus de localização coexistem, alcançando em algumas regiões o limite onde podemos considerar o polímero infinito como um modelo razoável. Levando em conta esses aspectos morfológicos, desejamos encontrar um único parâmetro ótimo de troca-exata para o funcional híbrido PBEh que seja aplicável a sistemas conjugados não-homogêneos.

Como primeira aproximação, definimos a fração ótima de troca-exata como sendo o valor médio do parâmetro ic- $\alpha(1)$ obtido para as moléculas da família de oligoacetilenos. Assim, obtemos um valor ótimo de $\bar{\alpha}=0,80$, que coincide com o ic- $\alpha$ do oligômero OTA(6), e designamos o funcional correspondente por OPBEh. Na Fig. 4.1 mostramos os resultados para a energia do HOMO (IP) dos OTAs calculadas com funcionais baseados no potencial de troca-correlação PBE, utilizando diferentes frações de troca exata (de $\alpha=0$ a $\alpha=0,80$ ), incluindo novamente os resultados experimentais para cadeias curtas. A similaridade entre os valores obtidos com OPBEh e ic-PBE nos indica ser possível o uso de um único parâmetro de troca-exata para oligômeros de tamanhos variados.

Inicialmente, ainda tratando de acetilenos, selecionamos um derivado alquílico do oligômero com $n=7$ (ver Fig. 4.2) para o qual o espectro de fotoemissão foi medido em fase gasosa [71, e realizamos cálculos DFT e GW para avaliar a performance do funcional OPBEh. Neste caso, todas as coordenadas atômicas do oligômero foram previamente otimizadas no nível de teoria $\mathrm{PBE}+\mathrm{vdW} /$ tier2 para obtermos a geometria do estado fundamental. Verificamos que os grupos terminais tert-butil tem pouca influência sobre a geometria do OTA7, tal que a molécula mantém essencialmente a mesma estrutura planar e distâncias interatômicas do TPA puro e isolado.

A Fig. 4.3 mostra os espectros teóricos obtidos para a molécula OTA7 com diferentes modelos DFTh (PBE0 e OPBEh) e também com o método GW comparados à referência experimental de fotoemissão. Os auto-valores de energia DFTh e GW, artificialmente alargados com gaussianas de $0,25 \mathrm{eV}$, foram utilizados para simular o espectro com a mesma resolução experimental. Primeiramente, notamos que o OPBEh fornece a melhor descrição do espectro de fotoemissão experimental entre os métodos DFTh considerados com erros de $\Delta \mathrm{IP}_{1}=-0,22 \mathrm{eV}, \Delta \mathrm{IP}_{2}=0,28 \mathrm{eV}$ e $\Delta \mathrm{IP}_{3}=0,74 \mathrm{eV}$ para as três primeiras energias de ionização. 


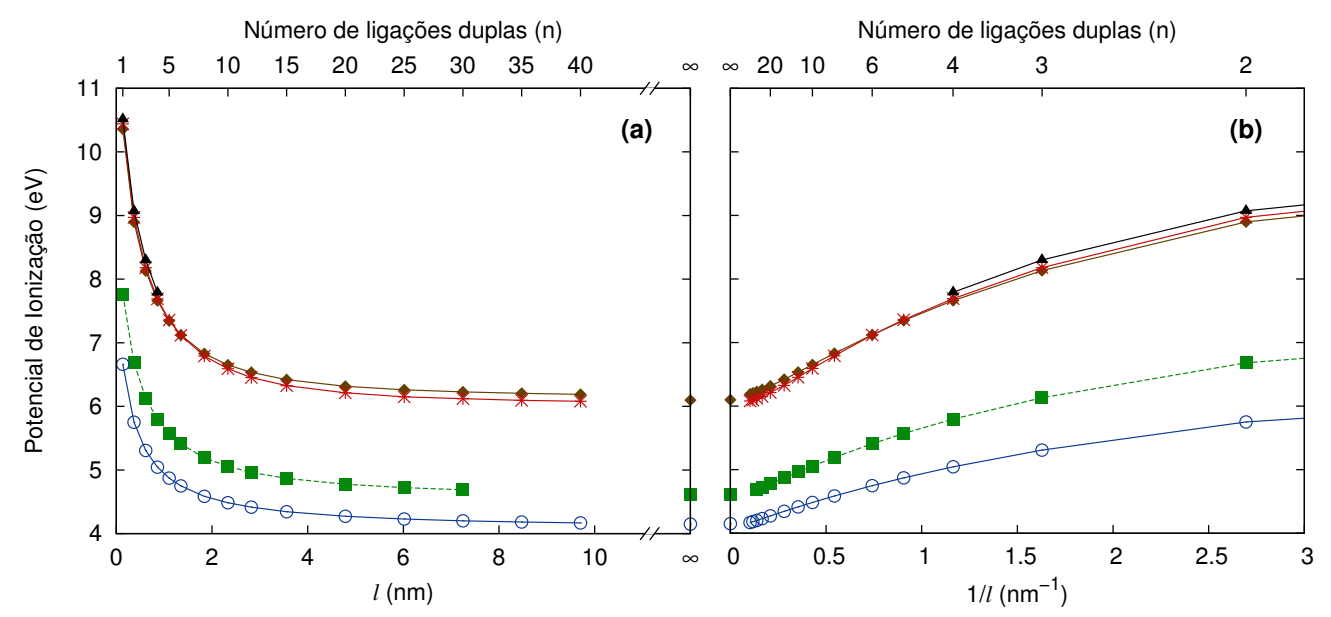

Figura 4.1: Evolução do potencial de ionização (IP) dos oligacetilenos como função do (a) comprimento e (b) inverso do comprimento das cadeias conjugadas. Os valores dos IPs foram calculados com os seguintes funcionais: PBE (círculos vazios), PBE0 (quadrados), ic-PBE (asteriscos) e OPBEh (losangos).

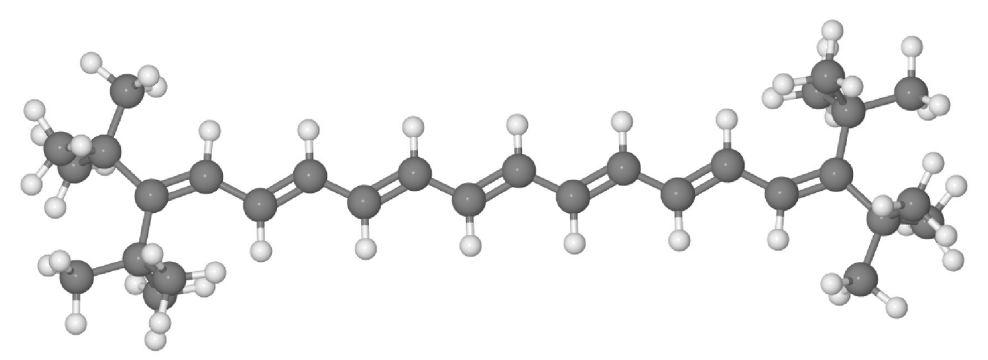

Figura 4.2: Estrutura otimizada do oligacetileno OTA7 com terminações alquílicas tert-butil.

O espectro obtido com PBE0 aparece deslocado para energias mais altas de modo que a primeira energia de ionização é subestimada em 1,89 eV. Portanto, vemos que a fração de troca-exata presente no funcional PBE0 é insuficiente para obter uma descrição razoável do espectro de fotoemissão.

Em geral, a correção GW aplicada aos níveis de energia DFTh resulta em um espectro de quasi-partícula em boa concordância com o experimento. Porém, devido à dependência do cálculo GW com a função de onda de partida DFTh, podemos esperar diferenças nos resultados finais para funcionais diferentes. Vemos que o resultado $\mathrm{G}_{0} \mathrm{~W}_{0} @ \mathrm{PBE} 0$ leva ainda 
a um erro considerável para a primeira energia de ionização, $\Delta \mathrm{IP}_{1}=0,71 \mathrm{eV}$, em relação ao valor experimental. Já o cálculo $\mathrm{G}_{0} \mathrm{~W}_{0} @ \mathrm{OPBEh}$ apresenta uma boa concordância com o espectro experimental tal que a diferença no primeiro pico é de apenas $0,24 \mathrm{eV}$, indicando que o funcional OPBEh fornece uma boa aproximação para os orbitais de quasi-partícula. Na região de energias mais baixas, as discrepâncias entre GW e OPBEh aumentam porém as características principais do espectro ainda são mantidas. Em suma, a consistência entre os métodos GW e OPBEh resulta numa boa estimativa para as duas primeiras energias de ionização experimentais e, além disso, também preserva as principais características do espectro experimental.

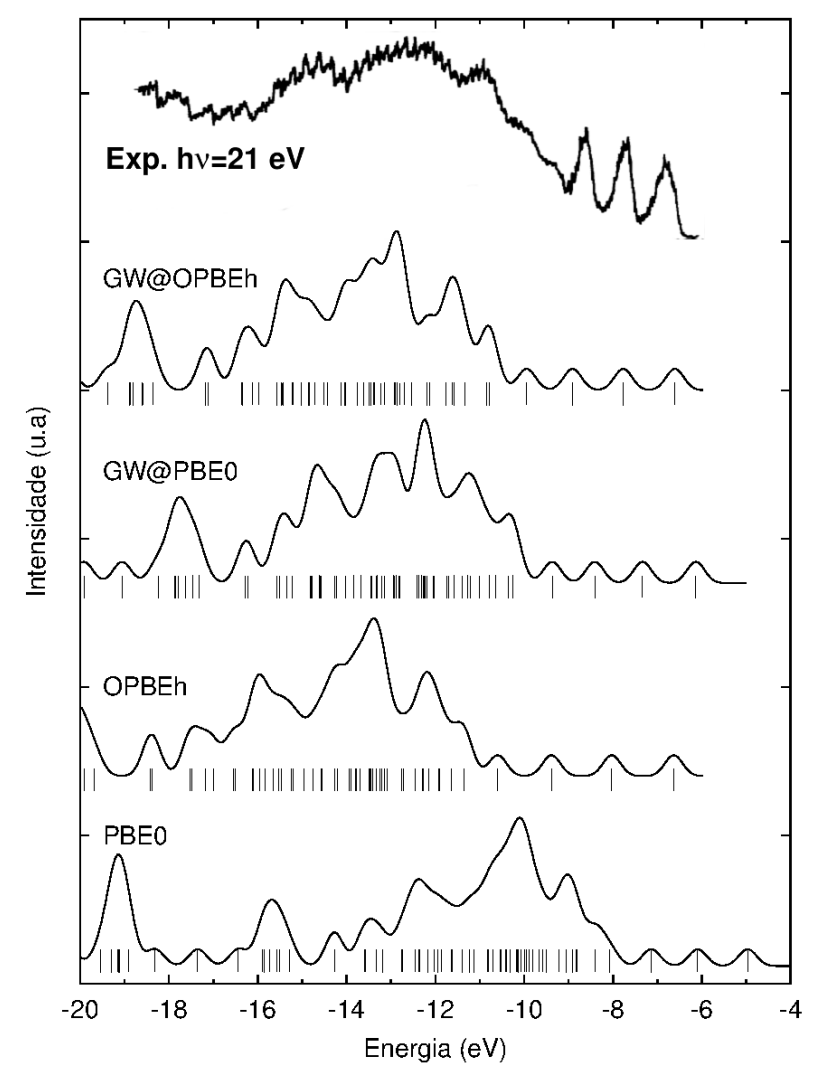

Figura 4.3: Espectro de níveis de energia DFT e QP do oligômero tb-OTA7 convoluídos com gaussianas de $0,25 \mathrm{eV}$, comparado com o espectro de fotoemissão experimental de fase gasosa 71 . 
Passamos a seguir ao estudo de moléculas conjugadas essencialmente diferentes dos acetilenos, com estruturas tipicamente 2D, e incluímos também o fulereno como exemplo de estrutura 3D.

\subsection{Outras Moléculas Orgânicas Conjugadas}

Inicialmente a geometria de equilíbrio DFT de cada uma das moléculas estudadas, mostradas na Fig. 4.4, foi determinada por meio de cálculos de relaxação estrutural utilizando o funcional $\mathrm{PBE}+\mathrm{vdW}$, adotando o critério de que a força máxima sobre cada átomo seja inferior a $5 \mathrm{meV} / \AA$. Em relação a densidade eletrônica e energia total, os critérios de convergência adotados foram de $10^{-6}$ elétrons e $10^{-5} \mathrm{eV}$, respectivamente. As funções de onda eletrônicas foram expandidas no conjunto de base numérico tight/tier 2. Os cálculos de otimização de geometria das moléculas isoladas resultam em estruturas essencialmente planares, exceto para os oligômeros de fenileno (Figs. 4.4i-k) onde observa-se um ângulo de torção entre anéis (a molécula de fulereno é intrisecamente 3D).

A tabela 4.1 lista os potenciais de ionização verticais, diretamente $\mathrm{IP}=-\varepsilon_{H O M O}$, das moléculas aqui tratadas calculados com diferentes formalismos de campo médio (DFT e HF). Os cálculos DFTh foram realizados com base no funcional de troca e correlação PBE considerando as diferentes frações de troca exata: PBE0 $(\alpha=0.25)$ e OPBEh $(\alpha=0.80)$. As energias de ionização PBE são claramente muito pequenas quando comparadas à referência experimental, com o erro chegando a $2,7 \mathrm{eV}$ para a molécula de quaterfenil. A inserção de uma pequena fração de troca exata no funcional PBE, conforme estabelecido no funcional padrão PBE0, diminui a energia do HOMO, melhorando ligeiramente o acordo com o experimento. Porém, a descrição dos IPs com o PBE0 é ainda bastante insatisfatória, com erros entre 0,98 eV (fulereno) e 1,93 eV (quaterfenil). Já o nosso modelo OPBEh resulta em muito melhor acordo com os dados experimentais. A precisão dos IPs obtidos com OPBEh é comparável aos cálculos HF, porém este último tende a subestimar o valor do IP para a maioria dos sistemas, exceto para a molécula de fulereno na qual o resultado HF fica 0,27 eV acima da referência experimental.

Uma comparação mais clara entre os diferentes modelos teóricos pode ser vista na Fig. 4.5 onde representamos graficamente os valores dos IPs teóricos versus experimentais para as 15 moléculas listadas na tabela 4.1. Vemos que os autovalores do HOMO obtidos com PBE e PBE0 subestimam o potencial de ionização das moléculas isoladas, enquanto nossos cálculos 


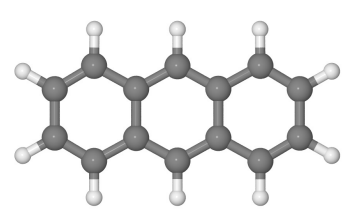

(a) Antraceno

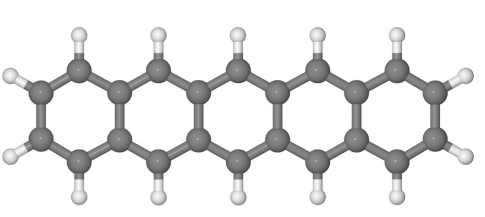

(b) Pentaceno

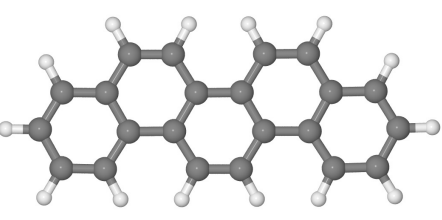

(c) Piceno

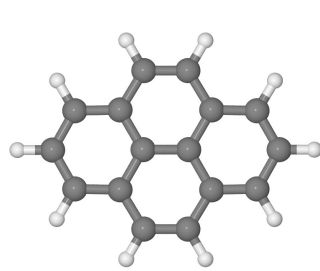

(d) Pireno

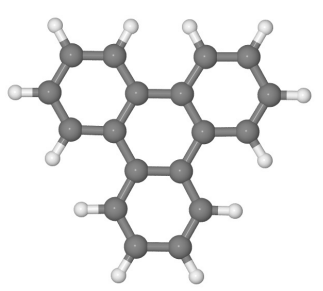

(e) Trifenileno

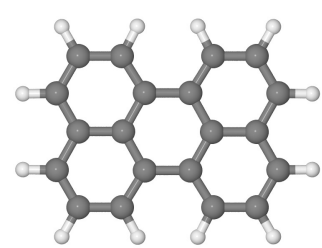

(f) Perileno

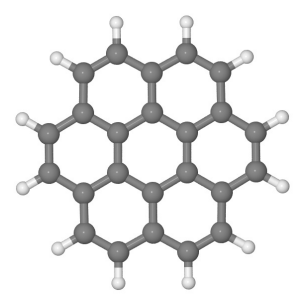

(g) Coroneno

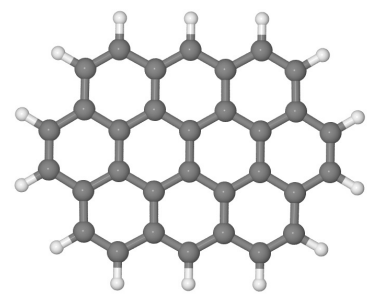

(h) Ovaleno

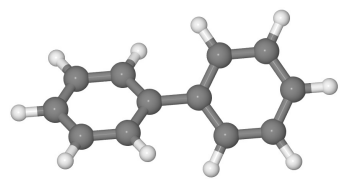

(i) Bifenil

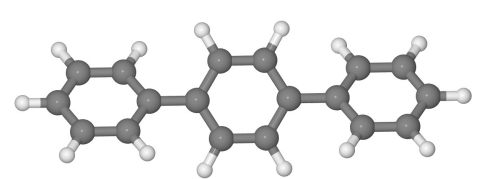

(j) Terfenil

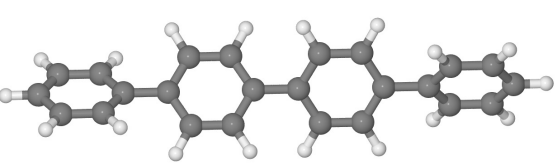

(k) Quaterfenil

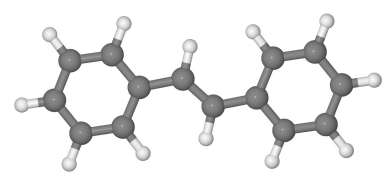

(1) Trans-estilbeno

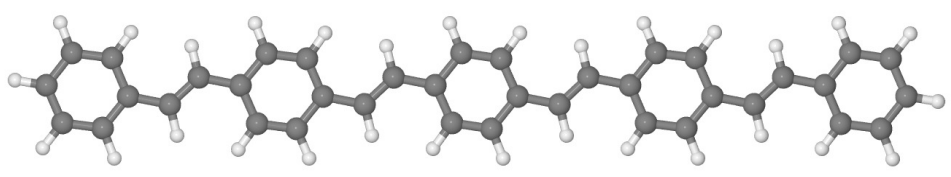

(m) P5V4

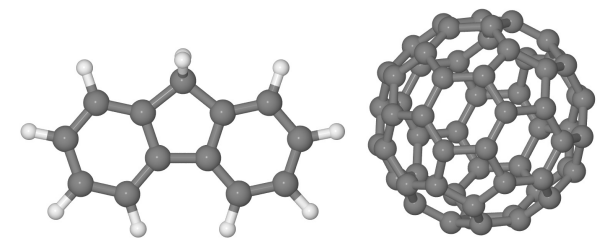

(n) Fluoreno

(o) Fulereno

Figura 4.4: Representação esquemática do conjunto de moléculas $\pi$-conjugadas selecionadas para estudo. 
Tabela 4.1: Potencial de ionização calculado como o negativo da energia do orbital mais alto ocupado - HOMO - utilizando diferentes formalismos teóricos (DFT e HF). Dados experimentais são incluidos para comparação.

\begin{tabular}{rcccccc}
\hline & & \multicolumn{5}{c}{ Potencial de Ionização (eV) } \\
\cline { 3 - 7 } molécula & fórmula & PBE & PBE0 & OPBEh & HF & Exp. ${ }^{*}$ \\
\hline Antraceno & $\mathrm{C}_{14} \mathrm{H}_{10}$ & 4,98 & 5,74 & 7,45 & 7,04 & $7,439 \pm 0,006$ \\
Pentaceno & $\mathrm{C}_{22} \mathrm{H}_{14}$ & 4,44 & 5,07 & 6,54 & 6,04 & $6,63 \pm 0,05$ \\
Piceno & $\mathrm{C}_{22} \mathrm{H}_{14}$ & 5,25 & 6,02 & 7,78 & 7,37 & $7,51 \pm 0,02$ \\
Pireno & $\mathrm{C}_{16} \mathrm{H}_{10}$ & 5,10 & 5,83 & 7,52 & 7,08 & $7,426 \pm 0,001$ \\
Perileno & $\mathrm{C}_{20} \mathrm{H}_{12}$ & 4,75 & 5,43 & 7,03 & 6,56 & $6,960 \pm 0,001$ \\
Trifenileno & $\mathrm{C}_{18} \mathrm{H}_{22}$ & 5,58 & 6,39 & 8,22 & 7,81 & $7,87 \pm 0,02$ \\
Coroneno & $\mathrm{C}_{24} \mathrm{H}_{12}$ & 5,24 & 5,95 & 7,56 & 7,07 & $7,29 \pm 0,03$ \\
Ovaleno & $\mathrm{C}_{32} \mathrm{H}_{14}$ & 4,80 & 5,41 & 6,85 & 6,30 & $6,76 \pm 0,01$ \\
Trans-estilbeno & $\mathrm{C}_{14} \mathrm{H}_{12}$ & 5,22 & 6,02 & 7,88 & 7,52 & $7,89 \pm 0,05$ \\
P5V4 & $\mathrm{C}_{38} \mathrm{H}_{30}$ & 4,71 & 5,38 & 7,05 & 6,61 & 6,7 \\
Bifenil & $\mathrm{C}_{12} \mathrm{H}_{10}$ & 5,73 & 6,61 & 8,57 & 8,22 & $8,16 \pm 0,13$ \\
Terfenil & $\mathrm{C}_{18} \mathrm{H}_{14}$ & 5,49 & 6,32 & 8,19 & 7,81 & $7,80 \pm 0,03$ \\
Quaterfenil & $\mathrm{C}_{24} \mathrm{H}_{18}$ & 5,34 & 6,15 & 7,98 & 7,57 & $8,08 \pm 0,05$ \\
Fluoreno & $\mathrm{C}_{13} \mathrm{H}_{10}$ & 5,45 & 6,29 & 8,17 & 7,79 & $7,91 \pm 0,02$ \\
Fulereno & $\mathrm{C}_{60}$ & 5,82 & 6,59 & 8,31 & 7,84 & $7,57 \pm 0,01$ \\
\hline
\end{tabular}

* Os dados experimentais de fase gasosa foram obtidos da base de dados do NIST - http://webbook.nist.gov/chemistry/.

mostram que os resultados HF são significativamente mais próximos do experimento, confirmando que a inclusão de $25 \%$ de troca exata incluidos no PBE0 não é suficiente. Já a fração de $80 \%$ de troca exata do funcional OPBEh, definida com base no procedimento internamente consistente GW@PBEh, resulta em IPs em muito bom acordo com o experimento, inclusive com erros menores que o HF. Entretanto, conforme demonstrado no capítulo anterior, a quantidade ideal de troca exata a ser utilizada em um funional híbrido de forma a obter a melhor descrição possível para as energias de quasipartícula é dependente do sistema, o que justifica a dispersão de valores do OPBEh em torno da reta $\mathrm{IP}_{\text {teor }}=\mathrm{IP}_{\text {exp }}$ da Fig. 4.5 .

Analisando em detalhes o desvio no IP para cada molécula da tabela 4.1 podemos identificar alguns aspectos importantes quanto à influência da troca exata. Primeiramente, notamos que, além da estrutura molecular quasi-1D, os oligoacenos possuem um caráter de conjugação $\pi$ bastante similar aos oligoacetilenos para o qual o funcional OPBEh foi proje- 


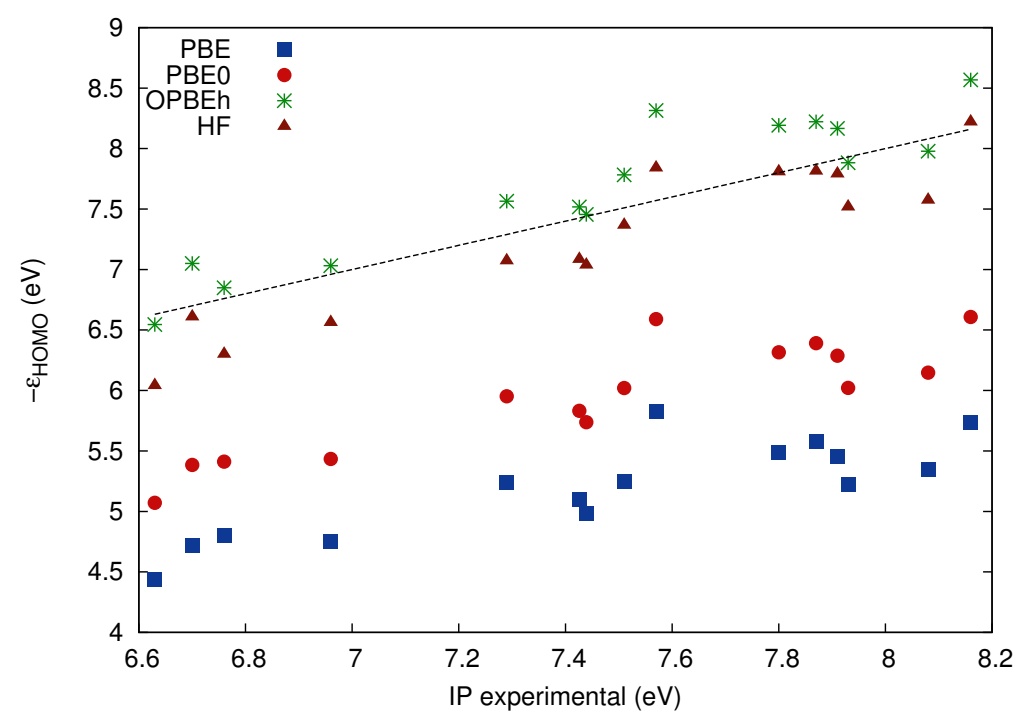

Figura 4.5: Comparação dos potenciais de ionização teóricos, calculados como negativo da energia do KS-HOMO, e experimentais. A linha tracejada corresponde a reta $y=x$ no qual os valores teóricos e experimentais coincidem. Os valores numéricos são listados na tabela 4.1 .

tado. Do ponto de vista estrutural, os oligoacenos podem ser vistos como duas cadeias de poliacetileno acopladas lateralmente. De fato, analisando a tabela gráfica 4.2 vemos que os orbitais de fronteira HOMO e LUMO apresentam o mesmo caráter de localização espacial observada anteriormente para os OTAs. Consequentemente, o funcional OPBEh leva a excelente resultados para os IPs do antraceno e pentaceno, com um dos menores desvios em relação ao experimento entre o conjunto de moléculas estudadas.

Tabela 4.2: Densidade eletrônica dos orbitais moleculares de fronteira, LUMO e HOMO, calculada para a molécula de pentaceno utilizando os funcionais PBE e OPBEh.

orbitais


Outra classe de moléculas que preserva o caráter $\pi$ conjugado dos OTAs são os oligomeros de fenileno-vinileno, representados nesse estudo pelo trans-estilbeno e P5V4. Para o trans-estilbeno, o IP obtido com nosso modelo OPBEh é apenas $0,05 \mathrm{eV}$ menor que o valor experimental, indicando que a fração de troca exata ótima de $80 \%$ é bastante eficiente para descrever esse tipo de sistema. No caso da molécula de P5V4, o desvio em relação ao experimento aumenta para $0,35 \mathrm{eV}$. Entretanto, devemos lembrar que o ic- $\alpha$ diminui exponencialmente com o comprimento molecular, tal que para cadeias mais longas, a fração constante do OPBEh passa a ser menos eficiente. Isso justifica o fato de o OPBEh superestimar o IP da molécula de P5V4.

A eficiência do modelo OPBEh está também relacionada ao grau de localização dos estados de fronteira, como pode ser observado para a série de oligofenilenos da tabela 4.1. Por exemplo, no caso do bifenil, a geometria de equilibrio DFT $+\mathrm{vdW}$ apresenta um ângulo de torção entre os anéis fenil de 39,7 $7^{\circ}$ que contribui para uma maior localização dos estados HOMO e LUMO da molécula. Devido a este efeito de localização, o funcional OPBEh preve um IP para o bifenil que é $0,41 \mathrm{eV}$ maior que o valor experimental, portanto com um desvio bem maior do que o obtido para a molécula conjugada de tamanho equivalente, trans-estilbeno. Para o oligomero com três anéis, terfenil, notamos que o ângulo de torção médio entre anéis diminui em $1^{\circ}$, tal que o erro obtido no cálculo do IP com OPBEh é ligeiramente menor $(0,39 \mathrm{eV})$ que no caso do bifenil. Considerando o próximo oligomero da série, quaterfenil, a tendência no cálculo do IP se confirma, ou seja, com uma torção média de $38,4^{\circ}$ o erro absoluto em relação ao experimento passa a ser de $0,10 \mathrm{eV}$.

Além das moléculas 1D lineares, analisamos também as propriedades eletrônicas de sistemas conjugados de caráter 2D ou quasi-2D. Estas moléculas podem ser vistas como nanoestruturas de carbono derivadas do grafeno, com geometrias planares e delocalização dos orbitais de fronteira no plano bidimensional. Entre as moléculas analisadas, destacamos o piceno por ter o mesmo número de átomos que o pentaceno porém numa configuração tipo armchair que lhe confere uma geometria quasi-2D com propriedades eletrônicas bastante distintas. De fato, apenas com uma mudança estrutural, o IP experimental aumenta de 6,63 eV para 7,51 eV quando passamos do pentaceno ao piceno, enquanto o erro absoluto no cálculo do IP obtido com o OPBEh aumenta de $0,09 \mathrm{eV}$ para $0,27 \mathrm{eV}$. No caso do piceno, o método HF fornece a melhor descrição para o IP entre os modelos de campo médio analisados. Uma possível explicação para este resultado está na relação entre o gap elétrico e a blindagem eletrônica, ou seja, quanto maior o gap menor a blindagem, e portanto maior 
deve ser a fração de troca exata para descrever corretamente os níveis de fronteira. Uma vez que o gap experimental, $\mathrm{E}_{g a p}=\mathrm{IP}-\mathrm{EA}$, do piceno é aproximadamente 1,7 eV maior que o do pentaceno (ver tabela 4.2) espera-se que a fração de troca exata ótima com respeito as energias de quasi-partícula seja ainda mais próxima do limite HF, portanto maior que o valor de $80 \%$ definido no OPBEh.

Em comparação a cálculos GW da literatura, o nosso modelo OPBEh fornece uma boa descrição dos IPs. Por exemplo, nos cálculos $\mathrm{G}_{0} \mathrm{~W}_{0} @ \mathrm{HF}$ realizados por Blase e colaboradores 88 os IPs obtidos para as moléculas de antraceno, pentaceno, fluoreno e C60 foram 7,03 eV, 6,08 eV , 7,64 eV e 7,41 eV respectivamente, enquanto nossos cálculos com OPBEh resultaram em 7,04 eV, 6,54 eV, 8,17 eV e 7,84 eV. Assim, vemos que o nível de precisão do nosso modelo OPBEh no cálculo dos IPs é comparável ao de um cálculo de muitos corpos na aproximação $\mathrm{G}_{0} \mathrm{~W}_{0}$.

Tabela 4.3: Gap fundamental do conjunto de moléculas $\pi$-conjugadas estendidas calculado como a diferença de energia HOMO-LUMO utilizando diferentes formalismos teóricos (DFT e HF). Dados experimentais são incluidos para comparação.

\begin{tabular}{rlccccc}
\hline & & \multicolumn{5}{c}{ Gap Fundamental (eV) } \\
\cline { 3 - 7 } molécula & fórmula & PBE & PBE0 & OPBEh & HF & Exp. $^{*}$ \\
\hline Antraceno & $\mathrm{C}_{14} \mathrm{H}_{10}$ & 2,32 & 3,80 & 7,13 & 8.33 & 6,91 \\
Pentaceno & $\mathrm{C}_{22} \mathrm{H}_{14}$ & 1,14 & 2,37 & 5,21 & 6.28 & 5,24 \\
Piceno & $\mathrm{C}_{22} \mathrm{H}_{14}$ & 2,95 & 4,47 & 7,89 & 9.08 & 6,97 \\
Pireno & $\mathrm{C}_{16} \mathrm{H}_{10}$ & 2,63 & 4,06 & 7,33 & 8.51 & 7,02 \\
Perileno & $\mathrm{C}_{20} \mathrm{H}_{12}$ & 1,87 & 3,20 & 6,28 & 7.39 & 5,99 \\
Trifenileno & $\mathrm{C}_{18} \mathrm{H}_{22}$ & 3,56 & 5,15 & 8,67 & 9.89 & 7,58 \\
Coroneno & $\mathrm{C}_{24} \mathrm{H}_{12}$ & 2,87 & 4,26 & 7,37 & 8.48 & 6,82 \\
Ovaleno & $\mathrm{C}_{32} \mathrm{H}_{14}$ & 1,93 & 3,12 & 5,87 & 6.89 & - \\
Trans-estilbeno & $\mathrm{C}_{14} \mathrm{H}_{12}$ & 2,76 & 4,32 & 7,89 & 9.14 & 7,50 \\
P5V4 & $\mathrm{C}_{38} \mathrm{H}_{30}$ & 1,66 & 2,95 & 6,13 & 7.30 & - \\
Bifenil & $\mathrm{C}_{12} \mathrm{H}_{10}$ & 3,91 & 5,61 & 9,36 & 10.36 & 8,03 \\
Terfenil & $\mathrm{C}_{18} \mathrm{H}_{14}$ & 3,38 & 4,99 & 8,57 & 9.78 & - \\
Quaterfenil & $\mathrm{C}_{24} \mathrm{H}_{18}$ & 3,11 & 4,67 & 8,16 & 9.35 & 7,42 \\
Fluoreno & $\mathrm{C}_{13} \mathrm{H}_{10}$ & 3,61 & 5,24 & 8,85 & 9.95 & 7,63 \\
Fulereno & $\mathrm{C}_{60}$ & 1,65 & 2,94 & 5,87 & 6.95 & 4,89 \\
\hline
\end{tabular}

* Os gaps experimentais de fase gasosa foram determinados utilizando a definiçao IP-EA com valores obtidos da base de dados do NIST - http://webbook.nist.gov/chemistry/. 
Os valores do gap fundamental das moléculas em fase gasosa calculados como a diferença de energia HOMO-LUMO utilizando diferentes modelos são listados na tabela 4.3. Os gaps experimentais definidos como IP-EA são também incluídos na tabela 4.3 para comparação. Primeiramente, notamos que o funcional PBE subestima fortemente o gap elétrico das moléculas isoladas. A inclusão de troca exata na DFT tende a aumentar o gap, porém os valores obtidos com o funcional PBE0 são ainda muito pequenos, ficando em média 2,61 eV abaixo da referência experimental. Por outro lado, o método HF sistematicamente superestima os gaps de energia, como esperado, com o erro chegando a 2,33 eV para a molécula de bifenil e com o menor erro de 1,04 eV para o pentaceno. Estes erros são bastante reduzidos com o uso do funcional híbrido otimizado OPBEh. De fato, o OPBEh apresenta o melhor acordo com o experimento no cálculo do gap fundamental entre os modelos de campo médio considerados. Portanto, concluímos que o OPBEh fornece uma boa descrição não somente para o HOMO, mas também para o primeiro estado virtual, LUMO.

A mudança no parâmetro de troca exata afeta significativamente distribuição eletrônica total, conforme exemplificado na Fig. 4.6 para as moléculas de coroneno e P5V4. A diferença na densidade eletrônica OPBEh-PBE indica que a troca exata favorece um acúmulo de cargas em torno de cada átomo do sistema (região em vermelho), enquanto que a distribuição eletrônica em torno da ligação entre os átomos é reduzida. Esse efeito de aumento na localização da densidade eletrônica com o funcional OPBEh pode ser entendido como uma consequência do cancelamento parcial da auto-interação devido a inclusão de troca exata.

A superioridade do funcional OPBEh na obtenção dos IPs e gaps elétricos é evidenciada pelo histograma da Fig. 4.7, que representa o erro médio absoluto (MAE) no cálculos dessas propriedades para as 15 moléculas selecionadas. Como é bem conhecido, os funcionais DFT semi-locais, tal como PBE, levam a uma descrição muito ruim para os níveis de fronteira HOMO e LUMO, resultando em um MAE da ordem de 2,3 eV e 4,1 eV no cálculo dos IPs e gaps, respectivamente. O funcional híbrido PBE0 melhora o resultado PBE em até $33 \%$ no caso do IP e em 36\% para o gap, porém os erros são ainda muito grandes quando comparados ao método HF. Este último fornece uma boa descrição para o IP com base na aproximação de Koopmans, entretanto a falta de correlação eleva os níveis virtuais para energias mais altas, resultando em gaps 1,8 eV maiores que o experimento. Entre os modelos de campo médio avaliados, destacamos que o funcional OPBEh produz IPs e gaps com os menores desvios em relação aos valores experimentais. Embora a precisão do OPBEh seja comparável ao HF 


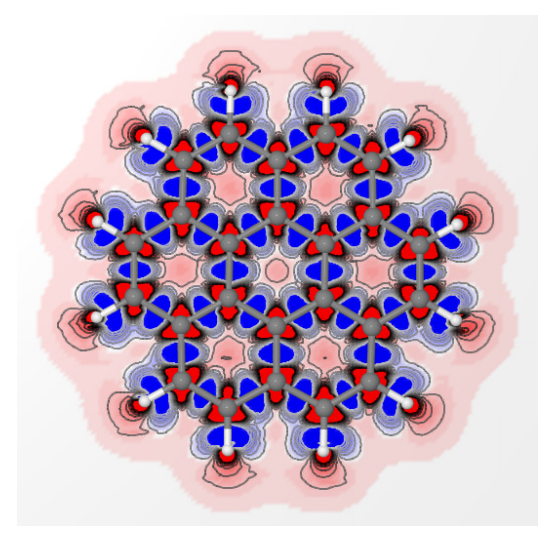

(a) Coroneno

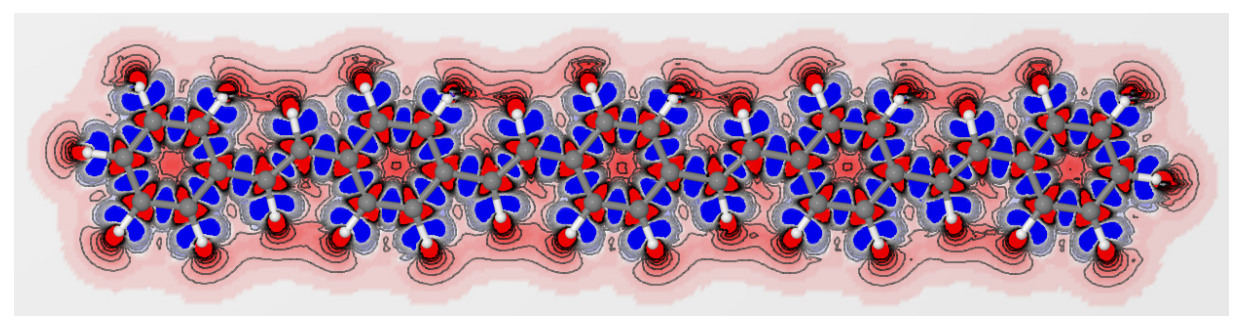

(b) P5V4

Figura 4.6: Diferença da densidade eletrônica total calculada com OPBEh e PBE para as moléculas de coroneno e P5V4.

para o cálculo dos IPs, o erro médio do OPBEh no cálculo dos gaps é cerca de $62 \%$ menor que o HF.

\subsection{Moléculas Orgânicas Alquiladas}

Em diversos sistemas poliméricos ou mesmo moleculares utilizados em dispositivos é bastante comum o uso de derivações alquílicas (cadeias de carbono saturadas) quimicamente ligadas ao segmento conjugado, cuja principal função é facilitar a solubilização desses sistemas. Esses subsistemas que constituem o polímero como um todo possuem propriedades eletrônicas bastante distintas. Em geral, os níveis de fronteira HOMO e LUMO que definem as propriedades eletrônicas e óticas do polímero pertencem ao segmento conjugado, enquanto que os estados pertencentes as cadeias alquílicas estão em uma região de mais baixa energia que, em princípio, não influenciam diretamente as propriedades do polímero. Entretanto, a troca exata utilizada nos funcionais híbridos pode ter efeitos diferentes sobre os níveis de 


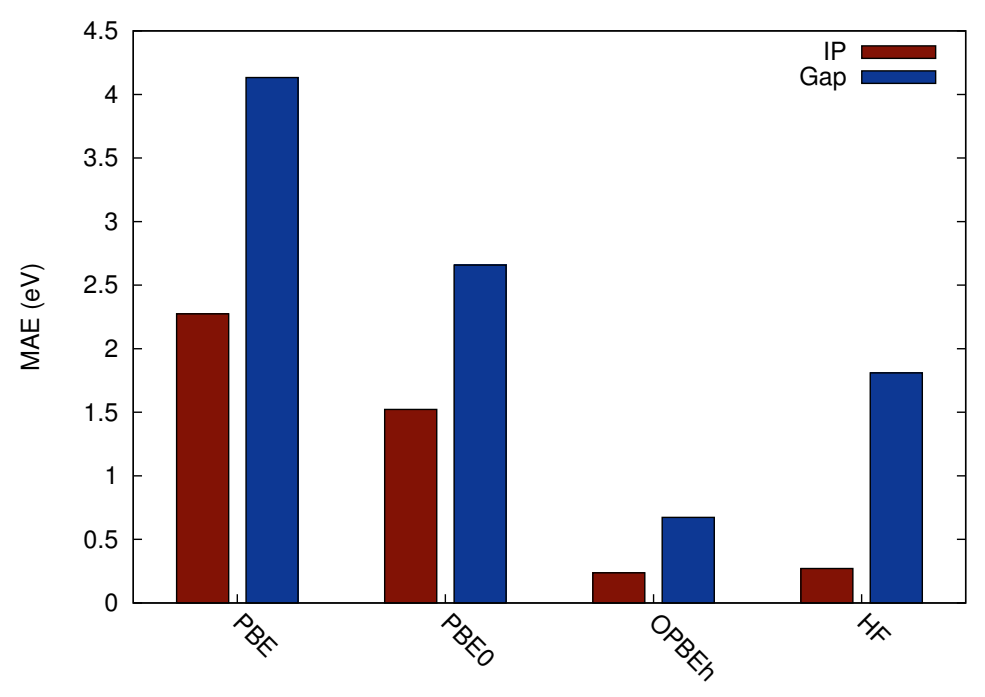

Figura 4.7: Erro médio absoluto (MAE) dos IPs e Gaps HOMO-LUMO calculados com diferentes modelos teóricos para o conjunto de moléculas conjugadas da Fig. 4.4. Em todos os casos, o MAE é determinado com respeito aos valores experimentais.

fronteira dos sistemas conjugados e saturados. Assim, é importante analisar se o funcional OPBEh é capaz de descrever corretamente a estrutura eletrônica de sistemas moleculares híbridos constituidos de um segmento conjugado com derivações alquílicas.

Como sistema modelo, selecionamos a molécula de fluoreno (ver Fig. 4.4(n) e adicionamos dois grupos alquílicos propril perpendicularmente ao eixo conjugado, conforme mostrado na Fig. 4.8. Para esta molécula, realizamos primeiramente um cálculo de otimização de geometria seguindo o mesmo protocolo computacional já descrito na seção anterior. Em seguida, calculamos a estrutura eletrônica da molécula híbrida, na geometria de equilíbrio DFT, utilizando os funcionais PBE e OPBEh, e comparando com os resultados obtidos via cálculos $\mathrm{G}_{0} \mathrm{~W}_{0}$ iniciados a partir das diferentes densidades eletrônicas DFT.

A tabela 4.4 lista os valores obtidos com dois diferentes formalismos teóricos para os níveis de fronteira HOMO e LUMO juntamente com os dados experimentais disponíveis. Primeiramente, no caso da molécula de fluoreno, notamos que o funcional PBE padrão superestima [subestima] a energia do HOMO [LUMO] tal que a correção GW é fundamental para um bom acordo com os valores experimentais. Já as energias do HOMO e LUMO obtidas com o funcional OPBEh fornecem uma boa aproximação tanto para o IP quanto para o EA, com um erro máximo de $0,4 \mathrm{eV}$ em relação ao experimento. 


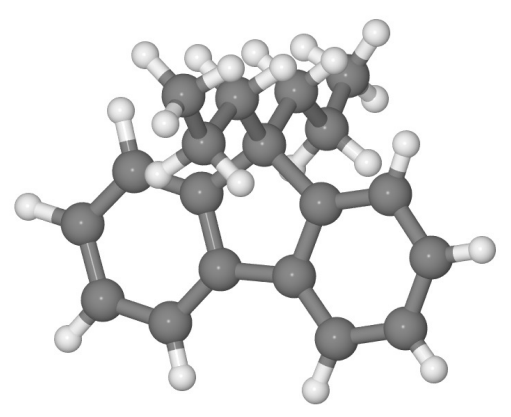

Figura 4.8: Representação esquemática da molécula de propil-fluoreno.

Tabela 4.4: Energias dos orbitais moleculares de fronteira (em eV) calculados com diferentes formalismos teóricos para as moléculas de fluoreno, e para o derivado alquílico, propilfluoreno. As correções GW para cada estado são também indicadas na tabela. Os valores experimentais disponíveis são incluidos para comparação.

\begin{tabular}{rccccc}
\hline \multicolumn{5}{c}{ fluoreno } \\
\hline PUMO & $-1,84$ & 0,09 & 0,68 & 0,72 & $0,28 \pm 0,03$ (EA) \\
HOMO & $-5,45$ & $-7,53$ & $-8,17$ & $-7,98$ & $7,91 \pm 0,02$ (IP) \\
\hline \multicolumn{5}{c}{ propil-fluoreno } \\
\hline LUMO & $-1,88$ & $-0,09$ & 0,63 & 0,57 & - \\
HOMO & $-5,42$ & $-7,37$ & $-8,13$ & $-7,87$ & - \\
Corr. & & -1.95 & & 0,26 & - \\
\hline HOMO-4 & $-7,43$ & $-10,24$ & $-11,82$ & $-11,15$ & \\
Corr. & & -2.81 & & 0,67 & -
\end{tabular}

* Os dados experimentais de fase gasosa foram obtidos da base de dados do NIST - http://webbook.nist.gov/chemistry/.

Dentro da aproximação DFT, a influência das derivações alquílicas propil sobre os autovalores dos níveis HOMO e LUMO são desprezíveis, tal que estes níveis variam por menos de 0,05 eV comparado a molécula pristina. Já os cálculos realizados na aproximação GW são mais sensíveis a presença dos grupos alquílicos, mas a energia QP-HOMO aumenta também pouco $(0,16 \mathrm{eV})$ com a inserção dos grupos propil. De interesse aqui, os resultados obtidos com os métodos OPBEh e GW@OPBEh apresentam muito bom acordo tanto para os níveis de fronteira, HOMO e LUMO, quanto para os estados mais profundos, como o HOMO-4, localizados sobre as alquilas. Através desse exemplo, vemos que o modelo OPBEh pode ser 
bastante eficiente e preciso para o cálculo das propriedades eletrônicas de sistemas moleculares híbridos que incluem subgrupos com diferentes características eletrônicas.

\subsection{Aplicação do OPBEh a Interfaces Orgânicas}

Em geral, os dipositivos orgânicos são construídos em uma estrutura vertical de multicamadas no qual a camada ativa é constituída por moléculas ou polímeros de caráter eletrônico doador e aceitador [89]. Nesses dispositivos, a recombinação ou separação elétron-buraco ocorrem na região interfacial entre as camadas transportadoras de elétrons e de buracos. Em particular, a posição do HOMO pertencente ao doador orgânico relativa ao LUMO do aceitador está diretamente relacionada a barreira de energia para o transporte de carga entre os dois sistemas. Portanto, o conhecimento detalhado do alinhamento dos níveis de energia em heterojunções orgânico/orgânico é essencial para o controle e otimização dos dispositivos orgânicos.

Nesta seção, analisamos o efeito do parâmetro de troca exata $\alpha$ na determinação do alinhamento entre os níveis eletrônicos de fronteira de interfaces orgânico/orgânico. Como primeiro exemplo, discutimos o alinhamento de níveis entre as moléculas de P5V4 e C60 no caso não-interagente, isto é, no limite Schottky-Mott. Estas moléculas constituem um par doador-aceitador de elétrons, de forma que após a formação da interface, uma camada de dipolo pode existir entre os dois sistemas, influenciando o alinhamento mútuo dos níveis de energia.

A Fig. 4.9 mostra o diagrama de níveis de energia calculado para as moléculas de P5V4 e C60 isoladas, ilustrando o alinhamento previsto no caso não-interagente, ou seja, considerando uma separação infinita entre os dois sistemas. Os espectros de autovalores foram obtidos com os funcionais PBE e OPBEh utilizando o conjunto de base tier 2, e são comparados com os valores experimentais disponíveis. Neste exemplo simples, os funcionais PBE e OPBEh concordam quanto a descrição qualitativa do alinhamento de níveis, isto é, nenhuma transferência de carga deve ocorrer entre os sistemas na ausência de campos externos, uma vez que o HOMO do doador (P5V4) está abaixo do LUMO do aceitador (C60) em ambos os modelos. Entretanto, uma vez que a transferência de carga deve ocorrer do orbital HOMO do doador (D) para o LUMO do aceitador (A), vemos que o funcional PBE subestima a barreira de energia em aproximadamente $3,5 \mathrm{eV}$ devido aos erros no cálculo dos IPs e gaps elétricos já discutidos na seção 4.1. Já o funcional OPBEh prevê uma barreira de energia 
$\mathrm{HOMO}_{D}-\mathrm{LUMO}_{A}$ de 4,6 eV, concordando com o valor experimental de 4,0 eV estimado no limite Schottky-Mott. Estudos experimentais [6] indicam a formação de um campo de dipolo na interface P5V4/C60 que influencia no alinhamento dos níveis de energia entre os dois sistemas.

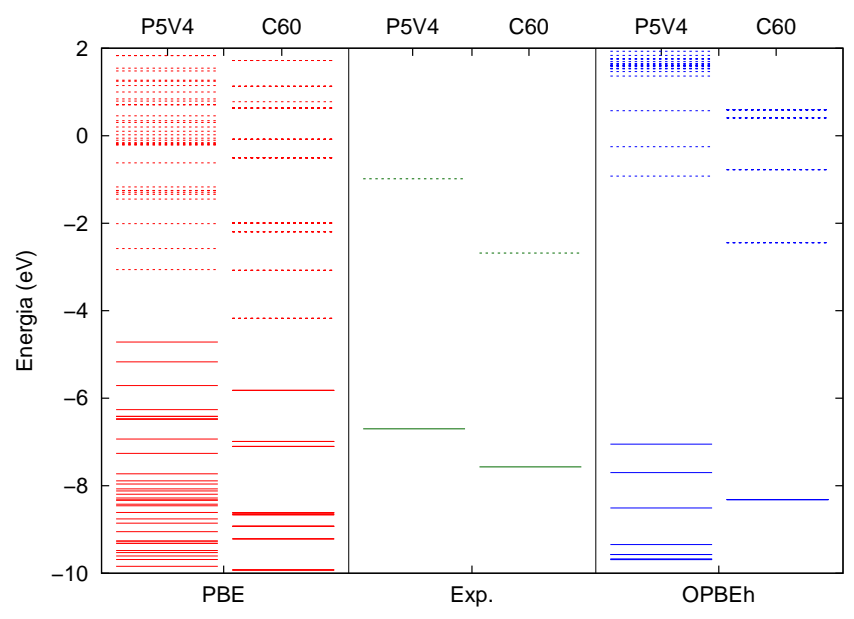

Figura 4.9: Diagrama de alinhamento dos níveis de energia calculado com diferentes métodos teóricos para as moléculas de P5V4 e C60 no caso não-interagente, ou seja, considerando uma separação infinita. Os valores experimentais dos IPs e EAs são incluidos para comparação. As linhas tracejadas indicam os níveis de energia dos estados virtuais.

Em seguida, consideramos como segundo exemplo a interface pentaceno/C60 para o qual realizamos um estudo teórico da estrutura eletrônica e alinhamento dos níveis de energia tanto no caso dos sistemas isolados quanto no caso interagente. Como primeira aproximação, simulamos a interação entre os dois sistemas na forma de um dímero, isto é, uma molécula de pentaceno é aproximada à uma molécula de C60 no vácuo.

Primeiramente, analisamos o problema do alinhamento dos níveis de energia na interface pentaceno/C60 para o caso não-interagente. O diagrama de níveis de energia obtido com os funcionais PBE e OPBEh para cada uma das moléculas em fase gasosa é mostrado na Fig. 4.10, onde incluímos também os dados experimentais disponíveis para comparação. Assim como no exemplo anterior, embora os funcionais PBE e OPBEh concordem qualitativamente quanto à posição relativa dos níveis de energia do doador e aceitador, o PBE subestima severamente a barreira de energia $\mathrm{HOMO}_{D}-\mathrm{LUMO}_{A}$, resultando em um valor de $\sim 0,5$ eV. Já com o OPBEh obtemos uma descrição muito melhor para os níveis de fronteira tanto do pentaceno quanto do C60, de forma que a barreira $\mathrm{HOMO}_{D^{-}} \mathrm{LUMO}_{A}$ de $4,1 \mathrm{eV}$ 
está em excelente acordo com a estimativa experimental de $3,9 \mathrm{eV}$ baseada no potencial de ionização e afinidade eletrônica das moléculas isoladas.

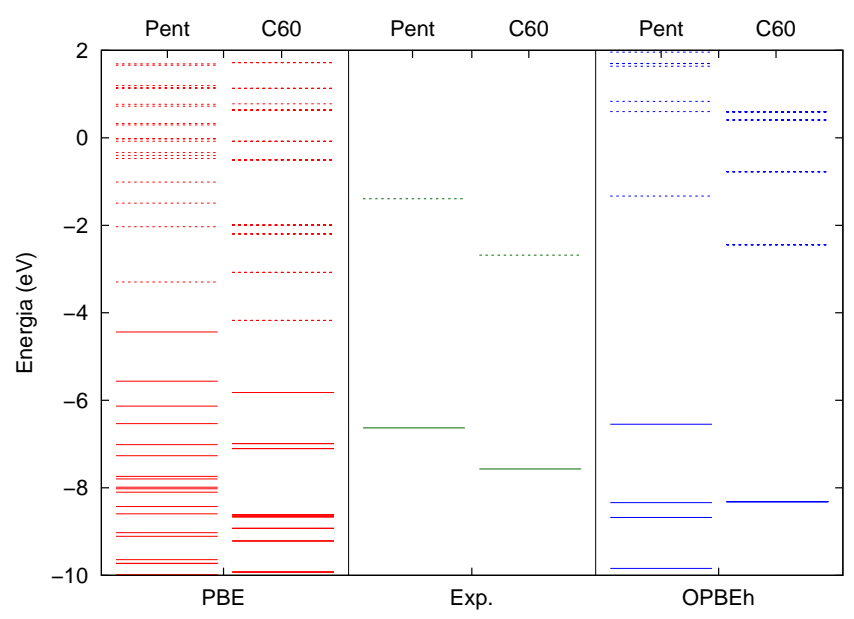

Figura 4.10: Diagrama de alinhamento dos níveis de energia calculado com diferentes métodos teóricos para as moléculas de Pentaceno e C60 no caso não-interagente, ou seja, considerando uma separação infinita. Os valores experimentais dos IPs e EAs são incluidos para comparação. As linhas tracejadas indicam os níveis de energia dos estados virtuais.

Estudos experimentais de interfaces pentaceno/C60 utilizando técnicas de espectroscopia fotoeletrônica indicam que também neste caso há formação de dipolos na interface que modificam o alinhamento dos níveis de energia [90]. Aqui, para obtermos uma primeira idéia do efeito da interação entre dois sistemas orgânicos sobre os níveis de energia, consideramos o caso mais simples de um dímero formado por uma molécula de pentaceno e uma molécula de C60. Na configuração inicial a molécula de pentaceno é então aproximada do C60 tal que o anel central do pentaceno é posicionado paralelamente a um hexágono do C60. Em seguida, a geometria do conjunto é otimizada com o funcional $\mathrm{PBE}+\mathrm{vdW}$ até que a força máxima sobre cada átomo do sistema seja inferior a $5 \mathrm{meV} / \AA$. Esses cálculos foram realizados com o conjunto de base tier 2. Devido a interação entre os sistemas, notamos que o pentaceno passa de uma geometria planar a uma estrutura levemente encurvada sobre a molécula de C60. A distância mínima obtida entre o pentaceno e o C60 é de 3,1 A.

$\mathrm{Na}$ análise a seguir, calculamos a estrutura eletrônica do dímero pentaceno/C60 em sua geometria relaxada utilizando os funcionais PBE e OPBEh. Em ambos os casos, os orbitais moleculares de fronteira HOMO e LUMO, mostrados na Fig. 4.11, confirmam o caráter doador e aceitador do pentaceno e C60, respectivamente. Os resultados obtidos com 
PBE mostram que a energia do LUMO, orbital localizado sobre o C60, aumenta de 0,20 eV devido a interação com o pentaceno. No caso do pentaceno, a variação do nível HOMO devido à mudança de geometria é desprezível, enquanto a interação intermolecular com o C60 contribui para baixar a energia do HOMO em 0,21 eV quando comparado ao valor calculado para o pentaceno isolado na mesma geometria do dímero. Isto resulta em um gap HOMO-LUMO de 0,66 eV para o dímero calculado com PBE, que é aproximadamente $0,39 \mathrm{eV}$ maior que a barreira de energia $\mathrm{HOMO}_{D}-\mathrm{LUMO}_{A}$ obtida para os sistemas isolados. Portanto, os cálculos PBE indicam que a interação intermolecular contribui para aumentar o gap de energia necessário para transferir elétrons do pentaceno para o C60.
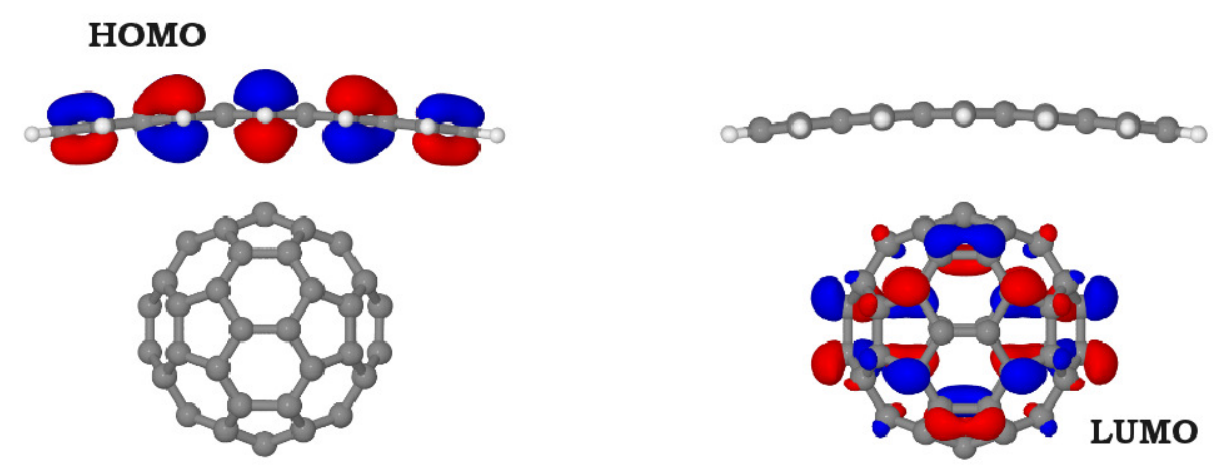

Figura 4.11: Representação esquemática dos orbitais moleculares de fronteira, HOMO e LUMO, calculados com o funcional OPBEh para o dímero Pentaceno-C60.

Tabela 4.5: Propriedades eletrônicas calculadas com diferentes funcionais para o dimero Pentaceno-C60.

\begin{tabular}{rccc}
\hline & $\mathrm{IP}(\mathrm{eV})$ & $\mathrm{EA}(\mathrm{eV})$ & $\mu_{z}(\mathrm{D})$ \\
\hline PBE & 4,64 & 3,98 & 2,18 \\
OPBEh & 6,70 & 2,26 & 1,68 \\
\hline
\end{tabular}

Qualitativamente, os cálculos OPBEh para o dímero seguem a mesma tendência dos resultados PBE, isto é, a energia do LUMO [HOMO] do sistema interagente aumenta [diminui], resultando em um aumento do gap HOMO-LUMO, quando comparado a diferença de energia $\mathrm{HOMO}_{D}-\mathrm{LUMO}_{A}$ das moléculas isoladas. O gap de energia HOMO-LUMO obtido com OPBEh é de 4,4 eV, cerca de 3,8 eV maior que o valor calculado com o funcional PBE. Notamos também que, em ambos os modelos, a interação pentaceno-C60 induz um momento 
de dipolo ao longo do eixo z (perpendicular ao plano da molécula de pentaceno) orientado do C60 para o pentaceno. De acordo com os valores listados na tabela 4.4, a inserção de troca exata no funcional OPBEh diminui o dipolo induzido no dímero. Esses resultados são consistentes com os cálculos semi-empíricos e DFT apresentados na referência 91.

Os resultados apresentados neste capítulo mostram que o funcional otimizado OPBEh, que inclui uma fração de $80 \%$ de troca HF, pode ser aplicado com êxito na determinação de potenciais de ionização e afinidades eletrônicas de semicondutores orgânicos conjugados. Embora a precisão no cálculo destas propriedades seja dependente das características estruturais dos sistemas moleculares, a concordância do nosso modelo OPBEh com o experimento é superior seja à do método HF ou à de funcionais DFT convencionais (PBE e PBE0). Ademais, mostramos para alguns casos simples, que o OPBEh pode ser utilizado também no estudo de interfaces entre diferentes sistemas orgânicos com estimativas razoavelmente boas para a barreira de transferência de carga. Entretanto, a arquitetura usual dos dispositivos orgânicos envolve a interação em interfaces entre a camada orgânica e o substrato metálico utilizado como eletrodo. Uma vez que as propriedades eletrônicas dos metais são substancialmente distintas daquelas observadas em sistemas orgânicos é de grande importância analisar a influência da troca exata sobre a estrutura eletrônica desses materiais. Esses aspectos serão discutidos no capítulo a seguir para dois metais comummente utilizados como eletrodos em dispositivos orgânicos. 



\section{Capítulo 5}

\section{Interfaces Polímero/Metal: Problemas da DFT}

A arquitetura usual dos dispositivos orgânicos é composta por um sistema em camadas no qual um filme fino de polímero conjugado é montado entre dois eletrodos, muitas vezes com interface direta polímero/metal [92,93]. A eficiência destes dispositivos depende criticamente da morfologia, composição química e estrutura eletrônica das interfaces, uma vez que as taxas de injeção de carga e outras características básicas são diretamente influenciadas pelo acoplamento eletrônico, interação química e alinhamento de bandas nas interfaces híbridas metal/polímero [94]. Do ponto de vista experimental, existe ainda dificuldade em utilizar ferramentas de análise específicas que sejam capazes de acessar as propriedades interfaciais, diferenciando-as das volumétricas. Do lado teórico, estudos que proporcionem um entendimento a nível atomístico dos processos físicos e químicos que ocorrem na interface dos dispositivos são muito importantes, e enfrentam também dificuldades específicas [95].

Diversos trabalhos tem sido publicados reportando cálculos de primeiros princípios baseados na DFT para moléculas orgânicas (finitas) adsorvidas sobre diferentes superfícies metálicas 96 101. Entretanto, a modelagem teórica da interação entre polímeros e superfícies metálicas envolve problemas distintos e não facilmente sanáveis, como veremos. De qualquer forma, na modelagem de interfaces polímero/sólido devemos primeiramente investigar 102 se existe alguma configuração favorável, normalmente uma morfologia de adsorção em que temos comensurabilidade entre as redes de um e outro sistema. Uma vez escolhido os modelos, a passagem ao estudo eletrônico pode ser extremamente difícil. 
Neste capítulo, discutimos inicialmente a adequação dos modelos DFT, focalizando os efeitos da troca exata, na descrição das propriedades eletrônicas do ouro $(\mathrm{Au})$, um metal bastante utilizado como eletrodo metálido em dispositivos orgânicos 103. Os cálculos foram realizados para Au na forma de sólido cristalino (bulk) e também na configuração de fatias (slab) para simulações de superfícies, especificamente a superfície (111). Como ficará claro no decorrer deste capítulo, fica comprovada a impossibilidade de utilizar DFTh para o metal, e assim deste ponto em diante os estudos retornam ao uso da DFT. Em seguida, passamos ao estudo da interação Polímero/Au: agora, para melhor clareza quanto aos problemas da DFT, incluímos além do polímero conjugado TPA um outro polímero também quase-1D mas não-conjugado, o polietileno (PE). Ambos mostram ter comensurabilidade com a rede da superfície $\mathrm{Au}(111)$. Para as interfaces TPA/Au e PE/Au, analisamos primeiramente a morfologia do estado fundamental via cálculos DFT incluindo correções de van der Waals (vdW). As curvas de potencial de interação polímero/Au foram obtidas para cada sistema, e a partir destas determinamos a distância de equílibrio e a energia de ligação dos polímeros adsorvidos na superfície metálica. Finalmente, considerando as geometrias de equilíbrio, analisamos a estrutura eletrônica das interfaces TPA/Au(111) e PE/ $\mathrm{Au}(111)$ focando sobre o problema da transferência de carga na DFT. Como veremos, a DFT prevê uma transferência de carga artificial para a interface TPA/Au devido à descrição incorreta dos níveis eletrônicos do polímero. Ao melhor do nosso conhecimento, este é o primeiro estudo quântico já feito para interfaces entre um polímero conjugado e um metal, no qual as condições periódicas de contorno são aplicadas a ambos os sistemas de forma que o orgânico seja representado por uma cadeia polimérica infinita.

\subsection{Propriedades Estruturais do Au - DFT}

\subsubsection{Bulk}

A descrição teórica de um sólido cristalino, bulk, é obtida aplicando-se condições periódicas de contorno a uma célula unitária representativa. No caso do Au, as estruturas de mais baixa energia possuem a simetria de uma rede de Bravais cúbica de face centrada (fcc), e portanto podem ser representadas por uma célula unitária primitiva contendo um átomo por célula, conforme esquematizado na Fig. 5.1(a). Estes sólidos metálicos possuem um volume de equilíbrio bem definido, $V_{0}$, o qual está relacionado a constante da rede periódica 
$a_{0}$. Distorções no parâmetro de rede com respeito ao valor de equilíbrio podem resultar em mudanças significativas na estrutura eletrônica do sólido. Portanto, a validação dos métodos DFT quanto ao cálculo das propriedades estruturais é de suma importância para a descrição correta da estrutura eletrônica dos metais.

A estabilidade do cristal pode ser estimada através da energia coesiva que corresponde à energia necessária para desmembrar o sólido em seus elementos constituintes. Matematicamente, a energia coesiva é expressa como a diferença na energia total do sólido cristalino e do átomo isolado:

$$
E_{\text {coe }}=\left[E_{\text {bulk }}-N E_{\text {atom }}\right] / N
$$

Diversos estudos teóricos foram publicados em anos recentes comparando a eficiência de diferentes modelos DFT na descrição das propriedades estruturais e coesivas de sólidos metálicos [30,104, 105, a seguir apresentamos os resultados DFT obtidos com o programa FHI-aims para as propriedades estruturais e coesivas do cristal de $\mathrm{Au}$, com os diferentes os funcionais de troca e correlação LDA-PW, PBE e PBE+vdW. Simulamos o bulk do metal como um sólido cristalino perfeito construído a partir da célula prmitiva fcc (ver Fig. 5.1(a)) com dimensões $\alpha=\beta=\gamma=60,0^{\circ}$ obtidas de dados experimentais [106]. Todos os cálculos foram realizados com o conjunto de base numérico tight/tier 1 .

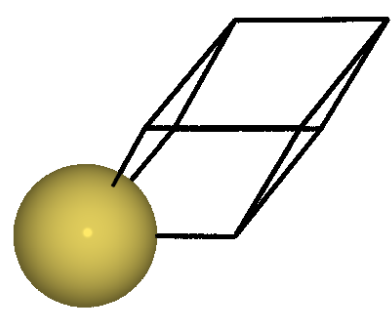

(a) primitiva

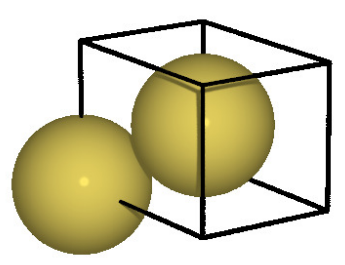

(b) ortorrômbica

Figura 5.1: Modelos de célula unitária para um sólido cristalino de ouro com simetria cúbica de face centrada (FCC).

Em metais, o número de ocupação das bandas de energia que cruzam o nível de Fermi é descontínuo como função do vetor de onda k. Consequentemente, a ocupação dos autoestados pode oscilar durante as iterações de campo auto-consistente, resultando em uma convergência muito lenta das integrais com o número de pontos k. O procedimento matemático utilizado para resolver este problema é a técnica de smearing na qual os níveis 
eletrônicos são alargados por uma função suave (Fermi, Methfessel-Paxton ou gaussiana) permitindo ocupações parciais dos estados próximos ao nível de Fermi. Nos cálculos apresentados a seguir, as integrações na primeira zona de Brillouin são realizadas em uma malha de 21x21x21 pontos k com um esquema de ocupação gaussiano (smearing $0,1 \mathrm{eV}$ ).

Devido à grande carga nuclear do ouro, os efeitos relativísticos tornam-se importantes e são tratados em nossos cálculos através do método zeroth-order regular approximation (ZORA) [107], no qual o Hamiltoniano ZORA é obtido como uma equação de ordem zero na expansão regular em E/ $\left(2 \mathrm{c}^{2}-\mathrm{v}\right)$ da equação de Dirac. Especificamente no caso da aproximação scaled ZORA, utilizada nesta Tese, as energias de uma partícula são re-escaladas após a auto-consistência utilizando a expressão

$$
\epsilon_{l}^{\text {scaled }}=\frac{\epsilon_{l}^{Z O R A}}{1+\left\langle\psi_{l}\left|\mathbf{p} \frac{c^{2}}{\left(2 c^{2}-v\right)^{2}} \mathbf{p}\right| \psi_{l}\right\rangle}
$$

onde $\mathbf{p}=-i \nabla$ é o operador momento. A energia total do sistema é escrita como uma soma dos autovalores ZORA escalados $\epsilon_{l}^{\text {scaled }}$. A descrição detalhada do método ZORA implementado no FHI-aims é encontrada na referência [51.

Primeiramente, determinamos o parâmetro $a_{0}$ de equilíbrio para cada funcional por meio de um cálculo de relaxação estrutural, minimizando a energia total até que a força máxima com relação ao átomo e aos vetores da rede seja inferior a $5 \mathrm{meV} / \AA$. Neste cálculo fixamos os ângulos $\alpha=\beta=\gamma$ da célula primitiva de forma a preservar a simetria fcc da rede. Em seguida, calculamos a energia de coesão dos metais conforme a equação 5.1. Para determinar a energia total do átomo livre realizamos cálculos SCF spin-polarizado com os mesmos parâmetros computacionais utilizados no cálculo do sólido, isto é, mesmo funcional e conjunto de base.

As constantes de rede e energias coesivas do Au cristalino obtidas com diferentes funcionais são listadas na tabela 5.1 incluindo dados experimentais para comparação. Entretanto, ressaltamos que os dados experimentais mostrados na tabela foram obtidos a temperatura ambiente $(\mathrm{T}=300 \mathrm{~K})$ e portanto incluem efeitos de expansão térmica não tratados em nossos cálculos. Os resulados da tabela mostram que, em geral, os modelos DFT padrão descrevem razoavelmente bem a estrutura do metal com erro inferior a $2 \%$ para o cálculo da constante de rede $a_{0}$. Comparado à constante de rede experimenal, o método LDA tende a subestimar o parâmetro $a_{0}$ como esperado, pois este modelo superestima as forças de ligação do 
Tabela 5.1: Constantes de rede de equilíbrio $(\AA)$ e energias de coesão (eV) do sólido cristalino $\mathrm{Au}$, calculadas utilizando o programa FHI-aims com diferentes funcionais. Os valores são comparados com dados experimentais obtidos à temperatura ambiente.

\begin{tabular}{lcc}
\hline & $a_{0}$ & $E_{\text {coe }}$ \\
\hline LDA & 4,05 & $-4,36$ \\
PBE & 4,15 & $-3,05$ \\
PBE + vdW & 4,11 & - \\
\hline Exp. 106 & 4,078 & $-3,81$ \\
\hline
\end{tabular}

material, e assim a LDA prevê estruturas mais fortemente ligadas, isto é, com energias de coesão mais baixas. Entretanto, notamos também que, entre os funcionais testados, a LDA resulta na melhor descrição para a estrutura do $\mathrm{Au}$, com erros menores tanto no cálculo da constante de rede quanto da energia coesiva. Em contraste à LDA, os métodos GGA tendem a superestimar a constante de rede de sólidos metálicos, conforme demonstrado em estudos teóricos anteriores 104, 108, e os resultados apresentados na tabela 5.1 confirmam esse comportamento.

Cálculos teóricos para a constante de rede e energia coesiva de metais bulk tem sido publicados utilizando diferentes métodos e níveis de aproximação. No caso do Au, por exemplo, Schimka et al. [109 calcularam com o método PAW-PBE a constante de rede e energia coesiva do Au bulk obtendo os valores 4,154 $\AA$ e 3,05 eV. Nossos resultados são portanto consistentes com outros cálculos da literatura teórica.

Em sólidos não-covalentes tal como no caso do $\mathrm{Au}$, as forças de dispersão ausentes nos métodos DFT usuais são especialmente importantes para a descrição da estrutura e coesão desses sistemas [105. Adicionando-se a correção de van der Waals ao funcional PBE, notamos que a constante de equílibrio $a_{0}$ do Au bulk é reduzida em 0,04 $\AA$, melhorando sensivelmente o acordo com o dado experimental.

Os resultados discutidos acima mostram que os funcionais DFT padrão fornecem uma descrição razoável para a estrutura de sólidos metálicos. Em particular, a geometria PBE dos cristais apresenta apenas pequenos desvios em relação ao experimento. Assim, a constante de rede DFT pode ser utilizada como uma boa aproximação para representar a estrutura de equilíbrio dos metais. Além disso, conforme será mostrado adiante, o parâmetro de rede experimental dos polímeros estudados neste trabalho é comensurável com os das superfícies 
metálicas, permitindo que as condições periódicas sejam aplicáveis a ambos os sistemas. Devido a este fato, e também para manter a coerência com a escolha do parâmetro de rede experimental feita para o TPA, consideraremos apenas a geometria experimenal dos metais como padrão para o estudo da estrutura eletrônica e construção dos modelos de superfícies nos cálculos apresentados a seguir.

\subsubsection{Superfície}

Em geral, a estrutura das superfícies metálicas apresentam características específicas visto que qualquer átomo localizado na superfície do metal encontra um ambiente espacial diferente do átomo no bulk. Efeitos de relaxação vertical e reconstrução horizontal podem portanto ocorrer em uma superfície mesmo partindo da monocristalina ideal. Em particular, as características estruturais e eletrônicas das superfícies metálicas dependem da orientação cristalográfica e dimensões do sistema. Nesta seção apresentamos um estudo DFT das propriedades estruturais de fatias (slabs) de Au, modelados como superfícies ideais, ou seja, sem considerar possíveis reconstruções.

No modelo de slab constrói-se uma supercélula com um número escolhido (pequeno) de camadas atômicas empacotadas em estrutura cristalina, na direção escolhida, e separadas por uma região de vácuo que impede a interação entre as fatias. A Fig. 5.2 ilustra um exemplo de supercélula utilizado neste trabalho para o sistema $\mathrm{Au}(111)$ com $\mathrm{N}=5$ camadas atômicas.

Primeiramente, construimos uma sequência de slabs, com o número de camadas atômicas variando entre $\mathrm{N}=2$ e $\mathrm{N}=12$, a partir da célula unitária ortorrômbica com corte na direção (111). Isto corresponde a uma espessura máxima de 26,09 $\AA$ para $\mathrm{Au}(111)$. A geometria dos slabs é representada em uma supercélula ortorômbica 2x2x1, cujos parâmetros de rede (determinados a partir do cristal experimental) paralelo aos planos atômicos são a=5,77 $\AA$ e b=4,997 $\AA$. Para minimizar a interação entre as imagens periódicas dos slabs, a constante de rede perpendicular a superfície foi fixada em $\mathrm{c}=80,0 \AA$ que corresponde a uma região de vácuo de no mínimo $54 \AA$.

A estrutura de equilíbrio de cada slab foi obtida através de cálculos de otimização de geometria no qual a energia total do sistema é minimizada com respeito à todas as coordenadas atômicas, exceto à coordenada $\mathrm{z}$ dos átomos pertencentes à primeira camada (camada inferior que não entrará em contato com o orgânico), fixada em $z=0$ durante toda a simu- 


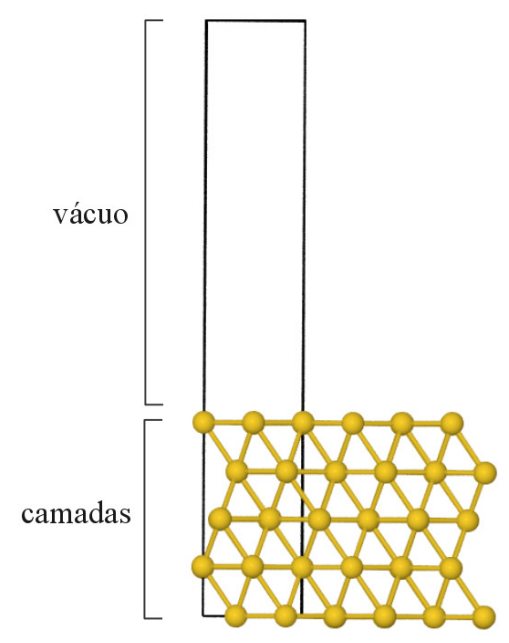

(a) lateral

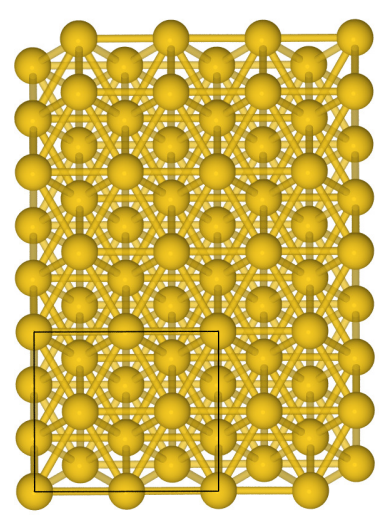

(b) superior

Figura 5.2: Representação esquemática de um slab referente à superficie (111) do cristal de $\mathrm{Au}$, nas visões lateral e superior. A célula unitária utilizada é destacada com linhas preenchidas.

lação. Estes cálculos foram realizados com o funcional $\mathrm{PBE}+\mathrm{vdW}$ utilizando o conjunto de base numérico tier 1 . Os demais parâmetros computacionais foram mantidos iguais aos previamente descritos para o cálculo do bulk.

O espaçamento entre duas camadas adjacentes para slabs não-relaxados de um cristal fcc é dado por $a_{0} / \sqrt{3}$ que, no caso do Au, resulta em uma separação de 2,354 A. Após o cálculo de otimização de geometria, para o maior slab considerado nesse estudo $(\mathrm{N}=12)$, a separação média entre camadas obtida foi de 2,372 A. Isto corresponde a uma expansão de 0,22 Å na espessura do slab de Au.

Na tabela 5.2 listamos os valores calculados da variação no espaçamento entre camadas como resultado da relaxação da superfície $\mathrm{Au}(111)$ para slabs contendo $\mathrm{N}=5,6,9$ e 12 camadas, respectivamente. A relaxação intercamadas, $\Delta d_{i, i+1}$, é dada em termos percentuais pela expressão,

$$
\Delta d_{i, i+1}=100\left(d_{i, i+1}-d_{0}\right) / d_{0}
$$

onde $d_{0}$ corresponde a distância entre camadas para o sistema não-relaxado, e $d_{i, i+1}$ corresponde a distância entre duas camadas adjacentes paralelas à superfície obtida após a otimização de geometria com o funcional PBE +vdW. Portanto, um sinal negativo na eq. 5.3 
Tabela 5.2: Relaxações intercamadas dadas em porcentagem, $\Delta d_{i, i+1}$, para slabs de $\mathrm{Au}(111)$ otimizadas com o funcional PBE + vdW.

\begin{tabular}{lllll}
\hline & $\mathrm{N}=12$ & $\mathrm{~N}=9$ & $\mathrm{~N}=6$ & $\mathrm{~N}=5$ \\
\hline$\Delta d_{1,2}$ & $+0,964$ & $+4,313$ & $+4,413$ & $+4,884$ \\
$\Delta d_{2,3}$ & $-0,745$ & $+1,835$ & $+1,666$ & $+2,225$ \\
$\Delta d_{3,4}$ & $-0,455$ & $+1,782$ & $+1,531$ & $+2,045$ \\
$\Delta d_{4,5}$ & $-0,089$ & $+1,877$ & $+1,737$ & $+4,316$ \\
$\Delta d_{5,6}$ & $+0,158$ & $+1,884$ & $+4,592$ & \\
$\Delta d_{6,7}$ & $+0,450$ & $+1,774$ & & \\
$\Delta d_{7,8}$ & $+0,677$ & $+1,840$ & & \\
$\Delta d_{8,9}$ & $+0,858$ & $+4,252$ & & \\
$\Delta d_{9,10}$ & $+1,016$ & & & \\
$\Delta d_{10,11}$ & $+1,326$ & & & \\
$\Delta d_{11,12}$ & $+4,189$ & & & \\
\hline
\end{tabular}

indica uma contração do espaçamento entre camadas enquanto o sinal positivo indica uma expansão.

Qualitativamente, notamos que o espaçamento entre as duas primeiras camadas apresenta um aumento independente da espessura do slab. Notamos que, para os slabs com $\mathrm{N}=5, \mathrm{~N}=6$ e $\mathrm{N}=9$, todos os espaçamentos entre planos atômicos sofrem uma expansão de forma praticamente simétrica em relação ao plano mediano, e que o maior percentual de expansão ocorre sempre para as duas últimas camadas no topo da superfície. O caso do slab com $\mathrm{N}=12$ é especial, e pode ser derivado do vínculo imposto à coordenada $\mathrm{z}$ da camada inferior, porém a expansão para as camadas superiores se confirma. A relaxação vertical intercamadas é também sensível ao modelo DFT adotado [110,111, entretanto os efeitos sobre as propriedades eletrônicas do metal pode ser desprezado [111. Utilizaremos aqui as geometrias $\mathrm{PBE}+\mathrm{vdW}$ otimizadas dos slabs metálicos como base para o estudo da estrutura eletrônica. 


\subsection{Propriedades eletrônicas - DFT versus DFTh}

\subsubsection{Bulk}

Nesta seção, apresentamos resultados para a estrutura de bandas e a densidade de estados dos metais bulk comparando os modelos DFT e DFT híbrido (DFTh), no intuito de racionalizar o efeito da troca exata sobre a estrutura eletrônica do sistema de interesse. Os resultados teóricos são também comparados a medidas experimentais disponíveis, o que permite analisar a adequação do modelo para descrever as propriedades eletrônicas do ouro.

As propriedades eletrônicas do Au cristalino foram obtidas a partir de cálculos de energia total auto-consistentes realizados com os funcionais de troca e correlação GGA/PBE e o híbrido PBE0 utilizando o conjunto de base tier 1. Para os cálculos DFT puro, a malha de pontos $\mathrm{k}$ foi aumentada para $25 \times 25 \times 25$, garantindo assim uma boa convergência da densidade eletrônica. Já no caso dos funcionais com troca exata, o elevado custo computacional dos cálculos all-electron para o Au limita a malha de pontos k em um máximo de 14x14x14, que foram então utilizados nesses cálculos. Todos os demais parâmetros computacionais foram mantidos como nos cálculos de otimização estrutural discutidos na seção 5.1.

Iniciamos nosso estudo apresentando os resultados obtidos para o cristal de ouro fcc. A Fig. 5.3 mostra as bandas de condução de energia mais baixa calculadas para a célula primitiva experimental do Au bulk na primeira zona de Brillouin ao longo das linhas de alta simetria. Qualitativamente, os cálculos DFT e DFTh reproduzem as características básicas da estrutura de bandas dos metais $d$ : formação de cinco bandas ocupadas com pequena dispersão provenientes dos estados $5 d$, e uma banda de caráter $s$ semi-preenchida com grande dispersão, que cruza o nível de Fermi em diferentes pontos.

O principal efeito da troca exata sobre a estrutura de bandas do Au é deslocar para baixo os níveis ocupados $5 d$ de forma quase-rígida, enquanto a primeira banda totalmente desocupada é deslocada para cima de 1,6 eV. Isto resulta em um aumento dos gaps internos $5 d-6 s p$. Este efeito é detalhado na tabela 5.3 , onde incluímos para comparação resultados $\mathrm{G}_{0} \mathrm{~W}_{0} @ \mathrm{PBE}$ da literatura 112 . Dado o deslocamento quase-rígido das bandas $d$, as mudanças nos gaps se tornam mais significativas para transições envolvendo bandas com caráter predominantemente $s p$ como, por exemplo, no caso do gap $\mathrm{L}_{3^{\prime}} \rightarrow \mathrm{L}_{2^{\prime}}$, ou do $\mathrm{X}_{5} \rightarrow \mathrm{X}_{4^{\prime}}$ que aumenta em 1,6 eV quando a fração de troca exata é inserida no funcional PBE. 

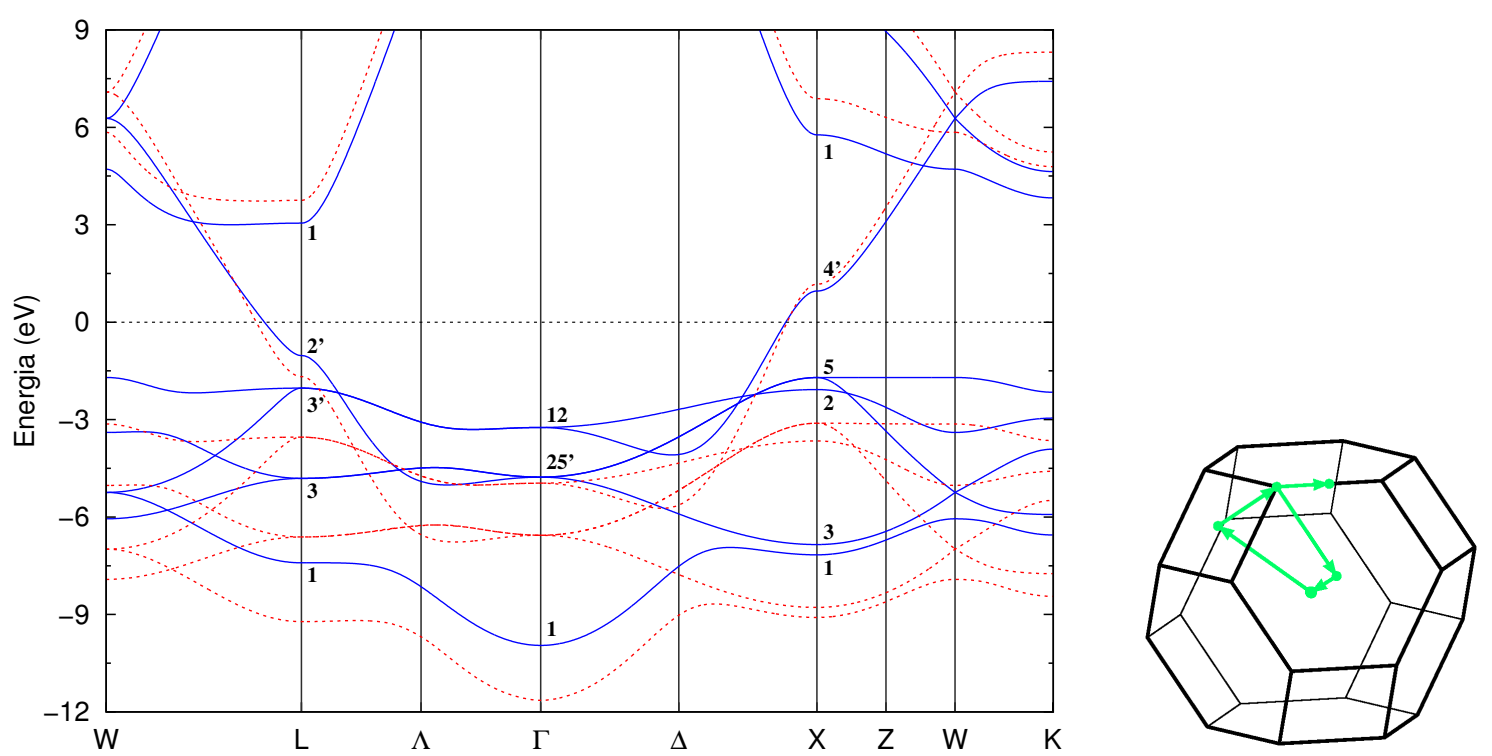

Figura 5.3: Estrutura de bandas do ouro bulk (fcc) calculada com os funcionais PBE (linha sólida azul) e PBE0 (linha vermelha pontilhada). A zona de Brillouin correspondente é também mostrada.

Cálculos de densidade de estados eletrônicos, convoluídos com gaussianas de largura $0,25 \mathrm{eV}$, foram também realizados para o Au bulk e os resultado obtidos são mostrados na Fig. 5.4juntamente com o espectro retirado da referência experimental 113. Primeiramente, notamos que o funcional PBE fornece a melhor descrição do perfil do espectro de fotoemissão experimental [113 quando comparado ao PBE0. Além disso, verificamos que a largura de banda calculada com PBE0 (11,0 eV) é maior que o valor PBE $(9,9 \mathrm{eV})$ e o experimento $(9,6$ $\mathrm{eV})$. A fronteira superior da banda $5 d$ é deslocado para energias mais baixas no PBE0 ( 2,4 eV abaixo do nível de Fermi) comparado ao PBE ( 1,3 eV abaixo do nível de Fermi), ficando portanto $0,7 \mathrm{eV}$ abaixo da referência experimental. Além disso, notamos os picos entre $1 \mathrm{e}$ $3 \mathrm{eV}$ a partir do nível de Fermi no espectro PBE0, que representam uma diferença marcante em relação ao espectro experimental.

As mudanças na estrutura de bandas e DOS discutidas acima estão associadas a variações na densidade eletrônica do metal devido a inclusão de troca exata no funcional PBE. Assim, analisamos estas variações calculando a diferença na densidade eletrônica total, $\Delta \rho=\rho_{P B E 0}-\rho_{P B E}$, representada graficamente pelos mapas de contorno 2D na Fig. 5.5. Vemos então que a troca exata contribui para aumentar a concentração de elétrons (região 
Tabela 5.3: Gaps de energia internos cálculados a partir da estrutura de bandas do ouro bulk ao longo das linhas de alta simetria. Os valores teóricos da referência [112 obtidos com o método GW são incluídos para comparação.

\begin{tabular}{lccccc}
\hline & LDA & PBE & PBE0 & $\mathrm{G}_{0} \mathrm{~W}_{0}$ & QSGW \\
\hline $\mathrm{L}_{3} \rightarrow \mathrm{L}_{3^{\prime}}$ & 2,82 & 2,78 & 3,07 & 2,90 & 2,80 \\
$\mathrm{~L}_{3^{\prime}} \rightarrow \mathrm{L}_{2^{\prime}}$ & 0,91 & 0,98 & 1,86 & 0,40 & 1,20 \\
$\mathrm{~L}_{2^{\prime}} \rightarrow \mathrm{L}_{1}$ & 4,06 & 4,10 & 5,43 & 4,80 & 4,60 \\
$\Gamma_{1} \rightarrow \Gamma_{25^{\prime}}$ & 5,31 & 5,18 & 5,06 & 5,60 & 5,00 \\
$\Gamma_{25^{\prime}} \rightarrow \Gamma_{12}$ & 1,55 & 1,51 & 1,64 & 1,50 & 1,50 \\
$\mathrm{X}_{3} \rightarrow \mathrm{X}_{2}$ & 4,79 & 4,75 & 5,12 & 4,80 & 4,70 \\
$\mathrm{X}_{5} \rightarrow \mathrm{X}_{4^{\prime}}$ & 2,57 & 2,65 & 4,27 & 2,30 & 3,10 \\
$\mathrm{X}_{4^{\prime}} \rightarrow \mathrm{X}_{1}$ & 4,76 & 4,79 & 5,72 & 5,40 & 5,20 \\
\hline
\end{tabular}

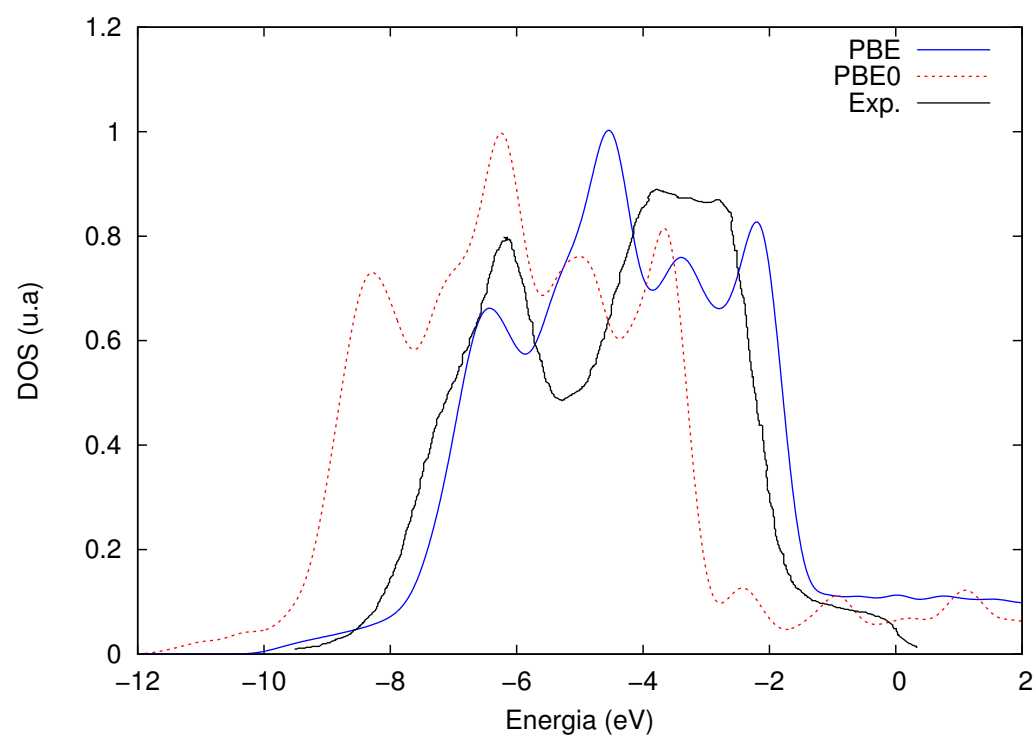

Figura 5.4: Densidade de estados eletrônica do ouro bulk (fcc) na região de condução calculada com os funcionais PBE e PBE0 utilizando o conjunto de base tier 1. O espectro de fotoemissão experimental (XPS) é incluído para comparação.

vermelha) nas regiões intersticiais dos íons da rede. A distribuição dos elétrons de camadas mais profundas é também afetada de forma não-homogênea pela troca exata, conforme indicado pelo pequeno círculo vermelho na região próxima ao núcleo. 


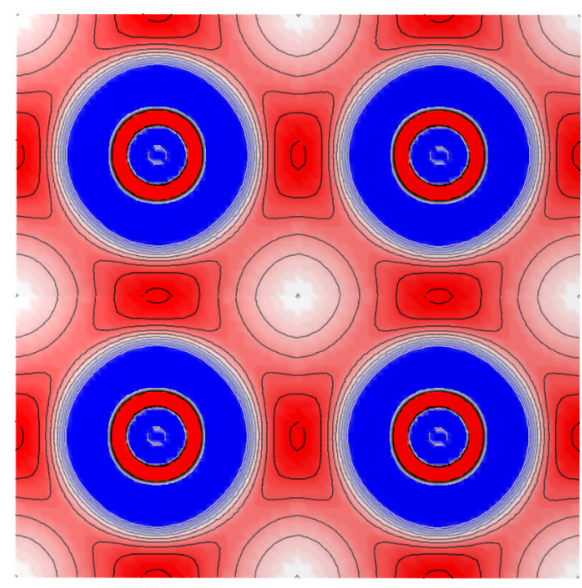

(a) plano (100)

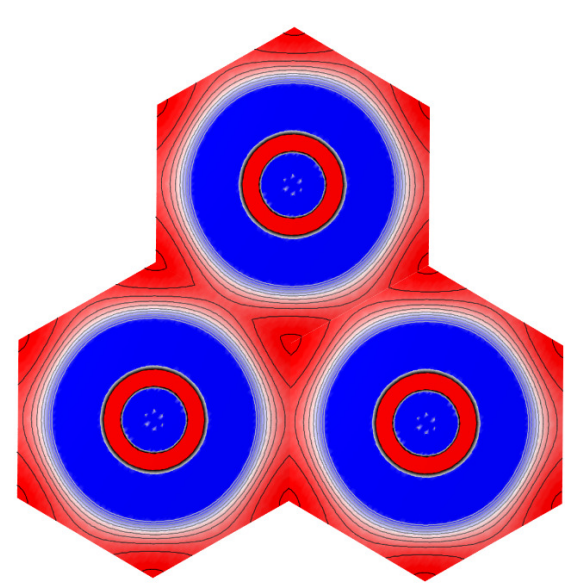

(b) plano (111)

Figura 5.5: Mapas de contorno 2D da diferença na densidade de cargas, , $\Delta \rho$, calculada com os funcionais PBE0 e PBE. Os valores mínimo e máximo $\left( \pm 0,006 \mathrm{e}^{-3}\right)$ das isosuperfícies são representados nas cores vermelho e azul, e indicam as regiões de acumulo e perda de elétrons, respectivamente.

Embora os funcionais híbridos melhorem a descrição das propriedades estruturais de sólidos em geral 114,115 comparado aos métodos DFT convencionais, vimos que estes funcionais são inadequados para uma descrição precisa da estrutura eletrônica de sistemas metálicos. Nesses sistemas, a blindagem dielétrica é mais intensa e portanto a fração ótima de troca exata necessária para descrever corretamente a estrutura eletrônica deve ser, em princípio, menor que os $25 \%$ utilizado no funcional PBE0. Vimos também que o efeito global da troca exata é deslocar os níveis eletrônicos ocupados para energias mais baixas, deslocando as bandas $d$ e alargando a banda $s$, e aumentar os gaps. Especificamente no caso do $\mathrm{Au}$, nossos cálculos mostraram que também a energia de Fermi é sensível à troca exata, variando em até $0,5 \mathrm{eV}$ quando passamos do funcional PBE para o PBE0.

\subsubsection{Superfície}

A simulação de superfícies metálicas utilizando um modelo de fatias com diferentes espessuras leva a um comportamento oscilatório para diferentes propriedades físicas do sistema devido a efeitos de confinamento quântico 116 . Em particular, tem sido observado em estudos teóricos anteriores 110,111,117] que a função trabalho de superfícies metálicas monocristalinas pode oscilar como função da espessura das camadas. Uma vez que estamos 
interessados no estudo de superfícies é importante determinar a espessura mínima dos slabs para o qual as propriedades físicas de interesse estejam suficientemente convergidas.

A função trabalho, $\phi$, é a energia mínima necessária para remover um elétron a partir do bulk de um material através da superfície para um ponto no vácuo imediatamente fora da superfície sólida. Em geral, esta propriedade depende da simetria e empacotamento específicos da superfície cristalina. Na prática, $\phi$ corresponde a energia necessária à $0 \mathrm{~K}$ para remover um elétron do nível de Fermi do metal para o potencial de vácuo. Cálculos de função trabalho utilizando DFT seguem esta definição, tal que o valor de $\phi$ é dado pela diferença entre o potencial eletrostático em um ponto distante da superfície e a energia de Fermi, $\phi=V_{e s}(\infty)-E_{F}$, a partir de cálculos realizados para slabs de metal em uma geometria específica.

As estruturas de equilíbrio dos slabs de $\mathrm{Au}(111)$ previamente otimizadas com o funcional $\mathrm{PBE}+\mathrm{vdW}$ foram utilizadas como geometrias iniciais para os cálculos de energia total SCF utilizando diferentes funcionais. Neste caso, o conjunto de base foi aumentado para tier 2 para obtermos uma descrição mais precisa das propriedades eletrônicas.

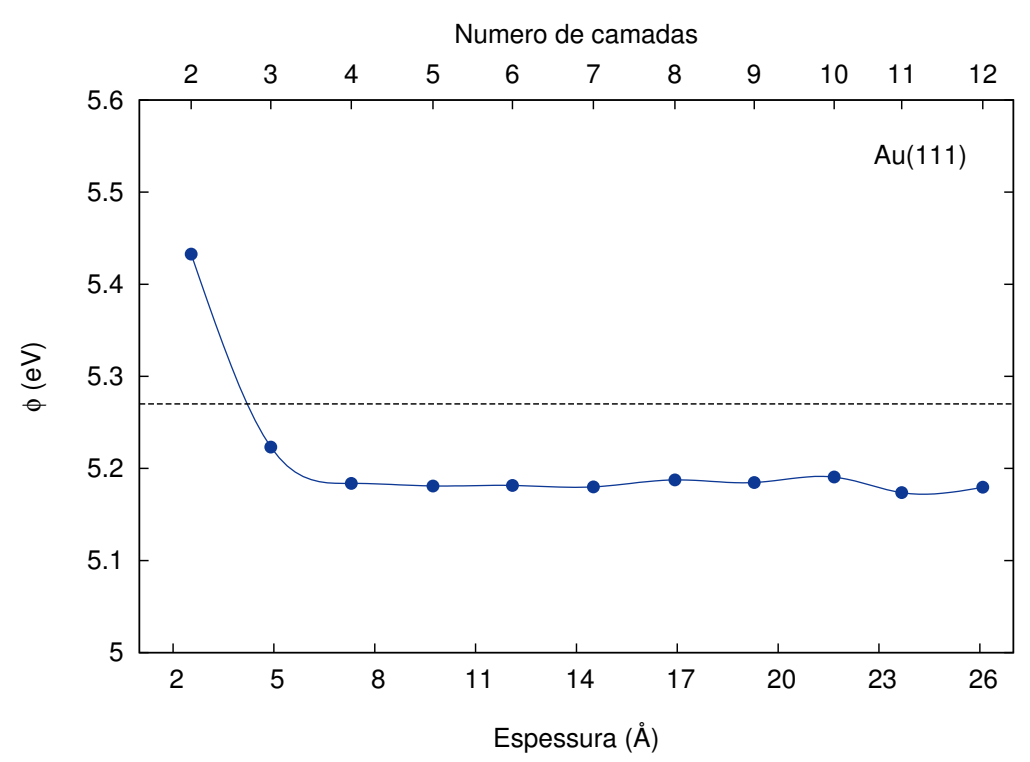

Figura 5.6: Função trabalho das superfícies relaxadas de $\mathrm{Au}(111)$ como função da espessura das camadas. Os cálculos foram realizados com o funcional DFT-PBE na base tier 2. As linhas tracejadas indicam o valor de referência experimental. 
A função trabalho dos slabs de $\mathrm{Au}(111)$ calculada como função do número de camadas no slab é apresentada na Fig. 5.6. Notamos que a função trabalho do Au converge rapidamente apresentando apenas pequenas flutuações em torno do valor médio de $5,18 \mathrm{eV}$ para $\mathrm{N}>3$ camadas. Um comportamento similar foi observado nos cálculos DFT de Singh-Miller e Marzari 110 para a superfície de $\operatorname{Pd}(100)$. Neste mesmo trabalho, os autores obtiveram um valor de $\phi=5,15 \mathrm{eV}$ para a função trabalho da superfície $\mathrm{Au}(111)$ que está em bom acordo com nossos resulados. Além disso, o valor convergido PBE da função trabalho é apenas 0,08 eV menor que o valor experimental obtido por fotoemissão [118.

\subsection{Interfaces Polímero/Au: TPA e PE}

O processo de adsorção em interfaces orgânico-metal, fracamente interagentes, é dominado por forças de dispersão [119] que não são precisamente descritas por funcionais DFT convencionais. A razão disto é que a interação vdW é não-local e de longo alcance, enquanto os funcionais de troca e correlação na aproximação LDA ou GGA são locais e de curto alcance. Assim, para a descrição da morfologia das interfaces OM dentro do formalismo DFT é necessário o uso de modelos que incluam adequadamente as interações de van der Waals 120,121. À parte essa necessidade, notamos também que em interfaces híbridas orgânico-metal (OM), os detalhes da estrutura eletrônica dependem criticamente da geometria específica que o polímero ou molécula assumem ao interagir com o substrato. Em particular, o acoplamento eletrônico entre o componente orgânico e o substrato metálico é bastante sensível à separação vertical entre os dois sub-sistemas e vice-versa [122], ou seja, se houver transferência de carga de um para outro material pode haver também um efeito na morfologia.

A tabela 5.4 apresenta valores de potencial de ionização e afinidade eletrônica de oligômeros finitos calculados com diferentes funcionais que já ilustram a seriedade do problema no tratamento de polímeros conjugados infinitos sobre Au (ou outro metal) através da DFT. Tomamos como exemplo os acetilenos já discutidos no capítulo 2, e incluímos também o decano, oligo-etileno com 10 átomos de carbono. Enfatizamos novamente que para o OTA5 o funcional PBE leva a um IP mais baixo que o (realista $\mathrm{G}_{0} \mathrm{~W}_{0} @ \mathrm{OPBEh}$ ) por 2,5 eV. Nossa preocupação vem da comparação entre o IP do OTA5 e a função $\phi$ do metal, pois teremos um desajustamento grave dos níveis de fronteira com os valores PBE: enquanto esperaríamos que o "topo da valência"(HOMO) do orgânico estivesse abaixo da energia de Fermi do 
Tabela 5.4: Valores teóricos para a energia de ionização e afinidade eletrônica de moléculas orgânicas calculadas com diferentes formalismos teóricos.

\begin{tabular}{lccccc}
\hline \multicolumn{5}{c}{ Potencial de Ionização $(\mathrm{eV})$} \\
\hline fórmula & PBE & PBE0 & OPBEh & $\mathrm{G}_{0} \mathrm{~W}_{0} @$ OPBEh \\
\hline OTA3 & $\mathrm{C}_{6} \mathrm{H}_{8}$ & 5,31 & 6,13 & 8,09 & 8,17 \\
OTA5 & $\mathrm{C}_{10} \mathrm{H}_{12}$ & 4,87 & 5,57 & 7,34 & 7,36 \\
Decano & $\mathrm{C}_{10} \mathrm{H}_{22}$ & 7,08 & 8,38 & 11,34 & 10,82 \\
\hline \multicolumn{5}{c}{ Afinidade Eletrônica $(\mathrm{eV})$} \\
\hline OTA3 & $\mathrm{C}_{6} \mathrm{H}_{8}$ & 2,47 & 1,64 & $-0,27$ & $-0,52$ \\
OTA5 & $\mathrm{C}_{10} \mathrm{H}_{12}$ & 2,88 & 2,18 & 0,45 & 0,28 \\
Decano & $\mathrm{C}_{10} \mathrm{H}_{22}$ & 0,23 & $-0,40$ & $-1,62$ & $-1,64$ \\
\hline
\end{tabular}

metal, ocorrerá o contrário o que promoveria transferência eletrônica diretamente do HOMO do OTA5 para os estados disponíveis no metal, vinda artificialmente do método de cálculo. Esse problema pode ser ainda agravado para sistemas com baixo valor de gap como é o caso do TPA.

Notamos que muitos trabalhos recentes têm sido realizados e publicados tratando moléculas (finitas) conjugadas sobre metais, em particular ouro 123 125. O que vemos na tabela traz também o motivo pelo qual, muitas vezes, os problemas assinalados acima não ocorrem: por exemplo para o $\pi$-conjugado OTA3 (mesmo número de carbonos que o benzeno) o valor do IP obtido com o PBE está ainda na "faixa de segurança"para impedir a transferência espúria, ou artificial, de carga do orgânico ao metal.

O efeito de hibridização ( $\pi$-conjugado vs $s p^{3}$ ) vem também claramente assinada na diferença de gap e IP entre o OTA5 e o decano (mesmo número de carbonos): o problema de transferência artificial de cargas também não ocorre para o decano, já que o IP obtido com qualquer funcional é mais fundo que a energia de Fermi do Au.

A seguir faremos o estudo das ínterfaces polímero/metal, mas tendo já em mente os problemas inerentes ao método PBE.

\subsubsection{Propriedades Estruturais - DFT}

Em consonância com o objetivo de distinção entre características eletrônicas das interfaces com orgânicos conjugados e saturados, estudamos aqui além do TPA também o polietileno $(\mathrm{PE})$, formado por uma cadeia linear de carbonos saturados do tipo $s p^{3}$. As diferenças na 
estrutura química ditam as propriedades óticas e eletrônicas dos polímeros tal que o TPA se comporta como um semicondutor com baixo gap de energia, e o PE é tipicamente um material isolante.

Para ambos os materiais, TPA e PE, temos comensurabilidade entre as redes periódicas do polímero e a do metal Au. Isto significa que a constante de rede do polímero, que é caracterizado pelo tamanho do monômero, é aproximadamente um múltiplo inteiro de um dos parâmetros de rede da superfície metálica $\mathrm{Au}(111)$. Por exemplo, a célula ortorômbica mínima da superfície $\mathrm{Au}(111)$ experimental possui uma constante de rede de $\mathrm{b}=4,997 \AA$ que é cerca de duas vezes o comprimento de um monômero, $\mathrm{c}_{T P A}=2,457 \AA$ e $\mathrm{c}_{P E}=2,534 \AA$. De fato, vemos que a diferença entre a constante de rede $b$ da superfície $\mathrm{Au}(111)$ experimental e o comprimento de um dímero (2c) é inferior a 1,8\%. Consequentemente, o polímero pode ser reproduzido com condições periódicas sobre a mesma rede do metal sem grandes distorções em sua geometria e propriedades eletrônicas.

Modelamos a interface polímero/metal (P/M) utilizando como substrato a superfície $\mathrm{Au}(111)$ construída a partir da célula ortorômbica experimental do bulk. A superfície metálica é representada pelo slab de 5 camadas atômicas para o qual a morfologia das camadas mais externas, e também o valor da função trabalho já são bem representadas, como visto anteriormente. As cadeias de TPA e PE são posicionadas a uma altura z da superfície metálica com o plano dos carbonos paralelo à superfície e alinhados ao longo do parâmetro $b$ do metal. Sendo assim, o sistema polímero/Au(111) contém uma cadeia por célula periódica formando então uma monocamada de polímero (TPA e PE) adsorvida sobre a superfície metálica, conforme ilustrado na Fig. 5.7.

Primeiramente, a geometria de equílibrio de cada interface $\mathrm{P} / \mathrm{M}$ foi obtida por meios de cálculos de otimização DFT+vdW para uma célula periódica fixa, minimizando as forças sobre todos os átomos do polímero e do slab metálico até um valor inferior a $0,005 \mathrm{eV} / \AA$. Estes cálculos foram realizados com o funcional de troca e correlação PBE adicionando correções de disperção baseadas no modelo TS modificado para incluir também efeitos de blindagem dos elétrons do substrato na determinação dos parâmetros de van der Waals [126], $\mathrm{PBE}+$ scvdW. De acordo com este método, o coeficiente da interação vdW que envolve um átomo do sistema orgânico e um átomo do metal depende da função dielétrica do metal.

Para representar as funções de onda, utilizamos o conjunto de base padrão tight que inclui a base tier 2 para elementos leves $(\mathrm{C}$ e $\mathrm{H})$ e tier 1 para o Au. Os efeitos relativísticos foram 
(a)
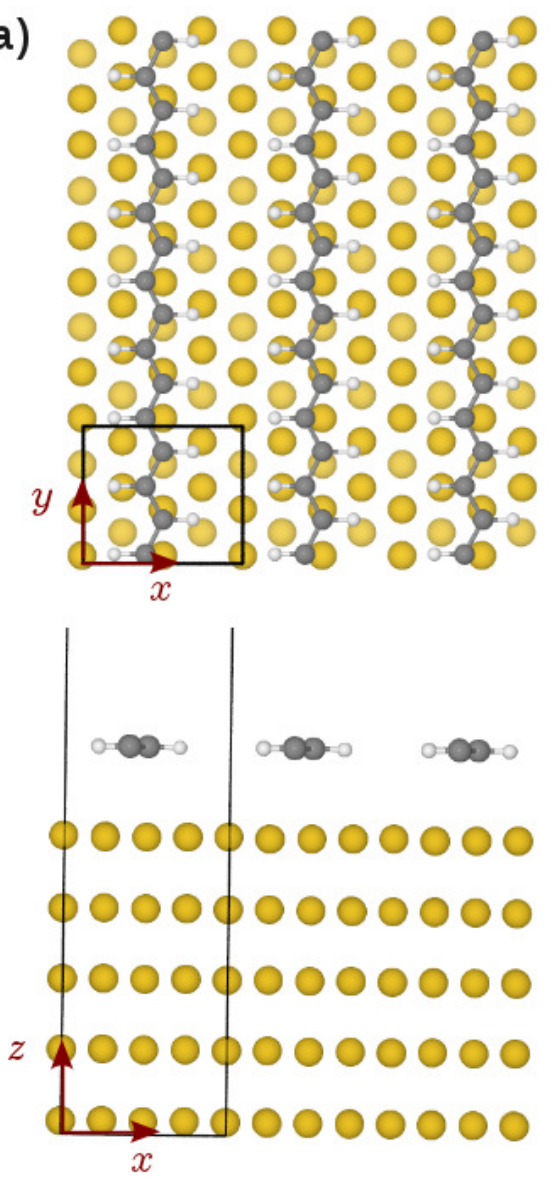

(b)
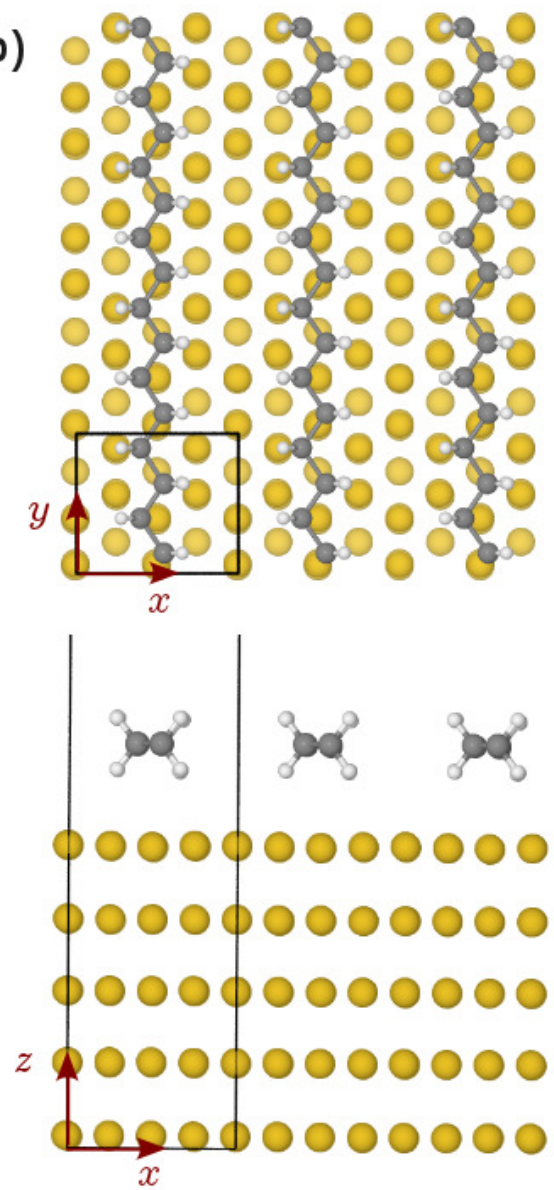

Figura 5.7: Representação esquemática da geometria de equilíbrio das interfaces (a) $\mathrm{TPA} / \mathrm{Au}(111)$ e (b) $\mathrm{PE} / \mathrm{Au}(111)$ obtidas com o funcional $\mathrm{PBE}+$ scvdW.

incluídos na aproximação ZORA para tratar os elétrons mais internos do Au, conforme já mencionado no início do capítulo.

Os principais parâmetros estruturais referentes a geometria de equilíbrio $\mathrm{PBE}+\mathrm{scvdW}$ dos polímeros adsorvidos sobre a superfície $\mathrm{Au}(111)$ são listados na tabela 5.6, e comparados com a geometria otimizada das cadeias isoladas. De acordo com estes resultados, a estrutura dos polímeros adsorvidos apresenta aparentemente apenas pequenos desvios em relação à geometria das cadeias isoladas caracterizando um processo de fisissorção. Notamos que a geometria planar do TPA e do PE é ligeiramente distorcida devido a interação com o metal. Entretanto, há uma mudança notável na geometria do polímero para a interface TPA/Au(111), no qual a cadeia polimérica perde a dimerização usual das ligações simples 
Tabela 5.5: Parâmetros estruturais (comprimentos de ligação e ângulos) referentes à geometria do estado fundamental do trans-poliacetileno (TPA) e polietileno (PE) em vácuo e adsorvidos sobre a superfície $\mathrm{Au}(111)$. Os cálculos foram realizados com o funcional $\mathrm{PBE}+$ scvdW.

\begin{tabular}{lcclll}
\hline & \multicolumn{2}{c}{ TPA } & & \multicolumn{2}{c}{ PE } \\
\cline { 2 - 3 } \cline { 5 - 6 } & isolado & adsorvido & & isolado & adsorvido \\
\hline$d_{C=C}$ & 1,395 & 1,401 & & \\
$d_{C-C}$ & 1,414 & 1,411 & & 1,508 & 1,507 \\
$d_{C-H}$ & 1,096 & 1,095 & & 1,104 & 1,105 \\
$\alpha_{C C C}$ & 125,6 & 125,3 & & 111,8 & 112,1 \\
$\gamma_{C C C C}$ & 180,0 & 179,6 & & 180,0 & 179,5 \\
$\gamma_{H C C H}$ & 180,0 & 178,7 & & 180,0 & 179,2 \\
\hline
\end{tabular}

e duplas após a adsorção. Este efeito indica uma possível transferência de carga entre os sistemas, visto que o TPA dopado com cargas adquire um caráter metálico com perda de dimerização.

Para determinar a distância de equilíbrio e energia de ligação dos polímeros adsorvidos sobre a superfície de ouro, realizamos uma série de cálculos de single-point considerando a geometria de equilíbrio $\mathrm{PBE}+\mathrm{scvdW}$ com todas as coordenadas atômicas do sistema fixas, variando apenas a distância vertical polímero superfície. Neste caso, o conjunto de base estendido tier 3 foi utilizado para todas as espécies atômicas para obter um alto nível de precisão no cálculo da energial total. Os resultados obtidos para a interface TPA/Au(111) com diferentes funcionais são mostrados na Figura 5.8. A exemplo do que tem sido observado em cálculos DFT anteriores [127] para interfaces molécula-metal, nossos resultados mostram que as interações vdW aumentam a energia de adsorção do TPA sobre a superfície Au(111). $\mathrm{O}$ valor obtido com o funcional $\mathrm{PBE}+\mathrm{scvdW}(0,79 \mathrm{eV})$ é aproximadamente $0,37 \mathrm{eV}$ maior que o LDA e 0,46 eV maior que o PBE. Esses valores são consistentes com os valores obtidos por cálculos DFT da literatura 119,128,129 para pequenas moléculas orgânicas adsorvidas sobre a superfície $\mathrm{Au}(111)$.

Uma quantidade especialmente importante para a descrição correta do acoplamento eletrônico polímero-metal é a separação vertical de equilíbrio entre os sub-sistemas da interface. Os pontos de mínimo das curvas de energia potencial versus distância mostrados na Fig. 5.8 indicam as distâncias $\mathrm{P} / \mathrm{M}$ de equilíbrio obtidas por diferentes funcionais. Notamos 


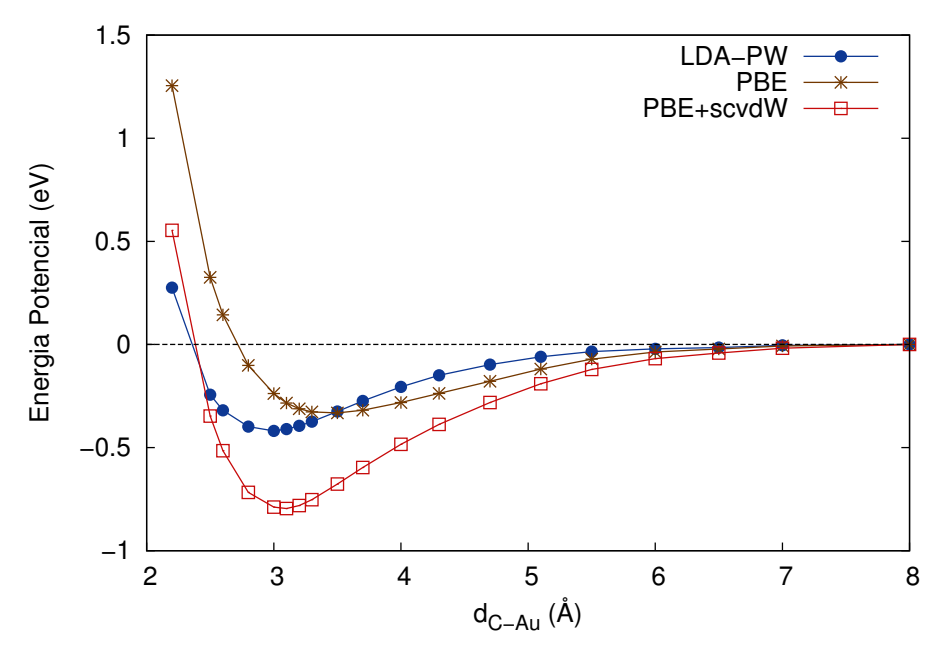

Figura 5.8: Energia de adsorção calculada para uma cadeia infinita de TPA sobre a superfície $\mathrm{Au}(111)$ como função da distância polímero-metal.

que as correções de dispersão adicionadas ao funcional PBE tendem a diminuir a distância polímero-metal resultando em uma maior interação na interface. A distância $\mathrm{P} / \mathrm{M}$ de equilíbrio calculada com $\mathrm{PBE}+\mathrm{scvdW}(3,18 \AA$ ) é cerca de $0,2 \AA$ maior que o valor LDA, e $0,3 \AA$ menor que o PBE. Cálculos DFT anteriores para a interface pentaceno/Au(111) 130, 131 resultam em uma distância ótima molécula-metal de $3,2 \AA$, enquanto cálculos PBE+scvdW para a interface benzeno/Au(111) 122 resultam em 3,05 A de separação vertical. Portanto, a distância de equilíbrio TPA-Au obtida por nossos cálculos PBE +scvdW está dentro do espectro de valores observados para outros sistemas molécula-ouro.

A curva de energia de adsorção versus distância calculada com o funcional $\mathrm{PBE}+\mathrm{scvdW}$ foi também determinada para a interface PE/ $\mathrm{Au}(111)$, e o resultado é mostrado na Fig. 5.9 juntamente com os valores obtidos para o TPA/Au(111). Primeiramente, vemos que a energia de ligação do PE com a superfície metálica $(0,75 \mathrm{eV})$ é bastante próxima do valor obtido para a interface $\mathrm{TPA} / \mathrm{Au}(111)$. Além disso, a distância de equilíbrio carbono-ouro calculada para a interface $\mathrm{PE} / \mathrm{Au}(111), 3,48 \AA$, é $0,3 \AA$ maior do que no caso do TPA, devido a interação entre os hidrogênios do PE e o substrato (ver Fig. 5.7). Esse resultado é consistente com os cálculos DFT-GGA reportados por Morikawa e colaboradores 132. 


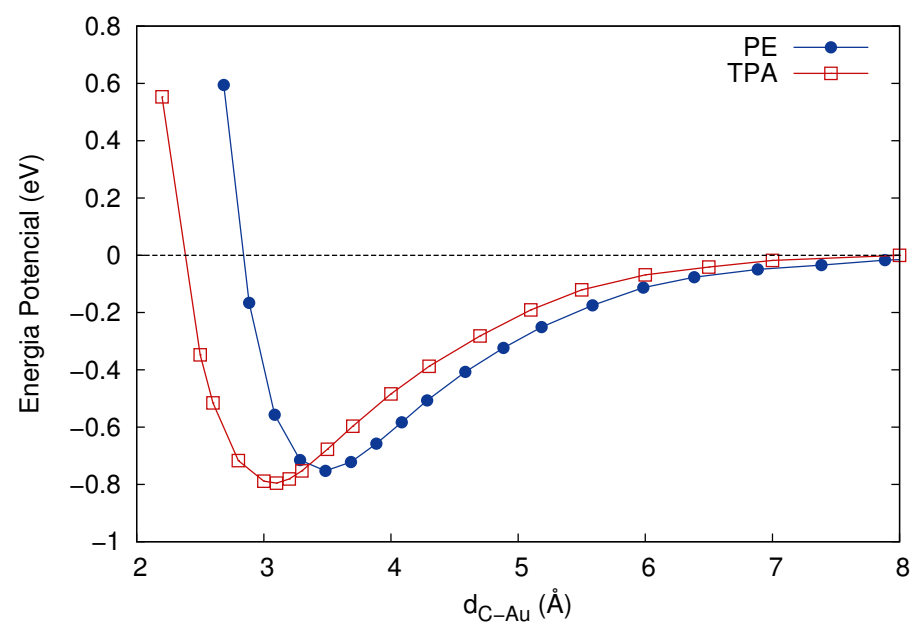

Figura 5.9: Energia potencial como função da distância vertical carbono-ouro cálculada para os polímeros PE e TPA adsorvidos sobre a superfície $\mathrm{Au}(111)$. Todos os cálculos foram realizados com o funcional $\mathrm{PBE}+\mathrm{scvdW}$ utilizando o conjunto de base tier 3 .

\subsubsection{Estrutura Eletrônica: Problemas da DFT}

Além das distorções na estrutura molecular, a adsorção de uma molécula conjugada ou polímero sobre uma superfície metálica pode induzir mudanças significativas nas propriedades eletrônicas do sistema orgânico, mesmo em um regime de interação fraca. Em geral, essas modificações se devem à redistribuição na densidade eletrônica do espaço real e/ou a hibridização de estados moleculares e metálicos [133]. O primeiro caso é comumente associado a modificações induzidas na função trabalho da superfície, enquanto o segundo resulta em mudanças na ocupação e alinhamento dos orbitais. Em ambos os casos, a intensidade da interação polímero-metal e, consequentemente das modificações induzidas na estrutura eletrônica depende da distância vertical entre os dois sistemas.

As mudanças observadas na estrutura eletrônica de interfaces híbridas OM são bastante sensíveis à natureza eletrônica do sistema orgânico adsorvido na superfície. Em particular, a posição relativa entre os níveis de fronteira (HOMO e LUMO) do sistema orgânico e o nível de Fermi do metal está estritamente relacionada aos processos de transferência de carga na interface. Nesta seção, apresentamos um estudo comparativo da estrutura eletrônica das interfaces $\mathrm{TPA} / \mathrm{Au}(111)$ e $\mathrm{PE} / \mathrm{Au}(111)$. 
Uma quantidade importante para avaliar os efeitos da interação polímero-metal é a variação da função trabalho, $\Delta \phi$, também chamada de deslocamento do nível de vácuo (vacuum level shift), que é dada pela expressão:

$$
\Delta \phi=\phi(\text { polimero } / \text { metal })-\phi(\text { metal })
$$

onde $\phi$ (polimero/metal) e $\phi($ metal $)$ correspondem à função trabalho do sistema híbrido PM e da superfície isolada do metal, respectivamente. Em geral, quanto maior a magnitude de $\Delta \phi$ mais forte é a interação entre o sistema orgânico e o substrato.

A modificação na função trabalho, $\Delta \phi$ para as interfaces TPA/Au(111) e PE/ $\mathrm{Au}(111)$ como função da distância vertical carbono-ouro é mostrada na Fig. 5.10. Para ambos os sistemas notamos que a magnitude de $\Delta \phi$ é negativa em todas as distâncias e aumenta gradualmente com a aproximação do polímero à superfice. A grandes distâncias, $\Delta \phi$ converge para um valor aproximadamente constante, sendo -0,02 eV no caso do PE, e -0,94 eV no caso do TPA.

Em torno das distâncias de equilíbrio $\mathrm{PBE}+\mathrm{scvdW}$ de cada interface, indicadas pelos limites inferior e superior da região sombreada da Fig. 5.10, o valor da função traballho da superfície de ouro modificada pela adsorção dos polímeros torna-se 4,36 eV no caso do TPA e 4,66 eV no caso do PE. Estes valores correspondem a uma diminuição de 0,9 eV e $0,6 \mathrm{eV}$, respectivamente, quando comparado a função trabalho experimental $(\phi=5,26 \mathrm{eV})$ da superfície limpa [118].

Cálculos DFT para moléculas aromáticas conjugadas tais como benzeno [134 e pentaceno [130 adsorvidas sobre a superfície $\mathrm{Au}(111)$ resultam em valores de $\Delta \phi$ em torno de -0,79 a -1,19 eV. No caso da interface com polímeros saturados [132 os cálculos DFT indicam uma diminuição de $0,7 \mathrm{eV}$ na função trabalho da superfície $\mathrm{Au}(111)$. Portanto, nossos resultados são consistentes com outros cálculos DFT realizados para sistemas molécula-metal com características eletrônicas similares as interfaces $\mathrm{P} / \mathrm{M}$ estudadas neste trabalho.

Existe porém uma diferença significativa entre os resultados para PE/Au e TPA/Au, já que o valor de $\Delta \phi$ permanece constante com os afastamento do polímero nesse último caso. Este efeito pode ser melhor entendido analisando a densidade de estados (DOS) dos sistemas interagentes $\mathrm{PE} / \mathrm{Au}$ e TPA/Au na geometria de equílibrio, comparadas à DOS dos sistemas isolados, conforme mostrado nas Figs. 5.12 e 5.11 . Iniciando pelo sistema $\mathrm{Pe} / \mathrm{Au}$, vemos que, conforme já mencionado acima, os estados eletrônicos ocupados do polímero estão abaixo do 


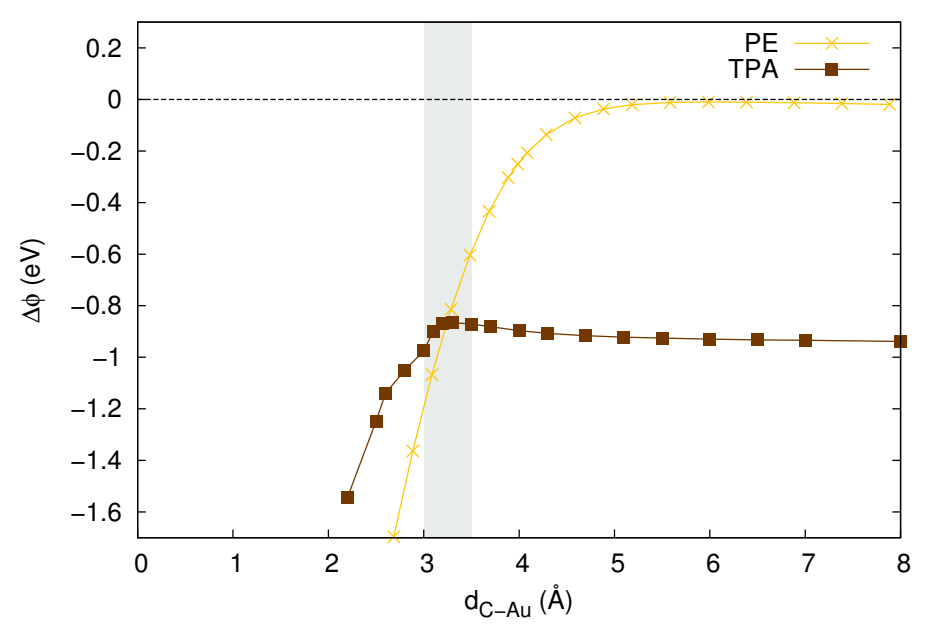

Figura 5.10: Variação na função trabalho, $\Delta \phi$, da superfície $A u(111)$ como função da distância vertical entre os plano dos carbonos da cadeia polimérica e o plano superior da superfície metálica. Os cálculos foram realizados com o funcional PBE utilizando o conjunto de base tier 3 .

nível de Fermi do metal, enquanto os desocupados estão muito acima - não há possibilidade de transferência de carga. Passando ao sistema TPA/Au, vemos que a PDOS do polímero adsorvido não apresenta gap de energia, indicando que o TPA passa a um estado metálico. De fato, o topo da banda de valência do TPA isolado obtido com PBE (ver Fig. 5.12(a)) está em -4,11 eV, portanto $1 \mathrm{eV}$ acima do nível de Fermi do metal calculado com o mesmo funcional. Assim, ao interagir com a superfície de ouro, o TPA deve perder elétrons para o metal se tornando positivamente carregado. De acordo com a análise populacional de Mulliken, a carga acumulada no TPA após a adsorção sobre a superfície de ouro é de 0,06e, o que é consistente com a transferência de cargas prevista com base na análise da DOS. Ainda na Fig. 5.12 (c) podemos notar uma perturbação nos picos localizados da PDOS do TPA (região em torno de $-5 \mathrm{eV}$ ) que indica uma possível hibridização desses estados com a banda $d$ do metal.

Em suma, vemos que o funcional PBE produz bons resultados para o sistema metálico. Por outro lado, conforme visto no capítulo 3, os autovalores DFT-KS subestimam as energias de ionização experimental de sistemas orgânicos resultando em uma descrição incorreta dos níveis eletrônicos de fronteira de polímeros conjugados tais como o TPA. Isto pode ter consequências drásticas no estudo de interfaces híbridas, como demonstrado acima para a 
Tabela 5.6: Valores teóricos para a distância vertical, $Z_{C}$, entre o sistema orgânico e o topo da superfície $\mathrm{Au}(111)$ e a variação da função trabalho, $\Delta \phi$, obtidos com o funcional PBE para os polímeros TPA e PE adsorvidos sobre a superfície $\mathrm{Au}(111)$. Resultados teóricos da literatura para as interfaces Benzeno/ $\mathrm{Au}(111)$ [134 e Pentaceno/Au(111) [131 são também incluídos para comparação.

\begin{tabular}{lcc}
\hline & $Z_{C} \AA$ & $\Delta \phi(\mathrm{eV})$ \\
\hline TPA & 3,18 & 0,90 \\
PE & 3,48 & 0,60 \\
Benzeno & 3,10 & 0,79 \\
Pentaceno & 3,20 & 1,19 \\
\hline
\end{tabular}

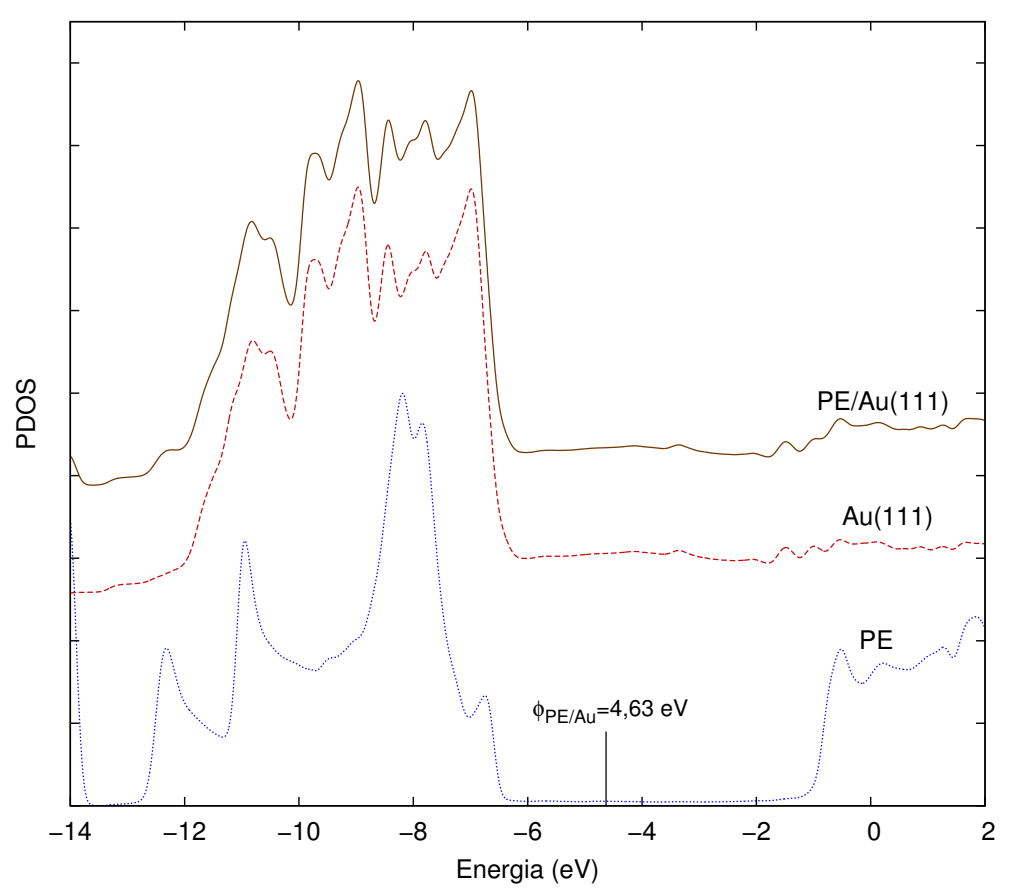

Figura 5.11: Densidade de estados projetada dos orbitais do PE sobre a superfície $\mathrm{Au}(111)$ calculada na distância de equilíbrio PBE + scvdW. Neste caso, os gráficos foram deslocados verticalmente para facilitar a análise. O cálculo foi realizado com o funcional PBE na base tier 2. 

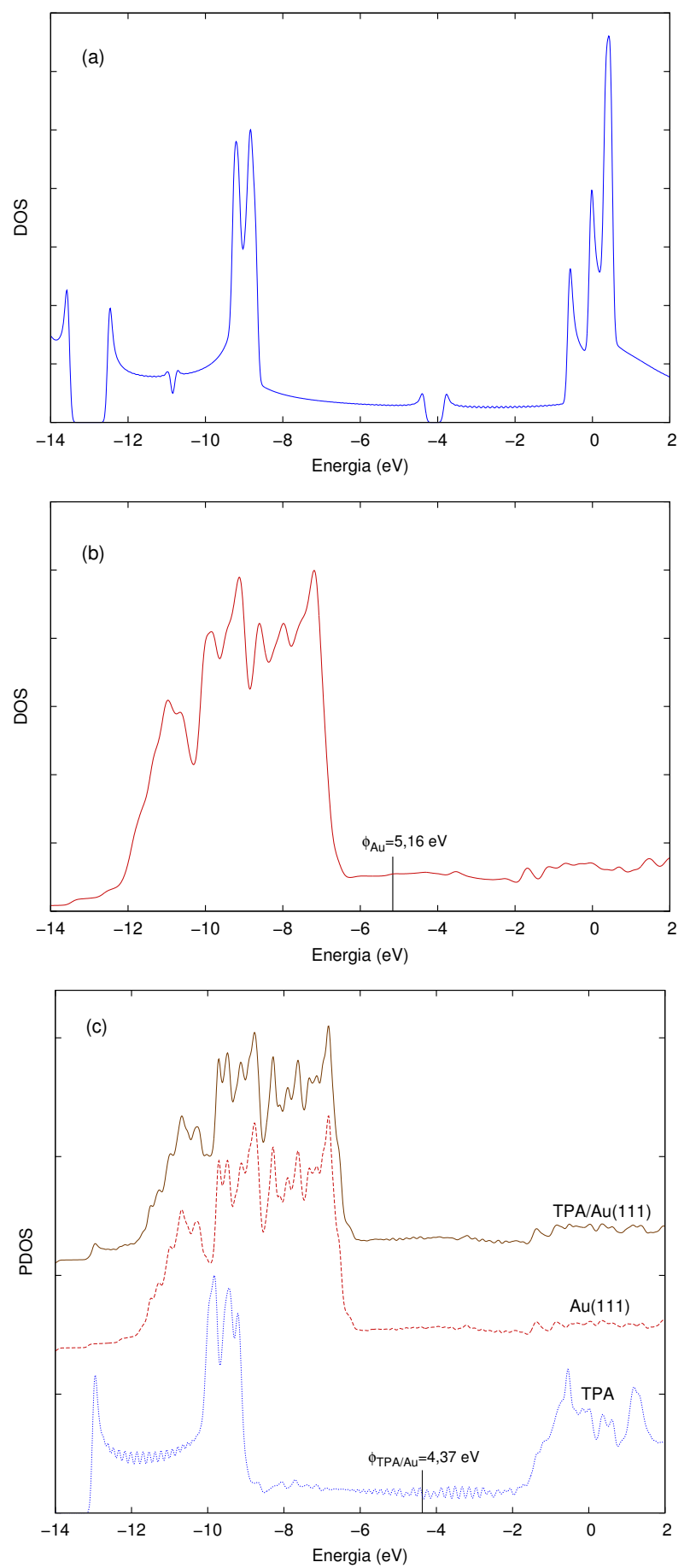

Figura 5.12: Densidade de estados total do (a) TPA e (b) do Au(111) isolados comparada a (c) densidade de estados projetada dos orbitais do TPA sobre a superfície $\mathrm{Au}(111)$ calculada na distância de equilíbrio PBE + scvdW. Neste caso, os gráficos foram deslocados verticalmente para facilitar a análise. Todos os cálculos foram realizados com o funcional PBE na base tier 2 . 
interface TPA/Au, no qual os cálculos DFT preveem uma transferência de carga artificial quando os dois subsistemas são colocados em contato formando a interface. Neste caso, ainda que o cálculo DFTh para interfaces híbridas polímero/metal fosse computacionalmente viável, o problema da transferência de carga não poderia ser resolvida com funcionais híbridos que adotem uma fração constante de troca exata, uma vez que cada subsistema requer diferentes valores de $\alpha$ para corrigir os níveis de energia. Nosso estudo deve ser assim tomado como um sinal de alerta quanto a necessidade de estratégias alternativas. 



\section{Capítulo 6}

\section{Conclusão}

Nesta tese realizamos um estudo teórico, baseado na Teoria do Funcional da Densidade, das propriedades eletrônicas de sistemas conjugados, focalizando propriedades intrinsecamente relevantes para dispositivos eletrônicos orgânicos, tais como potencial de ionização e afinidade eletrônica. Escolhemos para isso o trans-poliacetileno (TPA), o principal modelo para sistemas em conjugação $\pi$. Em seguida, estudamos a interação desses sistemas na configuração polímero/metal. Como sistemas modelo foram estudadas as interfaces de TPA/Au(111), e também como contraponto o polímero não conjugado polietileno $\mathrm{PE} / \mathrm{Au}(111)$.

Um importante desafio teórico enfrentado nesta Tese é a determinação precisa dos níveis eletrônicos de fronteira dos sistemas orgânicos dentro de um modelo de campo médio. Os problemas de métodos de campo médio na descrição de energias de ionização e gap fundamental são bem conhecidas, e vários procedimentos já foram propostos para superá-los. A passagem a métodos perturbativo de muitos corpos é um caminho confiável, entretanto a dependência com o ponto de partida é uma questão a ser considerada. Neste sentido, estudamos a dependência da correção de muitos corpos ou quasipartícula aplicada a cadeias de acetileno de diferentes comprimentos, e a partir de diferentes funcionais, e verificamos que a dependência com o comprimento é decididamente forte. De fato, a diferença no IP GW pode chegar a 2,0 eV dependendo do modelo de campo médio utilizado como ponto de partida, um resultado nunca antes visto na literatura. Estes resultados podem ser entendido da seguinte forma: uma vez que a DFT subestima o gap HOMO-LUMO, o uso de funções de Green construídas a partir desses orbitais para determinar a auto-energia GW resulta em 
uma superestimativa da blindagem. Já no caso do HF ocorre o oposto. Aqui adotamos uma estratégia baseada na aproximação $\mathrm{G}_{0} \mathrm{~W}_{0}$ para otimizar a fração de troca exata não-local (HF) do funcional híbrido PBEh. O procedimento consiste em variar a quantidade de troca exata do funcional PBEh até que o auto-valor do estado mais alto ocupado (HOMO) calculado com PBEh e com $\mathrm{G}_{0} \mathrm{~W}_{0} @ \mathrm{PBEh}$ sejam tão próximos quanto possível, levando portanto a uma consistência interna entre os dois modelos, sem um compromisso direto em reproduzir o experimento.

Iniciando a discussão por oligômeros de acetileno (OTA), nossos cálculos mostraram que o parâmetro de troca exata otimizado para cada molécula, $i c-\alpha$, apresenta uma dependência exponencial decrescente com o comprimento dos oligômeros, um efeito que está relacionado ao aumento da blindagem eletrônica em sistemas conjugados 1D. Este procedimento foi realizado para uma família de oligômeros de acetileno de tamanho variado e a fração ideal de troca exata obtida chega a $\sim 0,80$ para o oligômero mais curto, um valor bastante superior ao comumente adotado em funcionais híbridos tradicionais como PBE0 e B3LYP. Um passo adiante, a partir desses dados, estimamos a fração de troca exata ideal para uma cadeia conjugada 1D (TPA) em $\alpha \simeq 0.75$.

Através de comparações com dados experimentais disponíveis para os oligômeros menores, verificamos que o funcional híbrido otimizado para os sistemas orgânicos (ic-PBE) leva aos menores erros no cálculo do potencial de ionização dos OTAs. Além disso, a descrição do gap fundamental com o funcional ic-PBE é também bastante satisfatória devido à similaridade na distribuição espacial dos estados de fronteira HOMO e LUMO.

Em relação aos metais, investigamos a eficiência dos métodos DFT, incluindo os funcionais híbridos, na descrição das propriedades eletrônicas do ouro, representado na forma de bulk e superfície. Os modelos DFT convencionais (LDA e GGA), em geral, já fornecem uma boa descrição tanto das propriedades estruturais quanto eletrônicas dos sistemas metálicos. Nosso estudo do Au com o funcional PBE confirma esta qualidade. O efeito global da troca exata (neste caso, apenas $\alpha=0,25, \mathrm{PBE} 0$ ) sobre a estrutura de bandas do metal é promover uma abertura dos "gaps internos" e também um alargamento da banda de valência. O nível de Fermi é deslocado para energias mais baixas com o aumento da troca exata, e o espectro é distorcido em relação ao experimental, o que invalida o uso do mesmo funcional PBEh para metal e orgânico.

O estudo das interfaces polímero/metal foi realizado em um modelo periódico no qual o polímero depositado sobre um slab de metal é representado como uma cadeia infinita 
1D. Neste caso de interfaces, nosso trabalho destaca a necessidade de cuidado extremo no tratamento auto-consistente dos cálculos DFT, dada a disparidade do parâmetro ideal $\alpha$ para os dois componentes. 



\section{Apêndice A}

\section{Interações de van der Waals}

As interações de van der Waals ou forças de dispersão de London desempenham um papel determinante nas propriedades físicas de uma ampla variedade de sistemas, especialmente na descrição da morfologia de sólidos orgânicos [135, 136]. Estas interações são de natureza quântica e se originam da correlação eletrônica, que ocorrem devido a formação de interações instantâneas dipolo induzidas entre dois átomos polarizáveis. Classicamente, a interação de dispersão entre dois átomos (ou moléculas) separados por uma grande distância pode ser entendida como uma energia eletrostática atrativa do tipo $1 / R^{6}$ que surge quando um átomo induz um dipolo temporário em um segundo átomo a uma distância $R$. Em uma visão quântica, as forças de dispersão surgem de flutuações na densidade eletrônica que dão origem a dipolos instantâneos.

A Teoria do Funcional da Densidade dentro da aproximação de campo médio não trata adequadamente os efeitos de correlação de longo alcance existente em sistemas fracamente ligados [137]. A natureza semi-local dos funcionais de troca e correlação GGA levam a falhas severas na descrição do comportamento assintótico correto das forças dispersivas de vdW. Uma grande variedade de estratégias tem sido propostas para incluir as interações de vdW em cálculos DFT [138]. Aqui, faremos uma breve descrição do método proposto por Tkatchenko-Scheffler [57] (TS) para incluir os efeitos dispersivos de longo alcance (vdW) nos funcionais da DFT.

De acordo com o modelo TS-vdW, o termo $C_{6} / R^{6}$ é adicionado ao termo de energia internuclear, onde o coeficiente $C_{6}$ e o raio de van der Waals são determinados diretamente a partir da densidade eletrônica do estado fundamental DFT e valores de referência para 
átomos livres. Nesta aproximação, o coeficiente efetivo $C_{6 A A}^{e f}$ para um átomo na molécula é determinado a partir da relação:

$$
C_{6 A A}^{e f}=\frac{\eta_{A}^{e f}}{\eta_{A}^{l i v}}\left(\frac{\kappa_{A}^{l i v}}{\kappa_{A}^{e f}}\right)^{2}\left(\frac{V_{A}^{e f}}{V_{A}^{l i v}}\right)^{2} C_{6 A A}^{l i v} .
$$

sendo a constante de proporcionalidade $\left(\eta_{A}^{e f} / \eta_{A}^{l i v}\right)\left(\kappa_{A}^{l i v} / \kappa_{A}^{e f}\right)^{2}$ tomada igual a um por simplicidade. A relação entre o volume efetivo do átomo na molécula e o volume do átomo livre é obtido utilizando o esquema de partição de Hirshfeld para a densidade eletrônica,

$$
\begin{gathered}
\frac{V_{A}^{e f}}{V_{A}^{l i v}}=\left(\frac{\int r^{3} w_{a}(\mathbf{r}) n(\mathbf{r}) d^{3} \mathbf{r}}{\int r^{3} n_{A}^{l i v}(\mathbf{r}) d^{3} \mathbf{r}}\right) \\
w_{a}(\mathbf{r})=\frac{n_{A}^{l i v}}{\sum n_{B}^{l i v}(\mathbf{r})}
\end{gathered}
$$

Já, $w_{a}(\mathbf{r})$ é o peso da contribuição do átomo A à densidade molecular em $r$. Os termos $n(\mathbf{r})$ e $n_{A}^{\text {liv }}(\mathbf{r})$ na eq. A.3 correspondem as densidades eletrônicas totais, da molécula e do átomo livre A, respectivamente, que são determinadas através de cálculos DFT. As interações de vdW são relevantes para interações atômicas seja intra ou inter-moleculares. Uma vez que os coeficientes $C_{6}$ são aditivos, o coeficiente da interação intermolecular, $C_{6}^{\text {mol }}$, é dado pela soma de todas as contribuições interatômicas

$$
C_{6}^{m o l}=\sum_{A \in M_{1}} \sum_{B \in M_{2}} C_{6 A B}^{e f}
$$

onde os índices $M_{1}$ e $M_{2}$ correspondem à primeira e à segunda molécula do sistema, respectivamente.

Após determinar os coeficientes da interação de van der Waals, $C_{6 A B}$, a energia total do sistema, obtida por um cálculo DFT, é corrigida adicionando o termo

$$
E_{v d W}=-\frac{1}{2} \sum_{A, B} f_{d a m p}\left(R_{A B}, R_{A}^{0}, R_{B}^{0}\right) C_{6 A B} R_{A B}^{-6},
$$


onde $R_{A B}$ é a distância entre os átomos $\mathrm{A}$ e $\mathrm{B}$, e $R_{A}^{0}, R_{B}^{0}$ são os respectivos raios de van der Waals. Utilizando a definição de volume atômico efetivo dado na eq. A.2 o raio vdW de um átomo na molécula torna-se:

$$
R_{e f}^{0}=\left(\frac{V_{A}^{e f}}{V_{A}^{l i v}}\right)^{1 / 3} R_{\text {liv }}^{0}
$$

O $R_{\text {liv }}^{0}$ é então definido para o conjunto de átomos da tabela periódica a partir do valor de contorno da densidade eletrônica correpondente ao raio vdW de átomos livres esfericamente simétricos (gases nobres). Por fim, o esquema TS para correções de vdW propõe uma função "damping" do tipo Fermi,

$$
f_{\text {damp }}\left(R_{A B}, R_{A B}^{0}\right)=\left\{1+\exp \left[-d\left(\frac{R_{A B}}{s_{R} R_{A B}^{0}}-1\right)\right]\right\}^{-1}
$$

onde $R_{A B}^{0}=R_{A}^{0}+R_{B}^{0}$. O parâmetro $d$ ajusta a declividade da função "damping", e o coeficiente $s_{R}$ é o único parâmetro empírico da função "damping", que determina o "onset" da correção vdW para um funcional xc específico em termos da distância.

O esquema TS-vdW proposto para incluir os efeitos de dispersão nos funcionais LDA e GGA tem sido utilizado com sucesso na descrição de interações não-ligadas em uma ampla variedade sistemas moleculares [139, 140]. Em particular, a simplicidade e eficiência do formalismo envolvido na determinação do coeficiente $C_{6}$ e da correção de energia $E_{v d W}$ associada torna o esquema TS-vdW bastante útil para o estudo dos sistemas de interesse em noso projeto, conforme será discutido no capítulo de resultados. 



\section{Apêndice B}

\section{O pacote computacional "FHI-aims"}

O tema central do nosso projeto é o estudo teórico da formação da interface metalpolímero, considerando os aspectos morfológicos da estrutura supramolecular bem como a descrição precisa dos estados eletrônicos a nível atomístico. Isso requer o uso de uma metodologia multi-escala e multi-técnica que inclui modelos clássicos para o estudo da morfologia, seguido por cálculos de primeiros princípios para o tratamento das propriedades eletrônicas. No entanto, a escala de tamanho dos sistemas de interesse juntamente com a necessidade de uma descrição precisa dos níveis eletrônicos nas fronteiras de banda restringe as possibilidades de uso de algorítmos computacionais eficientes para os cálculos de estrutura eletrônica. Nesse aspecto, o programa "FHI-AIMS" (The Ab initio Molecular Simulaions Package) [51] desenvolvido recentemente no Fritz Haber Institute de Berlim tem se mostrado uma alternativa viável para suprir algumas necessidades computacionais do nosso projeto, por incorporar uma série de características importantes que preenche os requisitos básicos para o desenvolvimento deste trabalho:

- é um pacote DFT de primeiros princípios e incorpora tanto a aproximação LDA quanto diferentes GGAs;

- permite o estudo tanto de sistemas finitos $(v(\mathbf{r}) \rightarrow 0$ quando $r \rightarrow \infty)$ quanto de sistemas periódicos;

- para tal, utiliza um conjunto de base numérico centrado em átomos, sistematicamente desenvolvido para todos os elementos da tabela periódica; 
- tratamento all-electron - inclui explicitamente todos os elétrons do sistema, com correções relativísticas para átomos pesados;

- estão implementados métodos pós-DFT - funcionais híbridos (B3LYP, PBE0, HSE...), e MP2, RPA e GW quanto ao tratamento de muitos corpos para sistemas finitos;

- alto nível de paralelização.

Uma característica particularmente importante que distingue o "FHI-aims" dos demais pacotes computacionais atualmente diponíveis para cálculos de estrutura eletrônica é a forma funcional do conjunto de base empregado nos cálculos. A seguir, descreveremos brevemente a estrutura do conjunto de base implementao no "FHI-aims" e que será utilizado ao longo deste trabalho.

\section{Conjunto de base numérico centrado em átomos}

A escolha particular das funções de base de orbitais atômicos numéricos (OANs) é ponto chave para a precisão e eficiência nos cálculos de estrutura eletrônica realizados com o "FHI-AIMS". Segundo a idéia originalmente proposta, a densidade eletrônica na aproximação de KS é representada no espaço de funções de base localizada centrada em átomos, pelo produto de funções $\varphi_{i}(r)$, com a seguinte forma funcional:

$$
\varphi_{i}(r)=\frac{u(r)}{r} Y_{l m}(\Omega)
$$

A forma radial das funções $\varphi_{i}(r)$ é determinada numericamente,

$$
\left[-\frac{1}{2} \frac{\mathrm{d}^{2}}{\mathrm{~d} r^{2}}+\frac{l(l+1)}{r^{2}}+\nu_{i}(r)+\nu_{c u t}(r)\right] u_{i}(r)=\varepsilon_{i} u_{i}(r)
$$

onde $\nu_{i}(r)$ é o potencial que define o comportamento principal de $u_{i}(r)$, e $\nu_{\text {cut }}(r)$ é um potencial de confinamento que assegura o decaimento suave das funções radiais para zero. Em particular, a eq. B.2 permite incluir uma base mínima que consiste das funções de "caroço" e valência de átomos livres, esfericamente simétricas, simplesmente ajustando $\nu_{i}(r)$ ao potencial radial auto-consistente do átomo livre. Esta base mínima facilita bastante o tratamento all-electron, visto que ele naturalmente leva em conta as oscilações da função de onda próximas do núcleo. 
O conjunto de base mínimo pode ser aumentado com funções de polarização de maior momento angular, as quais são apropriadamente escolhidas a partir de várias funções radiais do tipo atômico, hidrogenóides ou iônicas, com o potencial de confinamento $\nu_{\text {cut }}(r)$ variável. As funções de base polarizadas são tipicamente agrupadas em subconjuntos de funções com diferentes momento angulares, spd, spdf..., e são assim organizadas em camadas ou "tiers" na linguagem FHI-aims, no qual a base minima corresponde ao "tier 1". A tabela B.1 resume os parâmetros das funções radiais ótimas para átomos de carbono e hidrogênio que serão utilizadas como conjuntos de base ao longo deste trabalho.

Tabela B.1: Funções radiais ótimas para os átomos de hidrogênio $(\mathrm{H})$ e carbono $(\mathrm{C})$ utilizadas como conjunto de base OANs no código "FHI-aims". A primeira linha representa as funções radiais dos átomos livres (configuração de gás nobre e números quânticos referentes as funções radiais de valência adicionais). "H( $n l, z)$ " denota as funções de base hidrogenóides para o potencial Coulombiano $z / r$, incluindo os números quânticos radial e de momento angular, $n$ e $l$.

\begin{tabular}{ccc}
\hline \hline & $\mathrm{H}$ & $\mathrm{C}$ \\
\hline & $1 \mathrm{~s}$ & {$[\mathrm{He}]+2 \mathrm{~s} 2 \mathrm{p}$} \\
& $\mathrm{H}(2 \mathrm{~s}, 2.1)$ & $\mathrm{H}(2 \mathrm{p}, 1.7)$ \\
tier 1 & $\mathrm{H}(2 \mathrm{p}, 3.5)$ & $\mathrm{H}(3 \mathrm{~d}, 6.0)$ \\
& - & $\mathrm{H}(2 \mathrm{~s}, 4.9)$ \\
\hline & $\mathrm{H}(1 \mathrm{~s}, 0.85)$ & $\mathrm{H}(4 \mathrm{f}, 9.8)$ \\
& $\mathrm{H}(2 \mathrm{p}, 3.7)$ & $\mathrm{H}(3 \mathrm{p}, 5.2)$ \\
tier 2 & $\mathrm{H}(2 \mathrm{~s}, 1.2)$ & $\mathrm{H}(3 \mathrm{~s}, 4.3)$ \\
& $\mathrm{H}(3 \mathrm{~d}, 7.0)$ & $\mathrm{H}(5 \mathrm{~g}, 14.4)$ \\
& - & $\mathrm{H}(3 \mathrm{~d}, 6.2)$ \\
\hline & $\mathrm{H}(4 \mathrm{f}, 11.2)$ & $\mathrm{H}(2 \mathrm{p}, 5.6)$ \\
tier 3 & $\mathrm{H}(3 \mathrm{p}, 4.8)$ & $\mathrm{H}(2 \mathrm{~s}, 1.4)$ \\
& $\mathrm{H}(4 \mathrm{~d}, 9.0)$ & $\mathrm{H}(3 \mathrm{~d}, 4.9)$ \\
& $\mathrm{H}(3 \mathrm{~s}, 3.2)$ & $\mathrm{H}(4 \mathrm{f}, 11.2)$ \\
\hline & & $\vdots$ \\
\hline \hline
\end{tabular}

Contrariamente ao caso das ondas planas, as funções de base OANs possuem uma série de parâmetros ajustáveis que influenciam o grau de convergência da base e a eficiência computacional. O "FHI-aims" disponibiliza, portanto, uma hierarquia de parâmetros prédefinidos do conjunto de base OAN para todos os elementos da tabela periódica designados por: "light", "tight" e "really tight". Estes são ordenados em uma sequência progressivamente 
mais fina para a malha na qual os elementos de matriz da Hamiltoniana são numericamente integrados. O conjunto de base "light" utiliza como padrão as funções radiais da base mínima "tier 1", com um raio de corte de 3,5 A para estas funções. Já as bases "tight" e "really tight" são iguais entre si com respeito ao número de funções radiais ("tier 2" por padrão), e diferem apenas com respeito ao momento ângular máximo ( $l_{\text {Hartree }}=6$ para "tight" e $l_{\text {Hartree }}=8$ para "really tight") utilizado na expansão da densidade eletrônica e a partir do qual o potencial de Hartree é construído. Uma descrição mais completa e detalhada do processo iterativo de construção das bases OANs no "FHI-AIMS" pode ser encontrada nas referências [51, 141].

Os três níveis de conjuntos de base discutidos acima foram exaustivamente testados para diversos sistemas, incluindo moléculas, sólidos e superfícies [139, 140], e aglomerados de átomos [142,143. Ressaltamos que polímeros conjugados de interesse ao nosso projeto não foram ainda estudados com o "FHI-AIMS", e dependendo das propriedades de interesse funções de base adicionais podem ser necessárias. Portanto, iniciamos o nosso estudo com uma análise cuidadosa da aplicabilidade dos conjuntos de base do "FHI-aims", conforme será apresentado a seguir. 


\section{Referências Bibliográficas}

[1] X. Guo, M. Baumgarten, e K. Mullen, "Designing $\pi$-conjugated polymers for organic electronics," Prog. Poli. Sci. 38 (2013) $1832-1908$.

[2] L. Akcelrud, "Electroluminescent polymers," Prog. Poli. Sci. 28 (2003) 875.

[3] M. Scharber e N. Sariciftci, "Efficiency of bulk-heterojunction organic solar cells," Prog. Poli. Sci. 38 (2013) 1929 - 1940. Topical issue on Conductive Polymers.

[4] C. B. Nielsen e I. McCulloch, "Recent advances in transistor performance of polythiophenes," Prog. Poli. Sci. 38 (2013) 2053 - 2069. Topical issue on Conductive Polymers.

[5] F. Liu, Y. Gu, X. Shen, S. Ferdous, H.-W. Wang, e T. P. Russell, "Characterization of the morphology of solution-processed bulk heterojunction organic photovoltaics," Prog. Poli. Sci. 38 (2013) 1990 - 2052. Topical issue on Conductive Polymers.

[6] S. C. Veenstra e H. T. Jonkman, "Energy-level alignment at metal-organic and organic-organic interfaces," Journal of Polymer Science Part B: Polymer Physics $4 \mathbf{1}$ (2003), no. 21, 2549-2560.

[7] M. Fahlman, A. Crispin, X. Crispin, S. K. M. Henze, M. P. de Jong, W. Osikowicz, C. Tengstedt, e W. R. Salaneck, "Electronic structure of hybrid interfaces for polymer-based electronics.," J. Phys. Condens. Matter. 19 (2007) 183202.

[8] S. Braun, W. R. Salaneck, e M. Fahlman, "Energy-level alignment at organic/metal and organic/ organic interfaces," Adv. Mater. 21 (2009) 1450.

[9] W. P. Su, J. R. Schrieffer, e A. J. Heeger, "Solitons in polyacetylene," Phys. Rev. Lett. 42 (1979) 1698-1701. 
[10] D. S. Galvão, D. A. dos Santos, B. Laks, C. P. de Melo, e M. J. Caldas, "Role of disorder in the conduction mechanism in polyanilines," Phys. Rev. Lett. 63 (1989) $786-789$.

[11] M. Springborg, "Self-consistent electronic structures of polyacetylene," Phys. Rev. B 33 (1986) 8475-8489.

[12] M. Rohlfing e S. G. Louie, "Optical excitations in conjugated polymers," Phys. Rev. Lett. 82 (1999) 1959-1962.

[13] K. Burke, "Perspective on density functional theory," J. Chem. Phys. 136 (2012) 150901.

[14] J. C. Slater, "A simplification of the Hartree-Fock method," Phys. Rev. 81 (1951) 385-390.

[15] D. R. Hartree, "The Wave Mechanics of an Atom with a Non-Coulomb Central Field. Part I. Theory and Methods," Mathematical Proceedings of the Cambridge Philosophical Society 24 (2008) 89.

[16] V. Fock, "Näherungsmethode zur Losung des quantenmechanischen Mehrkörperprobleme," Z. Phys 61 (1930) 126-148.

[17] P. S. Bagus, "Self-consistent-field wave functions for hole states of some Ne-like and Ar-like ions," Phys. Rev. 139 (1965) A619-A634.

[18] G. de Oliveira, J. M. L. Martin, F. de Proft, e P. Geerlings, "Electron affinities of the first- and second-row atoms: Benchmark ab initio and density-functional calculations," Phys. Rev. A 60 (1999) 1034-1045.

[19] V. Lemierre, A. Chrostowska, A. Dargelos, e H. Chermette, "Calculation of ionization potentials of small molecules: a comparative study of different methods.," J. Phys. Chem. A 109 (2005) 8348-55.

[20] T. Koopmans, "Ãœber die zuordnung von wellenfunktionen und eigenwerten zu den einzelnen elektronen eines atoms," Physica 1 (1934), no. 1-6, $104-113$. 
[21] P. Politzer e F. Abu-Awwad, "A comparative analysis of Hartree-Fock and Kohn-Sham orbital energies," Theor. Chem. Acc. 99 (1998) 83-87.

[22] S. Hamel, P. Duffy, M. E. Casida, e D. R. Salahub, "Kohn-Sham orbitals and orbital energies: fictitious constructs but good approximations all the same," Journal of Electron Spectroscopy and Related Phenomena 123 (2002) 345 - 363.

[23] W. Kohn, a. D. Becke, e R. G. Parr, "Density Functional Theory of Electronic Structure," J. Phys. Chem. 100 (1996) 12974-12980.

[24] W. Kohn e L. J. Sham, "Self-consistent equations including exchange and correlation effects," Phys. Rev. 140 (1965) A1133-A1138.

[25] P. Hohenberg e W. Kohn, "Inhomogeneous electron gas," Phys. Rev. 136 (1964) B864-B871.

[26] D. M. Ceperley e B. J. Alder, "Ground state of the electron gas by a stochastic method," Phys. Rev. Lett. 45 (1980) 566-569.

[27] J. P. Perdew e A. Zunger, "Self-interaction correction to density-functional approximations for many-electron systems," Phys. Rev. B 23 (1981) 5048-5079.

[28] J. P. Perdew e Y. Wang, "Accurate and simple analytic representation of the electron-gas correlation energy," Phys. Rev. B 45 (1992) 13244-13249.

[29] V. N. Staroverov, G. E. Scuseria, J. Tao, e J. P. Perdew, "Tests of a ladder of density functionals for bulk solids and surfaces," Phys. Rev. B 69 (2004) 075102.

[30] G. I. Csonka, J. P. Perdew, A. Ruzsinszky, P. H. T. Philipsen, S. Lebègue, J. Paier, O. A. Vydrov, e J. G. Ángyán, "Assessing the performance of recent density functionals for bulk solids," Phys. Rev. B 79 (2009) 155107.

[31] J. P. Perdew, K. Burke, e M. Ernzerhof, "Generalized gradient approximation made simple," Phys. Rev. Lett. 77 (1996) 3865-3868.

[32] S. F. Sousa, P. A. Fernandes, e M. J. Ramos, "General Performance of Density Functionals," J. Phys. Chem. A 111 (2007) 10439-10452. 
[33] M. Ernzerhof e G. E. Scuseria, "Assessment of the Perdew-Burke-Ernzerhof exchange-correlation functional," J. Chem. Phys. 110 (1999) 5029-5036.

[34] C. Adamo e V. Barone, "Toward reliable density functional methods without adjustable parameters: The PBE0 model," J. Phys. Chem. 110 (1999) 6158.

[35] J. P. Perdew, M. Ernzerhof, e K. Burke, "Rationale for mixing exact exchange with density functional approximations," J. Chem. Phys. 105 (1996) 9982.

[36] A. D. Becke, "Density-functional thermochemistry. iii. the role of exact exchange," J. Chem. Phys. 98 (1993) 5648-5652.

[37] K. Kim e K. D. Jordan, "Comparison of density functional and MP2 calculations on the water monomer and dimer," J. Phys. Chem. 98 (1994) 10089-10094.

[38] P. J. Stephens, F. J. Devlin, C. F. Chabalowski, e M. J. Frisch, "Ab initio calculation of vibrational absorption and circular dichroism spectra using density functional force fields," J. Phys. Chem. 98 (1994) 11623-11627.

[39] A. D. Becke, "Density-functional exchange-energy approximation with correct asymptotic behavior," Phys. Rev. A 38 (1988) 3098-3100.

[40] S. H. Vosko, L. Wilk, e M. Nusair, "Accurate spin-dependent electron liquid correlation energies for local spin density calculations: a critical analysis," Can. J. Phys. 58 (1980) 1200-1211.

[41] C. Lee, W. Yang, e R. G. Parr, "Development of the Colle-Salvetti correlation-energy formula into a functional of the electron density," Phys. Rev. B 37 (1988) 785-789.

[42] G. Brocks, "Density functional study of polythiophene derivatives," J. Phys. Chem. 100 (1996) 17327-17333.

[43] G. Zheng, S. J. Clark, S. Brand, e R. A. Abram, "First-principles studies of the structural and electronic properties of poly-para-phenylene vinylene," J. Phys.: Condens. Matter 16 (2004) 8609-8620.

[44] P. Mori-Sánchez, A. J. Cohen, e W. Yang, "Localization and delocalization errors in density functional theory and implications for band-gap prediction," Phys. Rev. Lett. 100 (2008) 146401. 
[45] L. D. Landau Sov. Phys.-JETP 3 (1957) 920.

[46] L. D. Landau Sov. Phys.-JETP 5 (1957) 101.

[47] L. Hedin, "New method for calculating the one-particle Green's function with application to the electron gas problem," Phys. Rev. 139 (1965) A796-A823.

[48] P. Rinke, A. Qteish, J. Neugebauer, C. Freysoldt, e M. Scheffler, "Combining GW calculations with exact-exchange density-functional theory: an analysis of valence-band photoemission for compound semiconductors," New. J. Phys. 7 (2005) 126-126.

[49] F. Fuchs, J. Furthmüller, F. Bechstedt, M. Shishkin, e G. Kresse, "Quasiparticle band structure based on a generalized Kohn-Sham scheme," Phys. Rev. B 76 (2007) 115109 .

[50] F. Bruneval e M. A. L. Marques, "Benchmarking the Starting Points of the GW Approximation for Molecules," J. Chem. Theory Comput. 9 (2013) 324-329.

[51] V. Blum, R. Gehrke, F. Hanke, P. Havu, V. Havu, X. Ren, K. Reuter, e M. Scheffler, "Ab initio molecular simulations with numeric atom-centered orbitals," Comput. Phys. Commun. 180 (2009) 2175-2196.

[52] S. Rohra, E. Engel, e A. Görling, "Exact-exchange Kohn-Sham formalism applied to one-dimensional periodic electronic systems," Phys. Rev. B 74 (2006) 1-9.

[53] M. L. Tiago, M. Rohlfing, e S. G. Louie, "Bound excitons and optical properties of bulk trans -polyacetylene," Phys. Rev. B 70 (2004) 193204.

[54] S. Hirata, H. Torii, e M. Tasumi, "Density-functional crystal orbital study on the structures and energetics of polyacetylene isomers," Phys. Rev. B 57 (1998) 11994-12001.

[55] W. R. Salaneck, H. R. Thomas, C. B. Duke, A. Paton, E. W. Plummer, A. J. Heeger, e A. G. MacDiarmid, "Photoelectron spectra of $\mathrm{AsF}_{5}$-doped polyacetylenes," J. Chem. Phys. 71 (1979) 2044-2050. 


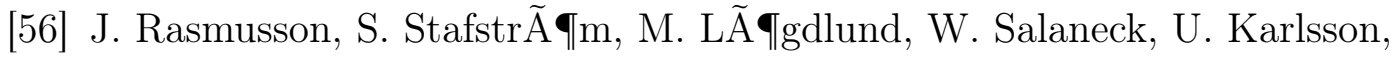
D. Swanson, A. Macdiarmid, e G. Arbuckle, "Photoelectron studies of trans-polyacetylene using synchrotron radiation," Synthetic Metals 41 (1991) 1365 1368.

[57] A. Tkatchenko e M. Scheffler, "Accurate molecular van der Waals interactions from ground-state electron density and free-atom reference data," Phys. Rev. Lett. 102 (2009) 073005.

[58] C. R. Fincher, C. E. Chen, A. J. Heeger, A. G. MacDiarmid, e J. B. Hastings, "Structural determination of the symmetry-breaking parameter in trans $-(\mathrm{CH})_{x}$," Phys. Rev. Lett. 48 (1982) 100-104.

[59] P. A. Limacher, K. V. Mikkelsen, e H. P. Lüthi, "On the accurate calculation of polarizabilities and second hyperpolarizabilities of polyacetylene oligomer chains using the CAM-B3LYP density functional.," J. Chem. Phys. 130 (2009) 194114.

[60] V. Lacivita, M. Reèrat, R. Orlando, M. Ferrero, e R. Dovesi, "Calculation of longitudinal polarizability and second hyperpolarizability of polyacetylene with the coupled perturbed Hartree-Fock/Kohn-Sham scheme: Where it is shown how finite oligomer chains tend to the infinite periodic polymer.," J. Chem. Phys. 136 (2012) 114101.

[61] C. S. Yannoni e T. C. Clarke, "Molecular geometry of cis - and trans -polyacetylene by nutation nmr spectroscopy," Phys. Rev. Lett. 51 (1983) 1191-1193.

[62] C. R. Fincher, M. Ozaki, M. Tanaka, D. Peebles, L. Lauchlan, A. J. Heeger, e A. G. MacDiarmid, "Electronic structure of polyacetylene: Optical and infrared studies of undoped semiconducting $\left(\mathrm{ch}_{x}\right)$ and heavily doped metallic $\left(\mathrm{ch}_{x}\right), "$ Phys. Rev. B 20 (1979) 1589-1602.

[63] G. Leising, "Anisotropy of the optical constants of pure and metallic polyacetylene," Phys. Rev. B 38 (1988) 10313-10322.

[64] Y.-W. Chang e B.-Y. Jin, "Correlation effects of $\pi$ electrons on the band structures of conjugated polymers using the self-consistent GW approximation with vertex corrections.," J. Chem. Phys. 136 (2012) 024110. 
[65] U. Salzner, P. G. Pickup, R. A. Poirier, e J. B. Lagowski, "Accurate Method for Obtaining Band Gaps in Conducting Polymers Using a DFT/Hybrid Approach," J. Phys. Chem. A 102 (1998) 2572-2578.

[66] N. Sai, P. Barbara, e K. Leung, "Hole Localization in Molecular Crystals from Hybrid Density Functional Theory," Phys. Rev. Lett. 106 (2011) 1-4.

[67] U. Salzner, J. B. Lagowski, P. G. Pickup, e R. A. Poirier, "Design of low band gap polymers employing density functional theory-hybrid functionals ameliorate band gap problem," J. Comput. Chem. 18 (1997) 1943-1953.

[68] K. Kimura, Handbook of HeI photoelectron spectra of fundamental organic molecules: ionization energies, ab initio assignments, and valence electronic structure for 200 molecules. Japan Scientific Societies Press, 1981.

[69] M. Beez, G. Bieri, H. Bock, e E. Heilbronner, "The ionization potentials of butadiene, hexatriene, and their methyl derivatives: Evidence for through space interaction between double bond $\pi$-orbitals and non-bonded pseudo- $\pi$ orbitals of methyl groups?," Helvetica Chim. Acta 56 (1973) 1028-1046.

[70] T. Bally, S. Nitsche, K. Roth, e E. Haselbach, "Excited states of polyene radical cations: limitations of koopmans' theorem," J. Am. Chem. Soc. 106 (1984) 3927-3933.

[71] P. Rademacher, K. Kowski, H. Hopf, D. Klein, O. Klein, e C. Suhrada, "Photoelectron spectra and electronic structures of highly substituted polyenes," J. Mol. Struct. 567-568 (2001) $11-18$.

[72] X.-B. Li, H.-Y. Wang, X.-D. Yang, Z.-H. Zhu, e Y.-J. Tang, "Size dependence of the structures and energetic and electronic properties of gold clusters.," J. Chem. Phys. 126 (2007) 084505.

[73] M. L. Tiago, P. R. C. Kent, R. Q. Hood, e F. a. Reboredo, "Neutral and charged excitations in carbon fullerenes from first-principles many-body theories.," J. Chem. Phys. 129 (2008) 084311. 
[74] N. Marom, X. Ren, J. E. Moussa, J. R. Chelikowsky, e L. Kronik, "Electronic structure of copper phthalocyanine from $\mathrm{G}_{0} \mathrm{~W}_{0}$ calculations," Phys. Rev. B 84 (2011) 195143.

[75] U. Salzner, "Modeling photoelectron spectra of conjugated oligomers with time-dependent density functional theory.," J. Phys. Chem. A 114 (2010) 10997-1007.

[76] U. Salzner e A. Aydin, "Improved Prediction of Properties of $\pi$-Conjugated Oligomers with Range-Separated Hybrid Density Functionals," J. Chem. Theory Comput. 7 (2011) 2568-2583.

[77] V. Atalla, M. Yoon, F. Caruso, P. Rinke, , e M. Scheffler, "Hybrid density functional theory meets quasiparticle calculations: a consistent electronic structure approach," Phys. Rev. B 88 (2013) 165122.

[78] M. Levy, J. P. Perdew, e V. Sahni, "Exact differential equation for the density and ionization energy of a many-particle system," Phys. Rev. A 30 (1984) 2745-2748.

[79] C.-O. Almbladh e U. von Barth, "Exact results for the charge and spin densities, exchange-correlation potentials, and density-functional eigenvalues," Phys. Rev. B 31 (1985) 3231-3244.

[80] M. E. Köse, "Theoretical prediction of ionization/oxidation potentials in conjugated polymers," Theo. Chem. Accounts 128 (2010) 157-164.

[81] S. Refaely-Abramson, R. Baer, e L. Kronik, "Fundamental and excitation gaps in molecules of relevance for organic photovoltaics from an optimally tuned range-separated hybrid functional," Phys. Rev. B 84 (2011) 075144.

[82] T. Stein, H. Eisenberg, L. Kronik, e R. Baer, "Fundamental Gaps in Finite Systems from Eigenvalues of a Generalized Kohn-Sham Method," Phys. Rev. Lett. 105 (2010) $4-7$.

[83] S. Kümmel e L. Kronik, "Orbital-dependent density functionals: Theory and applications," Rev. Mod. Phys. 80 (2008) 3-60. 
[84] A. J. Cohen, P. Mori-Sánchez, e W. Yang, "Challenges for density functional theory.," Chem. Rev. 112 (2012) 289-320.

[85] J. P. Perdew, R. G. Parr, M. Levy, e J. L. Balduz, "Density-functional theory for fractional particle number: Derivative discontinuities of the energy," Phys. Rev. Lett. 49 (1982) 1691-1694.

[86] J. P. Perdew e M. Levy, "Comment on "significance of the highest occupied kohn-sham eigenvalue"," Phys. Rev. B 56 (1997) 16021-16028.

[87] N. Marom, F. Caruso, X. Ren, O. T. Hofmann, T. Körzdörfer, J. R. Chelikowsky, A. Rubio, M. Scheffler, e P. Rinke, "Benchmark of gw methods for azabenzenes," Phys. Rev. B 86 (2012) 245127.

[88] X. Blase, C. Attaccalite, e V. Olevano, "First-principles GW calculations for fullerenes, porphyrins, phtalocyanine, and other molecules of interest for organic photovoltaic applications," Phys. Rev. B 83 (2011) 1-9.

[89] S. Braun, W. R. Salaneck, e M. Fahlman, "Energy-Level Alignment at Organic/Metal and Organic/Organic Interfaces," Adv. Mater. 21 (Apr., 2009) 1450-1472.

[90] S. Kang, Y. Yi, C. Kim, S. Cho, M. Noh, K. Jeong, e C. Whang, "Energy level diagrams of C60/pentacene/Au and pentacene/C60/Au," Synth. Met. 156 (2006) $32-37$.

[91] M. Linares, D. Beljonne, J. Cornil, K. Lancaster, J.-L. Breİdas, S. Verlaak, A. Mityashin, P. Heremans, A. Fuchs, C. Lennartz, J. Ideİ, R. Meİreau, P. Aurel, L. Ducasse, e F. Castet, "On the Interface Dipole at the Pentacene/Fullerene Heterojunction: A Theoretical Study," J. Phys. Chem. C 114 (2010) 3215-3224.

[92] R. Giro e M. J. Caldas, "Atomistic molecular dynamics study of interface formation: Al on poly(p-phenylene vinylene)," Phys. Rev. B 78 (2008) 155312.

[93] N. Koch, "Electronic structure of interfaces with conjugated organic materials," physica status solidi (RRL) - Rapid Research Letters 6 (2012), no. 7, 277-293. 
[94] H. Ishii, K. Sugiyama, E. Ito, e K. Seki, "Energy Level Alignment and Interfacial Electronic Structures at Organic/Metal and Organic/Organic Interfaces," Adv. Mater. 11 (1999), no. 8, 605-625.

[95] F. Flores, J. Ortega, e H. Vázquez, "Modelling energy level alignment at organic interfaces and density functional theory.," Phys. Chem. Chem. Phys. 11 (2009), no. $39,8658-75$.

[96] L.-L. Wang e H.-P. Cheng, "Density functional study of the adsorption of a C60 monolayer on $\mathrm{Ag}(111)$ and $\mathrm{Au}(111)$ surfaces," Phys. Rev. B 69 (2004), no. 16, 165417.

[97] H. Li, Y. Duan, V. Coropceanu, e J.-L. Bredas, "Electronic structure of the pentacene-gold interface: A density-functional theory study," Organic Electronics $\mathbf{1 0}$ (2009), no. 8, 1571-1578.

[98] K. Tonigold e A. Gross, "Adsorption of small aromatic molecules on the (111) surfaces of noble metals: A density functional theory study with semiempirical corrections for dispersion effects.," J. Chem. Phys. 132 (2010), no. 22, 224701.

[99] E. Abad, Y. J. Dappe, J. I. Martínez, F. Flores, e J. Ortega, "C6H6/Au(111): interface dipoles, band alignment, charging energy, and van der Waals interaction.," J. Chem. Phys. 134 (2011), no. 4, 044701.

[100] L. Ferrighi, G. K. H. Madsen, e B. r. Hammer, "Self-consistent meta-generalized gradient approximation study of adsorption of aromatic molecules on noble metal surfaces.," J. Chem. Phys. 135 (2011), no. 8, 084704.

[101] P. Medeiros, G. Gueorguiev, e S. Stafström, "Benzene, coronene, and circumcoronene adsorbed on gold, and a gold cluster adsorbed on graphene: Structural and electronic properties," Phys. Rev. B 85 (2012), no. 20, 205423.

[102] S. Dag e L.-w. Wang, "Modeling of nanoscale morphology of regioregular poly(3-hexylthiophene) on a ZnO (1010) surface.," Nano letters 8 (Dec., 2008) 4185-90.

[103] W. Salaneck, Conjugated Polymer and Molecular Interfaces: Science and Technology for Photonic and Optoelectronic Application. Marcel Dekker, 2002. 
[104] P. Haas, F. Tran, e P. Blaha, "Calculation of the lattice constant of solids with semilocal functionals," Phys. Rev. B 79 (2009) 085104.

[105] J. c. v. Klimeš, D. R. Bowler, e A. Michaelides, "Van der waals density functionals applied to solids," Phys. Rev. B 83 (2011) 195131.

[106] N. Ashcroft e N. Mermin, Solid state physics. Science: Physics. Saunders College, 1976.

[107] E. van Lenthe, E. J. Baerends, e J. G. Snijders, "Relativistic total energy using regular approximations," J. Chem. Phys. 101 (1994), no. 11, 9783-9792.

[108] P. Haas, F. Tran, P. Blaha, L. S. Pedroza, A. J. R. da Silva, M. M. Odashima, e K. Capelle, "Systematic investigation of a family of gradient-dependent functionals for solids," Phys. Rev. B 81 (2010) 125136.

[109] L. Schimka, R. Gaudoin, J. Klimeš, M. Marsman, e G. Kresse, "Lattice constants and cohesive energies of alkali, alkaline-earth, and transition metals: Random phase approximation and density functional theory results," Phys. Rev. B 87 (2013), no. $21,214102$.

[110] N. E. Singh-Miller e N. Marzari, "Surface energies, work functions, and surface relaxations of low-index metallic surfaces from first principles," Phys. Rev. B $\mathbf{8 0}$ (2009) 235407.

[111] J. Da Silva, "All-electron first-principles calculations of clean surface properties of low-Miller-index Al surfaces," Phys. Rev. B 71 (2005), no. 19, 195416.

[112] T. Rangel, D. Kecik, P. E. Trevisanutto, G.-M. Rignanese, H. Van Swygenhoven, e V. Olevano, "Band structure of gold from many-body perturbation theory," Phys. Rev. B 86 (2012), no. 12, 125125.

[113] N. V. Smith, G. K. Wertheim, S. Hüfner, e M. M. Traum, "Photoemission spectra and band structures of $d$-band metals. iv. x-ray photoemission spectra and densities of states in Rh, Pd, Ag, Ir, Pt, and Au," Phys. Rev. B 10 (1974) 3197-3206. 
[114] J. Paier, M. Marsman, K. Hummer, G. Kresse, I. C. Gerber, e J. G. Angyán, "Screened hybrid density functionals applied to solids.," J. Chem. Phys. 124 (2006) 154709.

[115] M. Marsman, J. Paier, A. Stroppa, e G. Kresse, "Hybrid functionals applied to extended systems.," Journal of physics. Condensed matter : an Institute of Physics journal 20 (2008) 064201.

[116] Y. Han e D.-J. Liu, "Quantum size effects in metal nanofilms: Comparison of an electron-gas model and density functional theory calculations," Phys. Rev. B $\mathbf{8 0}$ (2009) 155404.

[117] S. V. Faleev, O. N. Mryasov, e T. R. Mattsson, "Quasiparticle self-consistent GW calculation of the work functions of $\mathrm{Al}(111), \mathrm{Al}(100)$, and $\mathrm{Al}(110), "$ Phys. Rev. B 81 (2010), no. 20, 205436.

[118] G. V. Hansson e S. A. Flodström, "Photoemission study of the bulk and surface electronic structure of single crystals of gold," Phys. Rev. B 18 (1978) 1572-1585.

[119] G. Li, I. Tamblyn, V. R. Cooper, H.-J. Gao, e J. B. Neaton, "Molecular adsorption on metal surfaces with van der Waals density functionals," Phys. Rev. B 85 (2012), no. $12,121409$.

[120] P. Sony, P. Puschnig, D. Nabok, e C. Ambrosch-Draxl, "Importance of van der waals interaction for organic molecule-metal junctions: Adsorption of thiophene on $\mathrm{cu}(110)$ as a prototype," Phys. Rev. Lett. 99 (2007) 176401.

[121] H. Yildirim, T. Greber, e A. Kara, "Trends in Adsorption Characteristics of Benzene on Transition Metal Surfaces: Role of Surface Chemistry and van der Waals Interactions," J. Phys. Chem. C 117 (2013), no. 40, 20572-20583.

[122] W. Liu, J. Carrasco, B. Santra, A. Michaelides, M. Scheffler, e A. Tkatchenko, "Benzene adsorbed on metals: Concerted effect of covalency and van der Waals bonding," Phys. Rev. B 86 (2012), no. 24, 245405.

[123] H. Vázquez, F. Flores, R. Oszwaldowski, J. Ortega, R. Pérez, e a. Kahn, "Barrier formation at metal-organic interfaces: dipole formation and the charge neutrality level," Appl. Surf. Sci. 234 (2004), no. 1-4, 107-112. 
[124] G. Rangger, O. Hofmann, L. Romaner, G. Heimel, B. Bröker, R.-P. Blum, R. Johnson, N. Koch, e E. Zojer, "F4TCNQ on Cu, Ag, and Au as prototypical example for a strong organic acceptor on coinage metals," Phys. Rev. B 79 (2009), no. $16,165306$.

[125] J. I. Martínez, E. Abad, F. Flores, e J. Ortega, "Simulating the organic-molecule/metal interface TCNQ/Au(111)," Phys. Status Solidi B 6 (2011), no. $111, \mathrm{n} / \mathrm{a}-\mathrm{n} / \mathrm{a}$.

[126] V. G. Ruiz, W. Liu, E. Zojer, M. Scheffler, e A. Tkatchenko, "Density-Functional Theory with Screened van der Waals Interactions for the Modeling of Hybrid Inorganic-Organic Systems," Phys. Rev. Lett. 108 (2012), no. 14, 146103.

[127] M. Rosa, S. Corni, e R. Di Felice, "A Density Functional Theory Study of Cytosine on $\mathrm{Au}(111), "$ J. Phys. Chem. C 116 (2012), no. 40, 21366-21373.

[128] Y. Morikawa, K. Toyoda, I. Hamada, S. Yanagisawa, e K. Lee, "First-principles theoretical study of organic/metal interfaces: Vacuum level shifts and interface dipoles," Current Applied Physics 12 (2012) S2-S9.

[129] W. Liu, V. G. Ruiz, G.-X. Zhang, B. Santra, X. Ren, M. Scheffler, e A. Tkatchenko, "Structure and energetics of benzene adsorbed on transition-metal surfaces: density-functional theory with van der Waals interactions including collective substrate response," New. J. Phys. 15 (2013), no. 5, 053046.

[130] K. Toyoda, I. Hamada, K. Lee, S. Yanagisawa, e Y. Morikawa, "Density functional theoretical study of pentacene/noble metal interfaces with van der Waals corrections: vacuum level shifts and electronic structures.," J. Chem. Phys. 132 (2010), no. 13, 134703.

[131] B. Pieczyrak, E. Abad, F. Flores, e J. Ortega, "Charging energy and barrier height of pentacene on $\mathrm{Au}(111)$ : a local-orbital hybrid-functional density functional theory approach.," J. Chem. Phys. 135 (2011), no. 8, 084702.

[132] Y. Morikawa, H. Ishii, e K. Seki, "Theoretical study of n-alkane adsorption on metal surfaces," Phys. Rev. B 69 (2004), no. 4, 1-4. 
[133] O. T. Hofmann, V. Atalla, N. Moll, P. Rinke, e M. Scheffler, "Interface dipoles of organic molecules on $\mathrm{Ag}(111)$ in hybrid density-functional theory," New. J. Phys. 15 (2013), no. 12, 123028.

[134] T. S. Chwee e M. B. Sullivan, "Adsorption studies of $\mathrm{C}(6) \mathrm{H}(6)$ on $\mathrm{Cu}(111), \mathrm{Ag}$ (111), and $\mathrm{Au}$ (111) within dispersion corrected density functional theory.," J. Chem. Phys. 137 (2012), no. 13, 134703.

[135] D. Lu, Y. Li, D. Rocca, e G. Galli, "Ab initio calculation of van der waals bonded molecular crystals," Phys. Rev. Lett. 102 (2009) 206411.

[136] R. Colle, G. Grosso, A. Ronzani, e C. M. Zicovich-Wilson, "Structure and x-ray spectrum of crystalline poly(3-hexylthiophene) from dft-van der waals calculations," Phys. Status Solidi B 248 (2011) 1360-1368.

[137] E. R. Johnson, I. D. Mackie, e G. a. DiLabio, "Dispersion interactions in density-functional theory," J. Phys. Org. Chem. 22 (2009) 1127-1135.

[138] Y. Li, D. Lu, H.-v. Nguyen, e G. Galli, "van der Waals Interactions in Molecular Assemblies from First-Principles Calculations," J. Phys. Chem. A 114 (2010) $1944-1952$.

[139] N. Marom, J. Bernstein, J. Garel, A. Tkatchenko, E. Joselevich, L. Kronik, e O. Hod, "Stacking and Registry Effects in Layered Materials: The Case of Hexagonal Boron Nitride," Phys. Rev. Lett. 105 (2010) 1-4.

[140] N. Marom, A. Tkatchenko, M. Scheffler, e L. Kronik, "Describing Both Dispersion Interactions and Electronic Structure Using Density Functional Theory: The Case of Metal-Phthalocyanine Dimers," J. Chem. Theory Comput. 6 (2010) 81-90.

[141] V. Havu, V. Blum, P. Havu, e M. Scheffler, "Efficient o(n) integration for all-electron electronic structure calculation using numeric basis functions," J. Comp. Phys. 228 (2009) $8367-8379$.

[142] A. Willand, M. Gramzow, S. Alireza Ghasemi, L. Genovese, T. Deutsch, K. Reuter, e S. Goedecker, "Structural metastability of endohedral silicon fullerenes," Phys. Rev. B 81 (2010) 1-4. 
[143] X. Ren, P. Rinke, e M. Scheffler, "Exploring the random phase approximation: Application to CO adsorbed on Cu(111)," Phys. Rev. B 80 (2009) 045402. 